\title{
INDOEX aerosol: A comparison and summary of chemical, microphysical, and optical properties observed from land, ship, and aircraft
}

\author{
A. D. Clarke, ${ }^{1}$ S. Howell, ${ }^{1}$ P. K. Quinn, ${ }^{2}$ T. S. Bates,${ }^{2}$ J. A. Ogren, ${ }^{3}$ E. Andrews, ${ }^{3}$ \\ A. Jefferson, ${ }^{3}$ A. Massling, ${ }^{4}$ O. Mayol-Bracero, ${ }^{5}$ H. Maring, ${ }^{6}$ D. Savoie, ${ }^{6}$ and G. Cass ${ }^{7}$ \\ Received 1 March 2001; revised 16 August 2001; accepted 30 August 2001; published 15 October 2002.
}

[1] The Indian Ocean Experiment (INDOEX) measurements on land, sea, and in the air were designed to provide complementary assessment of chemical, physical, and optical properties of the haze aerosol over the Indian Ocean. Differences in platform requirements and objectives resulted in diverse techniques, measurements, and analyses being employed. In order to best interpret the properties of the INDOEX aerosol, comparisons of data by platform, air mass origin, and light scattering intensity were undertaken. These revealed significant variability in platform averages of aerosol extensive properties (e.g., mass, light scattering, and absorption) but less variability in intensive properties (e.g., mass scattering efficiency, single scattering albedo, backscatter fraction, and Ångström exponent) and the ratios of constituents. In general, ratios of chemical species were found to show greater variability than properties of the size distributions or aerosol optical properties. Even so, at higher haze concentrations with higher scattering values, various determinations of the mass scattering efficiency (MSE) at 33\% relative humidity converged on values of about $3.8 \pm 0.3 \mathrm{~m}^{2} \mathrm{~g}^{-1}$, providing a firm constraint upon the description and modeling of haze optical properties. MSE values trended lower with more dilute haze but became more variable in clean air or regions of low concentrations. This cross-platform comparison resolved a number of measurement differences but also revealed that regional characterization from different platforms results in differences linked to variability in time and space. This emphasizes the need to combine such efforts with coordinated satellite and modeling studies able to characterize large-scale regional structure and variability. These comparisons also indicate that "closure" between chemical, microphysical, and optical properties across platforms to better than about $20 \%$ will require significant improvements in techniques, calibration procedures, and comparison efforts. INDEX TERMS: 0305 Atmospheric Composition and Structure: Aerosols and particles (0345, 4801); 0345 Atmospheric Composition and Structure: Pollution-urban and regional (0305); 0394 Atmospheric Composition and Structure: Instruments and techniques; KEYWORDS: INDOEX, data comparison, optical properties, chemistry, microphysics, size distributions

Citation: Clarke, A. D., et al., INDOEX aerosol: A comparison and summary of chemical, microphysical, and optical properties observed from land, ship, and aircraft, J. Geophys. Res., 107(D19), 8033, doi:10.1029/2001JD000572, 2002.

\footnotetext{
${ }^{1}$ Department of Oceanography, University of Hawaii, Honolulu, Hawaii, USA.

${ }^{2}$ Pacific Marine Environmental Laboratory, National Oceanic and Atmospheric Administration, Seattle, Washington, USA.

${ }^{3}$ Climate Monitoring and Diagnostics Laboratory, National Oceanic and Atmospheric Administration, Boulder, Colorado, USA.

${ }^{4}$ Institute for Tropospheric Research, Leipzig, Germany.

${ }^{5}$ Department of Biogeochemistry, Max Planck Institute for Chemistry, Mainz, Germany.

${ }^{6}$ RSMAS/MAC, University of Miami, Miami, Florida, USA.

${ }^{7}$ School of Earth and Atmospheric Sciences, Georgia Tech, Atlanta, Georgia, USA.
}

Copyright 2002 by the American Geophysical Union. 0148-0227/02/2001JD000572

\section{Introduction}

[2] A major goal of the Indian Ocean Experiment (INDOEX) was to identify the dominant aerosol constituents advected over the Indian Ocean and to establish links between their properties and related radiative effects. Our intent was to provide a database of both natural and anthropogenic aerosol species and their contribution to regional aerosol radiative properties and related climate effects. One objective was to reduce uncertainties in aerosol radiative forcing through "closure" experiments wherein several alternate measurements and approaches are employed to establish a property. This "redundancy" provides a means of testing measurements in order to identify the sources and nature of the uncertainties involved. 
INDOEX employed several platforms and diverse instrumentation coordinated to meet these objectives over extended spatial and temporal scales [Ramanathan et al., 2001]. Because radiative effects depend upon relationships between aerosol size, composition and optical properties it was important that different measurements be interpreted consistently on a given platform and between platforms. In spite of broad agreement among many of the observations and data sets from the INDOEX intensive field phase (11 February 1999-25 March 1999), some measurement differences and uncertainties were evident. The intent of this paper is to identify uncertainties and to provide a consensus on the INDOEX aerosol chemistry, optical properties and aerosol size distributions such that other researchers and modelers have a common reference for fundamental INDOEX in-situ observations.

\section{Approach}

\subsection{Strategy}

[3] A logical approach to ensure that instrument performance and measurement techniques were comparable during INDOEX was to provide side-by-side comparison of various platforms during the experiment. These platforms included the long-term site at Kashidhoo Climate Observatory (KCO), the R/V Sagar Kanya (SK) [Jayaraman et al., 2001], the R/V Ronald H. Brown (RB) and the NCAR C-130 aircraft (C-130) [Ramanathan et al., 2001]. Actual opportunities for side-by-side comparisons include one C-130-RB flyby and several KCO flybys with the C130 as well as some periods when the RB sampled near $\mathrm{KCO}$. These were less frequent than hoped due to logistical difficulties and because both instrument operation and environmental conditions were less consistent than expected. Also, in order to compare aircraft, ship and ground-based data, only the low altitude legs from the C130 flights are used. Even so, for a 10-min surface leg, comparison of C-130 data collected at speeds of $110 \mathrm{~ms}^{-1}$ to surface-based data requires data to be averaged over the same air volume sampled by the C-130. For example, if surface winds are about $6 \mathrm{~m} \mathrm{~s}^{-1}$ then ship or ground data corresponding to the $10 \mathrm{~min}$ of $\mathrm{C}-130$ data is about $3 \mathrm{~h}(10$ $\min \times 110 / 6)$. Even though the C-130 flew along the wind axis parallel to the surface platform for a $10 \mathrm{~min}$ sample leg the passage of air past the surface platform for the next $3 \mathrm{~h}$ seldom maintains the same speed or direction. Hence, in the presence of aerosol gradients, the C-130 measurements and surface measurements can be intrinsically different even for a $10 \mathrm{~min}$ sample, the shortest practical C-130 sample leg. Furthermore, even under ideal circumstances the $\mathrm{C}-130$ near-surface legs flown at $35 \mathrm{~m}$ altitudes may not reflect surface values when a near surface gradient exists.

[4] In view of the problematic nature of side-by-side measurements between platforms, a strategy for more extended comparison is employed here. A rapid and sensitive measurement of aerosol changes common to all platforms is the aerosol scattering coefficient, $\left(\sigma_{s p}\right)$. Light scattering measurements $(550 \mathrm{~nm})$ at a constrained $\mathrm{RH}$ near $55 \%$ were used to establish Low $\left(\sigma_{s p}<25 \mathrm{Mm}^{-1}\right)$, Medium $\left(25 \mathrm{Mm}^{-1}<\right.$ $\left.\sigma_{s p}<55 \mathrm{Mm}^{-1}\right)$ and High $\left(\sigma_{s p}>55 \mathrm{Mm}^{-1}\right)$ aerosol regimes. Data were partitioned into characteristic values/properties observed for each of these $\mathrm{L}, \mathrm{M}$, and $\mathrm{H}$ ranges and stratified into identifiable source regions when possible. This approach allowed comparison of similar data for similar plume properties even when platforms were not colocated and increased opportunities for cross platform comparisons of INDOEX aerosol characteristics.

[5] Only near-surface flight legs of the C-130 are included. For the size distributions, this was defined as legs below $100 \mathrm{~m}$, while chemical composition was compared for legs up to $600 \mathrm{~m}$ to increase the number of available samples. In the optics section the level legs below $1000 \mathrm{~m}$ were used to compare C-130 measurements with surface platforms. A Student $t$ test showed that differences in average submicrometer optical properties were insignificant at the $95 \%$ confidence level whether the altitude was 1000 $\mathrm{m}, 600 \mathrm{~m}$ or $35 \mathrm{~m}$.

[6] Although average concentrations and their variation are presented here for each platform for these classifications, the intent is not to focus on these extensive aerosol properties, since they are expected to differ markedly with the sampling time each platform spent exposed to each regime. Rather, the intent is to group such data into these three optically stratified classifications to see if differences exist in intensive properties such as the mass scattering efficiency (MSE), single-scattering albedo $\left(\varpi_{\mathrm{o}}\right)$, Black Carbon (BC) to Total Carbon (TC) ratio, etc. This analysis depends on the assumptions that 1) each platform accumulated a representative range of values for each regime and 2) fundamental aerosol properties and characteristics were similar on all platforms within each of the $\mathrm{L}, \mathrm{M}$ or $\mathrm{H}$ categories. Hence, differences in ratios evident between platforms or measurement stratifications point to differences in techniques or sampling or to invalid assumptions. Consistent trends in ratios among $\mathrm{L}, \mathrm{M}$, or $\mathrm{H}$ regimes for different platforms or measurements could also indicate a change in intensive aerosol properties with increasing pollution concentrations.

\subsection{Relative Humidity}

[7] One issue important to all platforms and this assessment is the role of relative humidity $(\mathrm{RH})$ on water uptake by the aerosol [Tang and Munkelwitz, 1977]. Water uptake depends on whether or not some of the particle mass is insoluble or partially soluble which, in turn, depends on which chemical species are present. Condensed water is a major aerosol constituent with a pronounced impact on particle size, density, refractive index and scattering extinction [Kochenruther et al., 1999]. It is not only important to the goal of linking aerosol radiative forcing to the various aerosol species but also because most aerosol measurement techniques (e.g., size, mass, optical properties) require understanding and accounting for $\mathrm{RH}$ effects.

[8] Hence, both interpretation of measurements at instrument $\mathrm{RH}$ and the extrapolation of measured properties to ambient RH conditions introduce uncertainties that must be accounted for. For example, various impactors used to size segregate aerosol prior to measurement (e.g., nephelometer, filter, or gravimetric mass) will have a size cut that will fluctuate with $\mathrm{RH}$ due to changes in particle size and density. Efforts to control impactor RH on the ground were often successful but were problematic or not practical for the varied conditions aboard aircraft. Also, aerosol sizing 
Table 1. Expected Influence of an Uncertainty in RH on Various Measurements ${ }^{\mathrm{a}}$

\begin{tabular}{|c|c|c|c|c|}
\hline Property & Measurement & Sensitive To & $\begin{array}{l}\text { Effect of RH } \\
\text { Increase }\end{array}$ & Impact on Measurement \\
\hline Gravimetric mass & Mass at $30 \% \mathrm{RH}$ & Weighing $\mathrm{RH}$ & Increase mass & Overestimate mass \\
\hline \multirow{2}{*}{$\begin{array}{l}\text { Analytical mass } \\
\text { less then } 1 \mu \mathrm{m} \text { Dp } \\
\text { (impactor) }\end{array}$} & $\mathrm{OC}$ & Size cut & ? & \multirow[t]{2}{*}{ ? } \\
\hline & $\mathrm{TC}$ & Size cut & $?$ & \\
\hline $\begin{array}{l}\text { FSSP size dist. } \\
\text { on wing }(\mathrm{C}-130)\end{array}$ & $\begin{array}{l}\text { Ambient forward } \\
\text { scatter }\end{array}$ & Ref. Index & Decrease R.I. & Size Decrease \\
\hline On wing (C-130) & & Ref. Index & Decrease Dp & Size Decrease \\
\hline DMA size dist. & Aerosol mobility & Measured RH & Decrease & Size Increase \\
\hline \multirow[t]{2}{*}{ APS size dist. } & \multirow{2}{*}{$\begin{array}{l}\text { Aerodynamic Drag } \\
\text { relative to reference }\end{array}$} & Density & Decrease & Size Decrease \\
\hline & & Size & Increase & Size Increase \\
\hline OPC size dist. & $\begin{array}{l}\text { Optical Scatter } \\
\text { relative to reference }\end{array}$ & Ref. Index & Decrease & Size Decrease \\
\hline Light absorption & Filter transmission & Measured RH & $?$ & Absorption Decrease? \\
\hline
\end{tabular}

${ }^{\mathrm{a}} \mathrm{RH}$ increase assumed.

instruments determine size classes by various methods including diffusion, aerodynamic properties, and optical properties and these often involve assumptions about particle shape, density, and refractive index all of which can change with $\mathrm{RH}$ and often in very different ways. Even gravimetric mass will depend on the RH during weighing. Indications of some of these competing effects on INDOEX measurements are illustrated in Table 1 . Here we indicate the impact on a measurement as a result of an actual $\mathrm{RH}$ value being higher than that assumed to be correct for a measurement. INDOEX planning called for conditioning $\mathrm{RH}$ to target values of $55 \%$ when possible (for impactors, nephelometry, etc.) and filter mass measurements at 33\%. However, for the variable conditions aboard the C-130 (pressure, ambient RH etc.) this was not always possible and such adjustments are often necessary.

[9] Two fundamental aerosol measurements made during INDOEX provide valuable constraints on how these RH adjustments are implemented for optical and sizing measurements. The first is the change in scattering coefficient with humidity, typically called $\mathrm{f}(\mathrm{RH})$. This is the ratio of scattering at a given $\mathrm{RH}$ to that at some reference humidity (chosen here as $40 \% \mathrm{RH})$. The $\mathrm{f}(\mathrm{RH})$ for INDOEX was measured at $\mathrm{KCO}$ by maintaining one nephelometer at $40 \%$ RH while gradually scanning the humidity within a second nephelometer. For the near surface data compared here we assume the dominant dependency found at KCO applies (Figure 1 ) although variability of about $\pm 15 \%$ around this line is present in the full data set (J. A. Ogren, personal communication, 2001). The average relationship shown in Figure 1 has been used to adjust light scattering measured at one RH to light scattering at a different RH when necessary. This approach of scanning a range of $\mathrm{RH}$ used at $\mathrm{KCO}$ was too slow for $\mathrm{f}(\mathrm{RH})$ measurements on the $\mathrm{C}-130$ and a simpler method was used where one nephelometer operated at aircraft temperature while the second was controlled to a target humidity near $85 \%$. This provided a two-point characterization of the plot shown in Figure 1.

[10] The ratio of wet to dry aerosol diameters or the socalled growth factor, D/Do, places another empirical constraint on the effect of soluble/insoluble constituents on particle growth behavior. The associated measurement of
$\mathrm{D} / \mathrm{D}_{\text {ref }}$ (where "ref" here is about $55 \% \mathrm{RH}$ for this data) was measured on the RB using a Tandem Differential Mobility Analyzer (TDMA) technique (A. Massling et al., Hygroscopic properties and solubility of different aerosol types over the Indian Ocean, submitted to Journal of Geophysical Reasearch, 2001) and provides an empirical approach for scaling sizes measured at one RH to sizes at some other RH (Figure 2). Here we show the RB measured data for the largest "dry" particle sizes characterized (250 nm geometric diameter at less than $10 \% \mathrm{RH}$ ) by the TDMA. Since most aerosol optical properties during INDOEX were dominated by accumulation mode aerosol (see below), this is also the most appropriate TDMA size range to use for corrections for particle growth related to their optical properties.

[11] A second plot of $\mathrm{D} / \mathrm{D}_{\text {ref }}$ included in Figure 2 is taken from the ACE2 measurements of Swietliki et al. [2000]. Their data has been "normalized" to agreement with Massling data at $55 \% \mathrm{RH}$. This was done because Swietliki growth was originally referenced to "dry" diameters at a low RH of about $13 \%$ while Massling data was referenced to about 5\%RH for "dry" diameters. Normalizing data sets to $55 \% \mathrm{RH}$ avoids apparent differences caused by uncertain

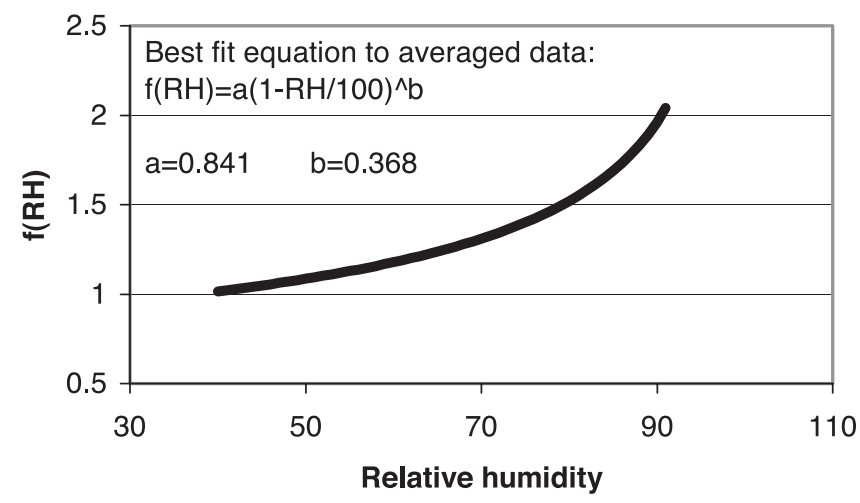

Figure 1. Equation of curve fit to average $\mathrm{f}(\mathrm{RH})$ a function of relative humidity measured by CMDL at KCO for $\mathrm{Dp}<10 \mu \mathrm{m}$. Scatter in original data is about $\pm 15 \%$ around this line (not shown). 


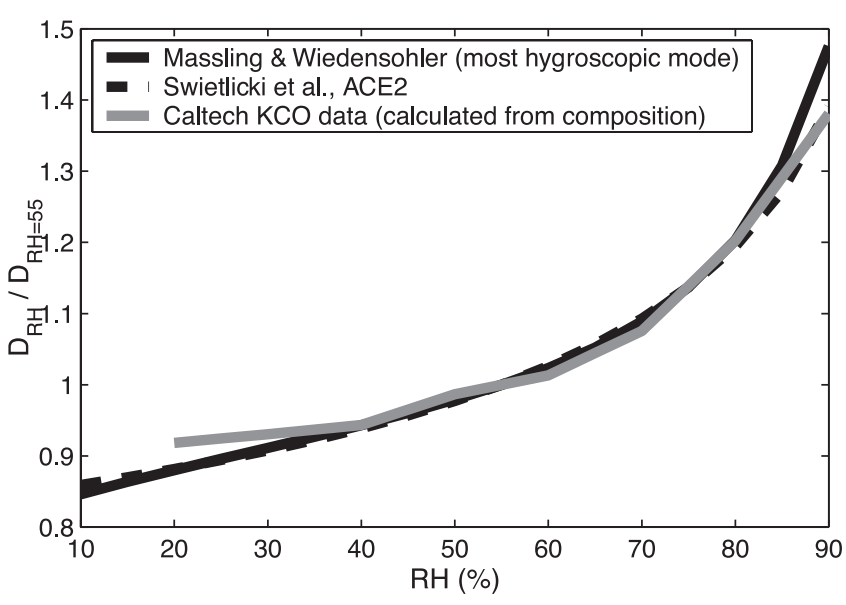

Figure 2. $\mathrm{D} / \mathrm{D}_{\text {ref }}$ a function of $\mathrm{RH}$ measured by DMPS on the RB for both INDOEX (solid line) and ACE-2 (dashed line) experiments and estimated values derived from Caltech KCO chemistry data (see text).

growth behavior below $10 \% \mathrm{RH}$ including the greater uncertainty associated with $\mathrm{RH}$ measurements at low RH. Also shown in Figure 2 is the calculated normalized growth for a limited period of size-resolved chemical data taken at $\mathrm{KCO}$ that shows very similar inferred growth behavior for $\mathrm{RH}$ values above about $35 \% \mathrm{RH}$. The growth equation obtained by fitting these data provides a consistent way to adjust sizes at one RH (above 35\% RH) to sizes expected at another RH.

[12] Specification of $\mathrm{RH}$ is also essential to interpreting aerosol intensive parameters discussed toward the end of this paper, such as the ratio of aerosol scattering to aerosol mass (the mass scattering efficiency or MSE). We will show this important intensive parameter at $\mathrm{RH}=33 \%$. This was the lowest RH at which measurements were routinely made (gravimetric mass on the RB) and is selected here because gravimetric mass change in response to RH was not measured. In order to facilitate linking these data (e.g., MSE) to measurements at other conditions the functional dependencies used to describe $\mathrm{f}(\mathrm{RH})$ and $\mathrm{D} / \mathrm{D}_{\text {ref }}$ have also been included.

[13] We note that while dry MSE might be more useful for incorporating these data into chemical transport models or other applications that use dry mass as a variable, it would require extrapolation of both mass and scattering to $\mathrm{RH}$ conditions well below any measurements we made. Figure 2, which shows discrepancies between measured $\mathrm{D} / \mathrm{D}_{\text {ref }}$ and chemically estimated values at low RH, suggests that such extrapolations are risky. However, if we had pursued such extrapolation, the lower RH Massling TDMA data indicate that $\mathrm{D}(33) / \mathrm{D}(0)$ is 1.14 , which translates into 1.48 times the dry volume or 1.22 times the mass for spherical particles with dry density of $2.2 \mathrm{~g} \mathrm{~cm}^{-3}$. Similarly, the $\mathrm{f}(\mathrm{RH})$ curve fit (Figure 1) suggests that scattering at $33 \% \mathrm{RH}$ exceeds dry scattering by $16 \%$, but no data are available to confirm that extrapolation. Both cases indicate significant water remaining at $33 \%$ compared to the dry state.

\subsection{Comparison of Indoex Data Products}

[14] Measurements can reflect differences in sample platforms, instrument, instrument operation/configuration, sam- pling inlets, environmental conditions, sample periods, locations etc. In preparation for INDOEX, efforts were made to make various measurements as comparable as possible within the constraints common to most field studies. Summaries of the sampling approach for each of the platforms can be found in Appendix A. Additional detailed discussions of the chemical, physical, and optical properties measured and specific issues related to sampling and instrumentation are also presented in Appendix B. We encourage the reader who is concerned with these measurement issues to read these appendices as a reference for the discussions that follow.

\subsection{Chemical Properties}

[15] The aerosol chemical species considered here and measured on the $\mathrm{RB}$, the $\mathrm{C}-130$, and at $\mathrm{KCO}$ are those important to aerosol radiative forcing. These include non sea-salt (nss) $\mathrm{SO}_{4}^{2-}$, black carbon (BC), organic carbon (OC), total carbon (TC) which is the sum of BC and OC, and aerosol mass. Sea-salt is not considered because it was a minor component in the submicrometer size range in the $\mathrm{NH}$ samples and because submicrometer aerosol dominated INDOEX optical properties (see size discussion below). Absolute concentrations of these species are compared as are ratios of the mass concentration of various species, scattering to mass, and absorption to BC. Only submicrometer aerosol (those with an aerodynamic diameter less than or equal to one micrometer) are included in these comparisons.

[16] No side-by-side comparisons between platforms or between $\mathrm{KCO}-\mathrm{UMiami}$ and $\mathrm{KCO}-\mathrm{Caltech}$ were possible due to a lack of data on at least one platform during those time periods. Instead, comparisons of the chemical species (concentrations and ratios) were made based on similarities in sampling conditions for $\mathrm{L}, \mathrm{M}$, and $\mathrm{H}$ scattering categories (Table 2), trajectories to the platforms (from the Bay of Bengal (BoB) or the Arabian Sea (AS)) (Table 3), and, for the RB and C-130, geographical regions (Northern Hemisphere, ITCZ, and Southern Hemisphere) (Table 4). Comparisons for the low scattering category do not include cases where the RB or C-130 experienced trajectories from the northern or southern Indian Oceans in order to make those data more comparable to the KCO data. Figures $3 a-3 c$ summarizes the typical values for major components and properties on each platform and under each $\mathrm{L}, \mathrm{M}$ or $\mathrm{H}$ condition.

\subsubsection{Nss $\mathrm{SO}_{4}^{2-}$}

[17] Mean nonsea-salt sulfate (nss $\mathrm{SO}_{4}^{2-}$ ) concentrations from KCO-Caltech and the RB agreed within $26 \%$ for the low and medium scattering regimes (Table 2; Figure 3a). KCO-UMiami and C-130 mean values were considerably lower and did not agree with each other or with the other platforms within 1 standard deviation. For the high scattering category (Table 2; Figure 3a), the mean RB concentration was higher than the KCO-UMiami and $\mathrm{C}-130$ values by $40 \%$ and higher than the KCO-Caltech values by $20 \%$. However, due to the large variability in concentrations in this category, all differences between platforms were within 1 standard deviation of the means.

[18] Separating the data according to trajectories reveals that the large variability is due in part to air mass flow patterns to the sampling platforms. For trajectories from the AS, the $\mathrm{RB}$ mean nss $\mathrm{SO}_{4}^{2-}$ concentration is lower than the KCO- 
Table 2. Mean Concentrations and Mass Ratios Obtained for KCO, RB, and C-130 Measurements for Submicrometer Chemical Species for Harmony Categories "Low," "Medium, and "High" Scattering ${ }^{a}$

\begin{tabular}{|c|c|c|c|c|}
\hline Species & KCO-UMiami & KCO-Caltech ${ }^{\mathrm{b}}$ & $\mathrm{C}-130$ & $\mathrm{RB}$ \\
\hline $\begin{array}{l}\text { Concentrations } \\
{\mathrm{Nss} \mathrm{SO}_{4}^{2-}}_{\mathrm{BC}} \\
\mathrm{OC} \\
\mathrm{TC} \\
\text { Total mass }\end{array}$ & $\begin{array}{l}\text { N/A } \\
\text { N/A } \\
\text { N/A } \\
\text { N/A }\end{array}$ & $\begin{array}{l}\left(\sigma_{s p} \leq 25 \mathrm{Mm}^{-1}\right) \\
\mu \mathrm{g} \mathrm{m}^{-3} \\
2.3 \pm 0.04 \\
0.55 \pm 0.03(1) \\
0.61 \pm 0.10(1) \\
1.2 \pm 0.11(1) \\
7.9 \pm 0.50\end{array}$ & $\begin{array}{l}\mu \mathrm{g} \mathrm{m}^{-3} \\
0.51 \pm 0.30(3) \\
\text { cnd - } 0.5(3) \\
\text { cnd (3) } \\
\text { cnd (3) } \\
\text { cnd (3) }\end{array}$ & $\begin{array}{l}\mu \mathrm{g} \mathrm{m}^{-3} \\
1.7 \pm 0.40(8) \\
0.17 \pm 0.21(2) \\
0.27 \pm 0.20(2) \\
0.44 \pm 0.01(2) \\
3.9 \pm 0.66(4)\end{array}$ \\
\hline 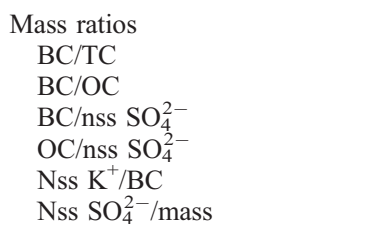 & $\begin{array}{l}\text { N/A } \\
\text { N/A } \\
\text { N/A } \\
\text { N/A } \\
\text { N/A } \\
\text { N/A }\end{array}$ & $\begin{array}{l}0.47 \pm 0.05(1) \\
0.90 \pm 0.14(1) \\
0.24 \pm 0.01(1) \\
0.26 \pm 0.04(1) \\
\text { N/A } \\
0.29 \pm 0.02(1)\end{array}$ & $\begin{array}{l}\text { cnd (3) } \\
\text { cnd (3) } \\
\text { cnd (3) } \\
\text { cnd (3) } \\
\text { cnd (3) } \\
\text { cnd (3) }\end{array}$ & $\begin{array}{l}0.39 \pm 0.47(2) \\
1.4 \pm 1.8(2) \\
0.07 \pm 0.08(2) \\
0.17 \pm 0.17(2) \\
0.56 \pm 0.24(2) \\
0.39 \pm 0.08(3)\end{array}$ \\
\hline $\begin{array}{l}\text { Scattering to mass ratios } \\
\sigma_{s p}(33 \%) / \text { mass }(33 \%)^{\mathrm{c}} \\
\sigma_{s p}(33 \%) / \text { mass }(\text { chem anal })^{\mathrm{d}} \\
\sigma_{a p} / \mathrm{BC}\end{array}$ & $\begin{array}{l}\mathrm{m}^{2} \mathrm{~g}^{-1} \\
\text { N/A } \\
\text { N/A } \\
\text { N/A }\end{array}$ & $\begin{array}{l}\mathrm{m}^{2} \mathrm{~g}^{-1} \\
3.7(1) \\
20(1)\end{array}$ & $\begin{array}{l}\mathrm{m}^{2} \mathrm{~g}^{-1} \\
\text { cnd } \\
12(1)\end{array}$ & $\begin{array}{l}\mathrm{m}^{2} \mathrm{~g}^{-1} \\
4.1 \pm 1.2(7) \\
5.2 \pm 2.5(7) \\
12(1)\end{array}$ \\
\hline $\begin{array}{l}\text { Concentrations } \\
\text { nss } \mathrm{SO}_{4}^{2-} \\
\mathrm{BC} \\
\mathrm{OC} \\
\mathrm{TC} \\
\text { Total mass }\end{array}$ & $\begin{array}{r}\text { "Medium } \\
3.2 \pm 0.58 \text { (4) }\end{array}$ & $\begin{array}{l}\left(25<\sigma_{s p} \leq 55\right. \\
\mu \mathrm{g} \mathrm{m}^{-3} \\
4.5 \pm 0.04(2) \\
1.4 \pm 0.04(2) \\
1.0 \pm 0.11(2) \\
2.4 \pm 0.12(2) \\
14 \pm 0.68(2)\end{array}$ & $\begin{array}{l}\mu \mathrm{g} \mathrm{m}^{-3} \\
2.2 \pm 0.68(4) \\
0.8 \pm 0.7(4) \\
1.3 \pm 1.2(4) \\
2.1 \pm 1.6(4) \\
7.1 \pm 1.3(3)\end{array}$ & $\begin{array}{l}\mu \mathrm{g} \mathrm{m}^{-3} \\
4.5 \pm 0.78(7) \\
0.43 \pm 0.18(6) \\
0.42 \pm 0.06(6) \\
0.85 \pm 0.22(6) \\
8.9 \pm 2.6(8)\end{array}$ \\
\hline $\begin{array}{l}\text { Mass ratios } \\
\mathrm{BC} / \mathrm{TC} \\
\mathrm{BC} / \mathrm{OC} \\
\mathrm{BC} / \mathrm{nss} \mathrm{SO}_{4}^{2-} \\
\mathrm{OC}_{\mathrm{nss} \mathrm{SO}}^{2-} \\
\mathrm{nss} \mathrm{K}^{+} / \mathrm{BC}^{2-} \\
\mathrm{nss} \mathrm{SO}_{4}^{2-} / \text { mass }\end{array}$ & $0.30 \pm 0.05$ & $\begin{array}{l}0.58 \pm 0.03(2) \\
1.4 \pm 0.16(2) \\
0.31 \pm 0.01(2) \\
0.22 \pm 0.02(2) \\
0.32 \pm 0.015(2)\end{array}$ & $\begin{array}{l}0.40 \pm 0.20(4) \\
0.90 \pm 0.60(4) \\
0.36 \pm 0.28 \text { (3) } \\
0.90 \pm 0.80(3) \\
0.30 \pm 0.30(3) \\
0.35 \pm 0.13(3)\end{array}$ & $\begin{array}{l}0.49 \pm 0.10(6) \\
1.0 \pm 0.39(6) \\
0.10 \pm 0.03(6) \\
0.10 \pm 0.02(6) \\
0.74 \pm 0.20(6) \\
0.45 \pm 0.04(6)\end{array}$ \\
\hline $\begin{array}{l}\text { Scattering to mass ratios } \\
\sigma_{s p}(33 \%) / \text { mass }(33 \%)^{\mathrm{c}} \\
\sigma_{s p}(33 \%) / \text { mass }(\text { chem anal })^{\mathrm{d}} \\
\sigma_{a p} / \mathrm{BC}\end{array}$ & $\begin{array}{l}\mathrm{m}^{2} \mathrm{~g}^{-1} \\
2.2 \pm 0.57\end{array}$ & $\begin{array}{l}\mathrm{m}^{2} \mathrm{~g}^{-1} \\
3.4 \pm 0.09(2) \\
13(1)\end{array}$ & $\begin{array}{l}\mathrm{m}^{2} \mathrm{~g}^{-1} \\
4.7 \pm 1.0 \\
13 \pm 8.9(4)\end{array}$ & $\begin{array}{l}\mathrm{m}^{2} \mathrm{~g}^{-1} \\
3.9 \pm 0.51(6) \\
4.7 \pm 0.36(6) \\
17 \pm 4.6(6)\end{array}$ \\
\hline $\begin{array}{l}\text { Concentrations } \\
\text { nss } \mathrm{SO}_{4}^{2-} \\
\mathrm{BC} \\
\mathrm{OC} \\
\text { TC } \\
\text { Total mass }\end{array}$ & $\begin{array}{r}\text { "Hig } \\
6.4 \pm 1.1 \text { (12) }\end{array}$ & $\begin{array}{l}g\left(55 \mathrm{Mm}^{-1}<\sigma_{s p}\right. \\
\mu \mathrm{g} \mathrm{m}^{-3} \\
7.4 \pm 0.09 \text { (3) } \\
2.5 \pm 0.07(3) \\
2.1 \pm 0.12(3) \\
4.6 \pm 0.14(3) \\
21 \pm 0.49(3)\end{array}$ & $\begin{array}{l}\mu \mathrm{g} \mathrm{m}^{-3} \\
6.2 \pm 2.7(12) \\
2.5 \pm 1.8(12) \\
3.1 \pm 2.2(11) \\
5.5 \pm 3.3(11) \\
14 \pm 6.2(9)\end{array}$ & $\begin{array}{l}\mu \mathrm{g} \mathrm{m}^{-3} \\
8.9 \pm 3.9(14) \\
1.4 \pm 0.48(8) \\
0.95 \pm 0.44(8) \\
2.3 \pm 0.66(8) \\
17 \pm 3.5(3)\end{array}$ \\
\hline 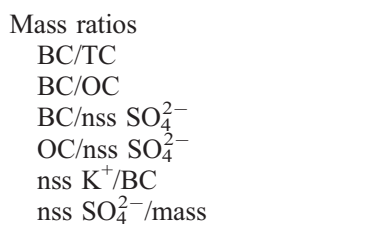 & $0.30 \pm 0.02(12)$ & $\begin{array}{l}0.55 \pm 0.02(3) \\
1.2 \pm 0.07(3) \\
0.34 \pm 0.01(3) \\
0.28 \pm 0.02(3)\end{array}$ & $\begin{array}{l}0.50 \pm 0.10(11) \\
1.1 \pm 0.60(11) \\
0.46 \pm 0.18(13) \\
0.50 \pm 0.30(9) \\
0.11 \pm 0.03(9) \\
0.41 \pm 0.09(10)\end{array}$ & $\begin{array}{l}0.59 \pm 0.12(8) \\
1.6 \pm 0.64(8) \\
0.16 \pm 0.04(8) \\
0.13 \pm 0.11(8) \\
0.37 \pm 0.11(8) \\
0.52 \pm 0.11(7)\end{array}$ \\
\hline $\begin{array}{l}\text { Scattering to mass ratios } \\
\sigma_{s p}(33 \%) / \text { mass }(33 \%)^{\mathrm{c}} \\
\sigma_{s p}(33 \%) / \text { mass }(\text { chem anal })^{\mathrm{d}} \\
\sigma_{a p} / \mathrm{BC}\end{array}$ & $\mathrm{m}_{2} \mathrm{~g}^{-1}$ & $\begin{array}{l}\mathrm{m}^{2} \mathrm{~g}^{-1} \\
3.5 \pm 0.16\end{array}$ & $\begin{array}{l}5.8 \pm 2.0(9) \\
9.2 \pm 2.8(12)\end{array}$ & $\begin{array}{l}\mathrm{m}^{2} \mathrm{~g}^{-1} \\
4.1 \pm 0.23 \\
4.4 \pm 0.08 \\
10 \pm 2(3)\end{array}$ \\
\hline
\end{tabular}

${ }^{a}$ N/A, no sample available; cnd, could not determine. Also shown are \pm 1 standard deviations. Number of samples collected are shown in parentheses. ${ }^{\mathrm{b}}$ Mean and confidence interval.

${ }^{\mathrm{c}}$ Based on scattering coefficients adjusted to $33 \% \mathrm{RH}$ and mass measured gravimetrically at 33\% RH (RB) or 39\% RH (KCO-Caltech).

${ }^{\mathrm{d}}$ Based on scattering coefficients adjusted to $33 \% \mathrm{RH}$ and the sum of the chemically analyzed mass. 
Table 3. Mean Concentrations and Mass Ratios for Submicrometer Chemical Species for all Trajectories Originating From the Bay of Bengal and the Arabian $\mathrm{Sea}^{\mathrm{a}}$

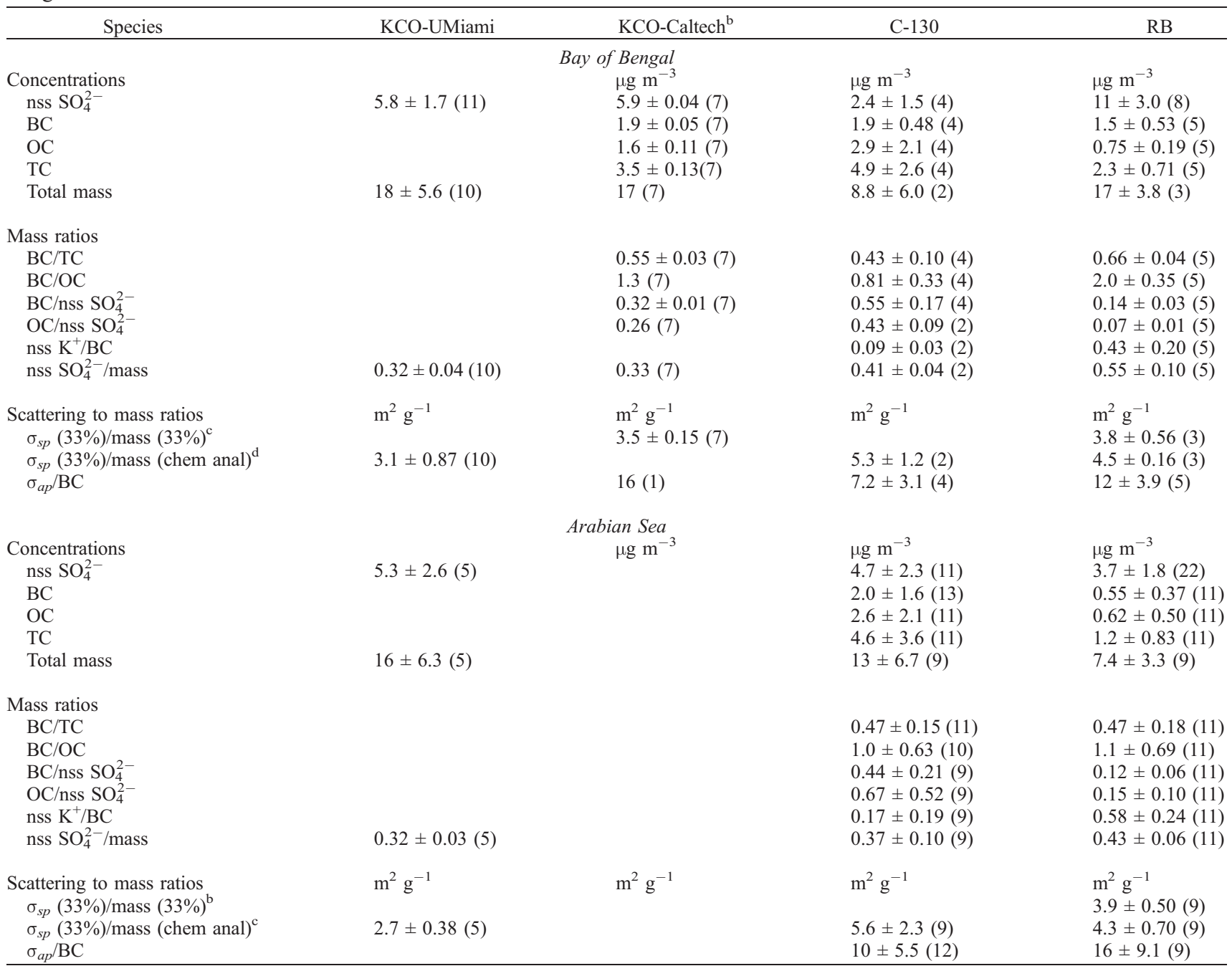

${ }^{a}$ Also shown are \pm 1 standard deviations. Number of samples collected shown in parentheses.

${ }^{\mathrm{b}}$ Mean and confidence interval.

${ }^{\mathrm{c}}$ Based on scattering coefficients adjusted to 33\% RH and mass measured gravimetrically at 33\% RH (RB) or 39\% RH (KCO-Caltech).

${ }^{\mathrm{d}}$ Based on scattering coefficients adjusted to $33 \% \mathrm{RH}$ and the sum of the chemically analyzed mass.

UMiami and the C-130 mean concentrations (Table 3). In contrast, for trajectories from the $\mathrm{BoB}$, the $\mathrm{RB}$ mean concentration is higher than the KCO-UMiami and KCO-Caltech values by almost a factor of two and higher than the $\mathrm{C}$ 130 mean value by over a factor of 4 (Table 3). Sampling schedules of the various platforms may have contributed to these differences. BoB trajectories were sampled by $\mathrm{KCO}$ and the C-130 between 12 February and 3 March and by the RB between 4 March and 5 March and again on 29 March. Even within the BoB trajectories sampled by one platform, however, there is considerable variability in the nss $\mathrm{SO}_{4}^{2-}$ concentrations. The BoB category included trajectories from Calcutta, over southern India or Sri Lanka to the Arabian Sea as well as trajectories from central India to the Arabian Sea. Non-sea salt $\mathrm{SO}_{4}^{2-}$ concentrations measured on the RB were up to a factor of three higher for trajectories from Calcutta compared to trajectories from central India (Figure 3a).

[19] The differences evident in data sorted by scattering regime or trajectory are averaged out when RB and C-130 samples are sorted by geographical region. The mean nss $\mathrm{SO}_{4}^{2-}$ concentration for Northern Hemisphere samples collected on the $\mathrm{RB}$ is $25 \%$ higher than the $\mathrm{C}-130$ Northern Hemisphere mean (Table 4) but lies within 1 standard deviation of both mean concentrations. For all cases, whether sorted by scattering regime, trajectory, or geographical region, the mean $\mathrm{RB}$ nss $\mathrm{SO}_{4}^{2-}$ to mass ratio is about 34\% higher than mean values for the other platforms. $\mathrm{RB}$ mean values ranged from 0.39 to 0.52 while $\mathrm{KCO}$ and $\mathrm{C}-130$ mean values ranged from 0.29 to 0.41 . Only for the RB-C-130 comparison is the difference within 1 standard deviation of the mean concentrations. In addition, $\mathrm{BC}$ to nss $\mathrm{SO}_{4}$ mean ratios are about a factor of two lower for the $\mathrm{RB}$ samples than for the KCO and C-130 samples. The lower $\mathrm{RB}$ ratios relative to the $\mathrm{C}-130$ and $\mathrm{KCO}$ are a result of lower $\mathrm{BC}$ concentrations and intermittently higher nss $\mathrm{SO}_{4}^{2-}$ concentrations. At the same time, however, dry scattering to mass ratios for $\mathrm{KCO}-\mathrm{Caltech}$ and the $\mathrm{RB}$ are consistent across scattering and trajectory categories which suggests that the difference in nss $\mathrm{SO}_{4}^{2-}$ concentrations is a result of 
Table 4. Mean Concentrations and Mass Ratios for Submicrometer Chemical Species as a Function of Latitude ${ }^{\mathrm{a}}$

\begin{tabular}{|c|c|c|c|c|c|c|}
\hline Species & $\mathrm{RB}(\mathrm{NH})$ & $\mathrm{C}-130(\mathrm{NH})$ & RB (ITCZ) & C-130 (ITCZ) & $\mathrm{RB}(\mathrm{SH})$ & $\mathrm{C}-130(\mathrm{SH})$ \\
\hline \multicolumn{7}{|l|}{ Concentrations } \\
\hline nss $\mathrm{SO}_{4}^{2-}$ & $6.0 \pm 4.1(29)$ & $4.8 \pm 1.9(12)$ & $1.6(1)$ & $1.9(2)$ & $0.75 \pm 0.44(20)$ & $0.19(1)$ \\
\hline $\mathrm{BC}$ & $0.86 \pm 0.62(17)$ & $2.1 \pm 1.4(15)$ & & cnd $-1.5(2)$ & $0.05 \pm 0.06$ & cnd \\
\hline $\mathrm{OC}$ & $0.66 \pm 0.43(17)$ & $2.7 \pm 2.1(14)$ & & cnd $-1.2(2)$ & $0.05 \pm 0.03$ & cnd \\
\hline $\mathrm{TC}$ & $1.5 \pm 0.94(17)$ & $4.8 \pm 3.4(14)$ & & cnd $-2.7(2)$ & $0.10 \pm 0.09$ & cnd \\
\hline Total mass & $12 \pm 5.8(17)$ & $13 \pm 6.3$ & $3.5(1)$ & $7.7(1)$ & $1.7 \pm 1.0(11)$ & cnd \\
\hline \multicolumn{7}{|l|}{ Mass ratios } \\
\hline $\mathrm{BC} / \mathrm{TC}$ & $0.53 \pm 0.17(17)$ & $0.50 \pm 0.10$ & & $0.55(1)$ & $0.31 \pm 0.35$ & cnd \\
\hline $\mathrm{BC} / \mathrm{OC}$ & $1.4 \pm 0.74$ & $1.0 \pm 0.6(14)$ & & $1.2(1)$ & $0.80 \pm 0.93$ & cnd \\
\hline $\mathrm{BC} / \mathrm{nss} \mathrm{SO}_{4}^{2-}$ & $0.12 \pm 0.06(17)$ & $0.5 \pm 0.2(11)$ & & & & \\
\hline $\mathrm{OC} / \mathrm{nss} \mathrm{SO}_{4}^{2-}$ & $0.12 \pm 0.10(17)$ & $0.60 \pm 0.50$ & & $0.4(1)$ & $0.07 \pm 0.06$ & cnd \\
\hline $\mathrm{nss} \mathrm{K}^{+} / \mathrm{BC}$ & $0.53 \pm 0.24(17)$ & $0.2 \pm 0.2(11)$ & & $0.12(1)$ & $0.18 \pm 0.21(4)$ & cnd \\
\hline nss $\mathrm{SO}_{4}^{2-} /$ mass & $0.49 \pm 0.13(17)$ & $0.40 \pm 0.10$ & & $0.40(1)$ & $0.50 \pm 0.25$ & cnd \\
\hline \multicolumn{7}{|l|}{ Scattering to mass ratios } \\
\hline$\sigma_{s p}(33 \%) / \operatorname{mass}(33 \%)^{\mathrm{b}}$ & $3.8 \pm 0.49(12)$ & & & & $4.7 \pm 1.4(5)$ & cnd \\
\hline$\sigma_{s p}(33 \%) /$ mass (chem anal $)^{\mathrm{c}}$ & $4.3 \pm 0.60(12)$ & $5.5 \pm 2.0(8)$ & & $8.0(1)$ & $5.4 \pm 1.5(3)$ & \\
\hline$\sigma_{a p} / \mathrm{BC}$ & $13 \pm 4.7(16)$ & $9.5 \pm 5.3(15)$ & & $13(1)$ & $16 \pm 3.4(2)$ & cnd \\
\hline
\end{tabular}

${ }^{\mathrm{a}}$ Here, cnd, could not determine. Northern hemisphere $(\mathrm{NH})$ includes all latitudes north of $1^{\circ} \mathrm{S}$, ITCZ includes all latitudes between $1^{\circ} \mathrm{S}$ and $5^{\circ} \mathrm{S}$, and Southern Hemisphere (SH) includes all latitudes south of $5^{\circ} \mathrm{S}$. Also shown are \pm 1 standard deviations. Number of samples collected shown in parentheses.

${ }^{b}$ Based on scattering coefficients adjusted to $33 \% \mathrm{RH}$ and mass measured gravimetrically at $33 \% \mathrm{RH}$ (RB) or $39 \% \mathrm{RH}$ (KCO-Caltech).

${ }^{\mathrm{c}}$ Based on scattering coefficients adjusted to $33 \% \mathrm{RH}$ and the sum of the chemically analyzed mass.

natural variability in the sampled air masses rather than an analytical effect, i.e., scattering per unit mass of aerosol was consistent but the chemical composition of the aerosol appears to have differed.

\subsubsection{Black Carbon}

[20] For all scattering regimes, mean $\mathrm{BC}$ concentrations were higher for $\mathrm{KCO}$ and the $\mathrm{C}-130$ than for the RB (Tables 2 and 3; Figure 3a). Due to the high variability in the C-130 concentrations, however, differences between the RB and $\mathrm{C}$ 130 were within 1 standard deviation of the C-130 means. Mean concentrations were comparable for $\mathrm{KCO}$ and the $\mathrm{C}$ 130 during Bay of Bengal (BoB) trajectories (Table 3) with the $\mathrm{RB}$ concentrations averaging $30 \%$ lower. In addition, variability in the $\mathrm{BC}$ concentration within the $\mathrm{BoB}$ category was not as great as it was for nss $\mathrm{SO}_{4}^{2-}$ (Figure 3a), at least for the RB,. During AS trajectories, the C-130 mean OC concentration was almost four times greater than the $\mathrm{RB}$ mean concentration (Table 3). Due primarily to the AS difference, the C-130 Northern Hemisphere mean BC concentration was a factor of 2.4 larger than the RB mean Northern Hemisphere concentration. These differences (AS and $\mathrm{NH}$ ) are within 1 standard deviation of the C-130 means.

[21] As for nss $\mathrm{SO}_{4}^{2-}$, the platform differences in the AS category may have been a result of the C-130 and $\mathrm{RB}$ sampling schedules. The highest $\mathrm{BC}$ concentrations were measured by the C-130 between 18 February and 9 March and the RB did not sample AS trajectories until after 7 March. In addition, the estimated concentration of $\mathrm{BC}$ is dependent on the method used to determine the OC/BC split from the measured total carbon. A difference in analytical approaches could have contributed to the observed differences in the $\mathrm{RB}, \mathrm{KCO}$, and $\mathrm{C}-130$ concentrations. Such analytical effects are not indicated by the $\mathrm{BC}$ to $\mathrm{TC}$ and absorption to $\mathrm{BC}$ ratios, however.

[22] $\mathrm{BC} / \mathrm{TC}$ ratios for all platforms were, for the most part, consistent across scattering and trajectory categories with differences within 1 standard deviation of the means (Tables 2 and 3; Figure 3b). An exception was the BoB trajectory category during which the RB mean was 20 and $50 \%$ higher than the $\mathrm{KCO}-\mathrm{Caltech}$ and $\mathrm{C}-130$ mean values, respectively. Overall, C-130 mean values ranged from 0.40 to 0.52 , KCO-Caltech values from 0.47 to 0.58 , and $\mathrm{RB}$ values from 0.39 to 0.66 . The mean Northern Hemisphere ratios were $0.53 \pm 0.17$ and $0.50 \pm 1.0$ on the RB and C-130, respectively.

[23] Mean absorption to $\mathrm{BC}$ ratios on the $\mathrm{C}-130$ and $\mathrm{RB}$ were within 1 standard deviation of the mean values (Tables 2 and 3; Figure 3c) for all scattering and trajectory categories. $\mathrm{RB}$ mean values ranged from 10 to $17 \mathrm{~m}^{2} \mathrm{~g} \mathrm{C}^{-1}$ with a Northern Hemisphere value of $13 \pm 4.7 \mathrm{~m}^{2} \mathrm{~g} \mathrm{C}^{-1}$ (Table 4). $\mathrm{C}-130$ mean values ranged from 7 to $13 \mathrm{~m}^{2} \mathrm{~g} \mathrm{C}^{-1}$ with a Northern Hemisphere mean value of $9.5 \pm 5.3 \mathrm{~m}^{2} \mathrm{~g} \mathrm{C}^{-1}$. KCO-Caltech mean values were comparable to or higher than those measured on the C-130 and RB.

[24] Non-sea salt $\mathrm{K}^{+}$to $\mathrm{BC}$ ratios were compared because aerosol nss $\mathrm{K}^{+}$is a by-product of biomass burning and has been found to correlate well with $\mathrm{BC}$ concentrations in biomass burning plumes [Cachier et al., 1995]. For example, a mean ratio of $0.52 \pm 0.11$ has been reported for biomass burning aerosol in Brazil [Ferek et al., 1998]. Mean ratios ranged from 0.09 to 0.30 on the $\mathrm{C}-130$ with a mean Northern Hemisphere value of $0.2 \pm 0.2$ (Tables 2, 3, and 4; Figure $3 b$ ). Mean ratios were higher on the $\mathrm{RB}$ ranging from 0.37 to 0.74 with a Northern Hemisphere mean of $0.53 \pm$ 0.24 . Differences between the $\mathrm{C}-130$ and $\mathrm{RB}$ were not within 1 standard deviation of the means. Hence, RB measurements suggest an influence of biomass burning emissions on aerosol chemical composition over the Indian Ocean that was not observed to the same extent by the C130.

\subsubsection{Organic Carbon}

[25] For all scattering and trajectory categories mean OC concentrations from the $\mathrm{C}-130$ were a factor of 3 to 4 higher than those from the RB and a factor of 1.3 to 1.8 higher than those from KCO-Caltech (Tables 2 and 3; Figure 3a). However, because of large variability in the $\mathrm{C}-130$ values the differences between the $\mathrm{C}-130$ and $\mathrm{KCO}-\mathrm{Caltech}$ values and the $\mathrm{C}-130$ and $\mathrm{RB}$ values are within 1 standard deviation of the C-130 mean values. Differences between KCOCaltech and the RB are not within 1 standard deviation of the means. In addition to the natural variability discussed above, interplatform differences are most likely a result of 

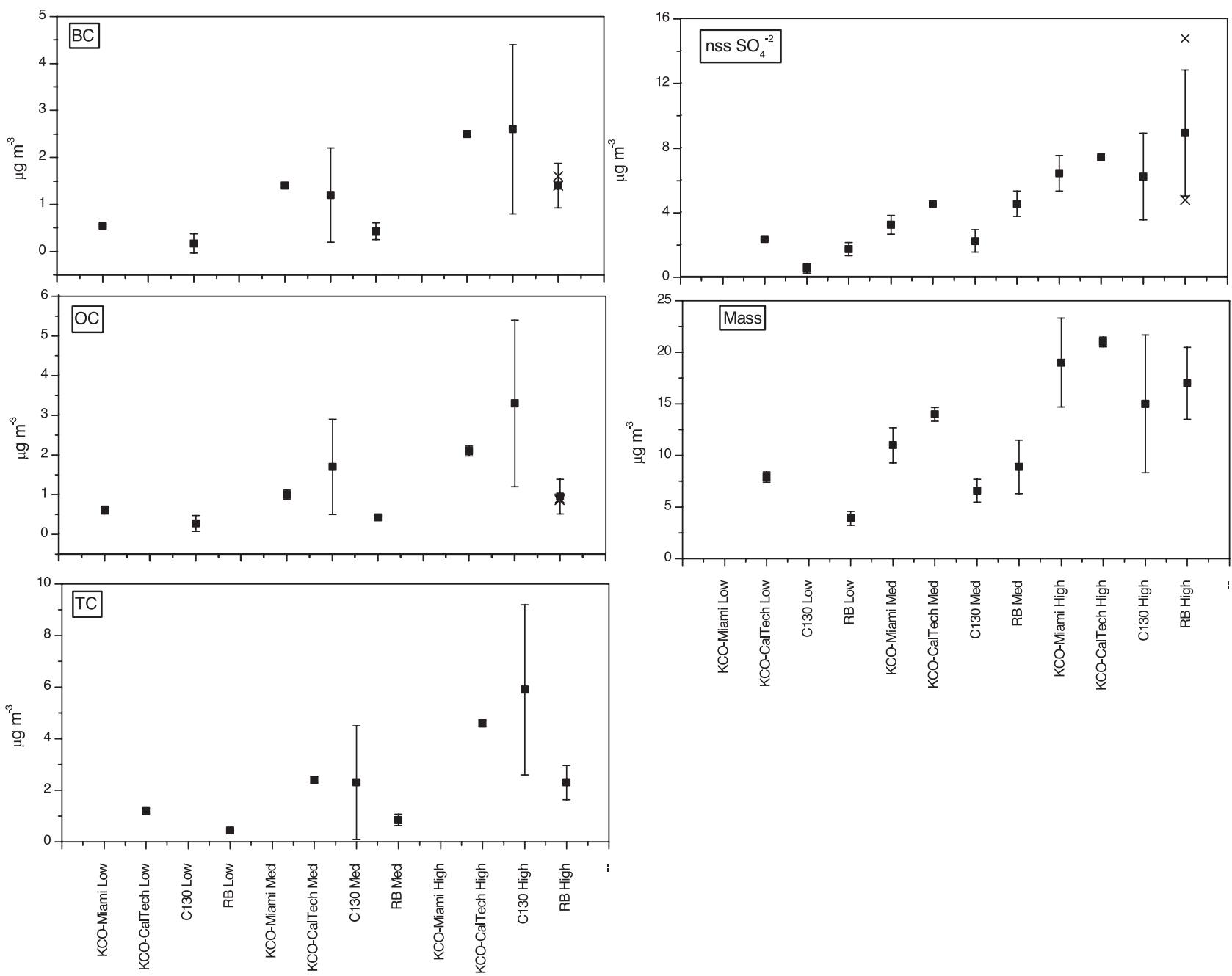

Figure 3a. Means and 1 standard deviation of chemical species measured on the various platforms for the low, medium, and high scattering regimes. Also shown as crosses for RB High scattering regime are the minimum and maximum concentrations measured on the RB during BoB trajectories.

artifacts associated with sampling semivolatile species [Turpin et al., 2000].

[26] $\mathrm{KCO}-\mathrm{Caltech} \mathrm{OC}$ to $\mathrm{nss} \mathrm{SO}_{4}^{2-}$ ratios were relatively constant for all scattering and trajectory categories with mean values ranging from 0.22 to 0.28 (Tables 2 and 3; Figure $3 b)$. For all categories except the BoB trajectory case, RB ratios were lower and ranged from 0.10 to 0.17 . A low mean value of $0.07 \pm 0.01$ was measured in air masses coming from the $\mathrm{BoB}$ and is a function of high nss $\mathrm{SO}_{4}^{2-}$ concentrations. The lower ratios observed on the $\mathrm{RB}$ are due to lower OC and/or higher nss $\mathrm{SO}_{4}^{2-}$ concentrations. C-130 mean ratios were more variable and higher than both the $\mathrm{KCO}-\mathrm{Caltech}$ and $\mathrm{RB}$ values ranging from 0.43 to 0.90 . These relatively high ratios are a result of both higher OC and lower nss $\mathrm{SO}_{4}^{2-}$ concentrations. Mean Northern Hemisphere values were $0.12 \pm 0.10$ and $0.60 \pm 0.50$ for the RB and $\mathrm{C}-130$, respectively (Table 4).

[27] Mean BC to OC ratios ranged from 0.9 to 1.4 over all scattering and trajectory categories at KCO (Caltech), from 0.81 to 1.1 on the $\mathrm{C}-130$, and from 1.0 to 2.0 on the RB. Platform differences between the mean values for all categories are within 1 standard deviation except for the instances where trajectories were from the BoB. Though measured on different days, for this case, the mean C-130 ratio was the lowest observed $(0.81 \pm 0.33)$ and the mean $\mathrm{RB}$ ratio was the highest observed $(2.0 \pm 0.35)$.

\subsubsection{Aerosol Mass}

[28] For the medium and high scattering regimes, submicrometer aerosol mass measured on the C-130 and RB agreed within 1 standard deviation of the mean concentrations (Table 2; Figure 3a). For all scattering regimes where there were data, KCO-UMiami and KCO-Caltech mass concentrations were higher than the $\mathrm{C}-130$ and $\mathrm{RB}$ values and were not within 1 standard deviation of the means. In addition to natural variability, differences may have resulted from the use of nonstandardized methods for collection and analysis. The impactor stages used to determine the KCO-Caltech mass concentrations at ambient RH collected a larger size fraction of the aerosol than did the other samplers (see Appendix B, Table A1). The Sierra impactor used to determine the KCO-UMiami concentrations has a broader submicrometer size cut than the Bernertype impactor used on the RB or the MOUDI used by KCOCaltech. Both of these factors (collection of a larger size 

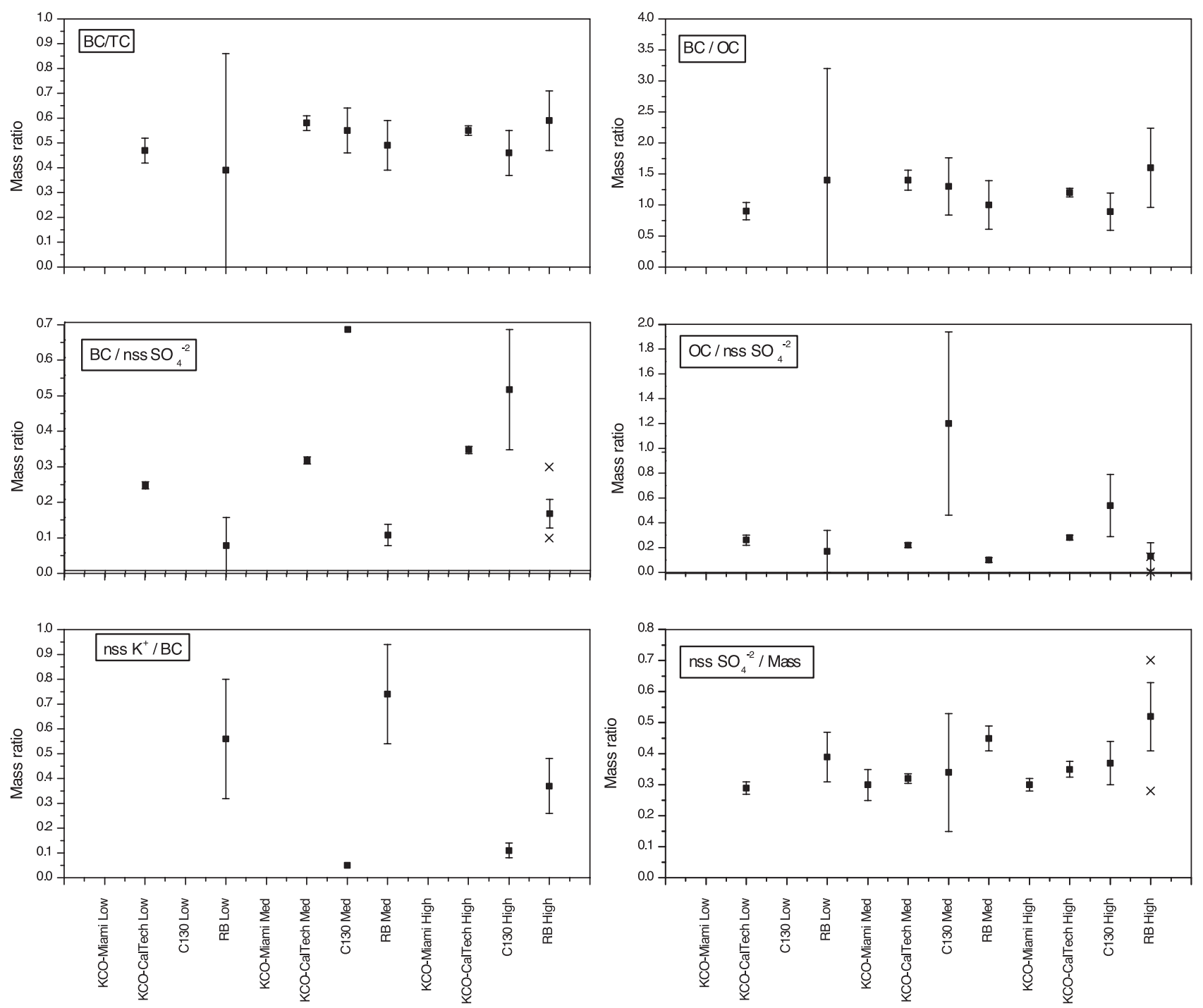

Figure 3b. Means and 1 standard deviation of mass ratios of the chemical species measured on the various platforms for the low, medium, and high scattering regimes. Also shown as crosses for the RB High scattering regime are the minimum and maximum ratios measured on the RB during BoB trajectories.

fraction of the aerosol and a broad size cut) would result in a larger mass concentration. Although the C-130 mass concentrations agreed with the RB values within 1 standard deviation of the means, they were lower than those determined on all other platforms. The $\mathrm{C}-130$ technique of summing the mass of the chemically analyzed species (and excluding water) rather than determining the mass gravimetrically most likely contributed to this difference. In addition, the stacked filter units used on the C-130 had a broad size cut and wider variations in $\mathrm{RH}$ than the other platforms. As discussed below, however, interplatform consistency in scattering to mass ratios suggests that these analytical differences were not great.

\subsubsection{Scattering to Mass Ratios}

[29] Scattering to mass ratios were calculated from scattering coefficients measured at $550 \mathrm{~nm}$ (STP- and angular-corrected as per Anderson and Ogren [1998]) and simultaneously measured gravimetric mass concentrations. The reported ratios (Tables 2, 3, and 4; Figure 3c) include an adjustment of the scattering coefficient to $33 \%$
$\mathrm{RH}$. The adjustment was made by extrapolating the $\mathrm{f}(\mathrm{RH})$ curves measured at KCO (Figure 1) to $33 \%$ RH. For KCO, the KCO-CMDL scattering coefficients were used. RB mass concentrations determined by gravimetric analysis include the amount of water associated with the aerosol on a filter at $33 \pm 3 \%$ RH. KCO-Caltech mass concentrations, which were also determined by gravimetric analysis, include the amount of water associated with the aerosol on a filter at $39 \pm 3 \% \mathrm{RH}$. The KCO-Caltech mass concentrations are not adjusted for change in water mass between $39 \%$ and 33\%RH. Judging from the diameter change in Figure 2 and density calculated from composition, the decrease in mass would be about $3.5 \%$, trivial compared with other sources of disagreement. Chemical mass on the C-130 does not include water or other nonanalyzed species [Mayol-Bracero et al., 2002].

[30] KCO-Caltech and RB MSE ratios agreed within 8 to $17 \%$ for the categories where both platforms had scattering and mass measurements (low, medium, and high scattering, and BoB trajectories) (Tables 2 and 3; Figure 3c). The use of samplers with sharp size cuts and gravimetric analysis to 

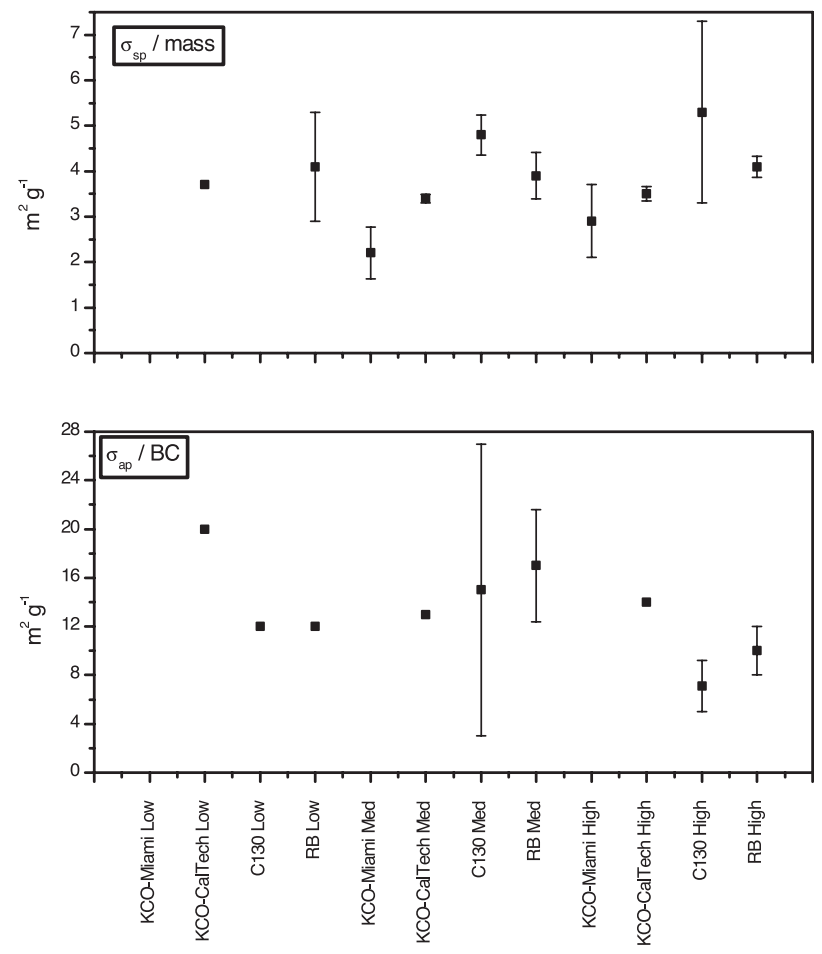

Figure 3c. Means and 1 standard deviation of the scattering to mass and absorption to $\mathrm{BC}$ ratios measured on the various platforms for the low, medium, and high scattering regimes.

derive aerosol mass concentrations most likely contributed to the high level of agreement between these two platforms. KCO-UMiami values agreed with those determined by KCOCaltech within $20 \%$ for the high scattering and BoB trajectory categories. For the four categories that both the RB and C-130 had scattering and mass data (medium and high scattering regime, BoB and AS trajectories), the chemically based mean $\mathrm{C}-130$ scattering to mass ratios were about 20 to $40 \%$ higher than the gravimetric RB means. For the one category that both $\mathrm{KCO}-\mathrm{Caltech}$ and the $\mathrm{C}-130$ had scattering and mass data (high scattering regime), the mean $\mathrm{C}-130$ ratio was about $70 \%$ higher than the KCO-Caltech mean. The consistently higher $\mathrm{C}-130$ values are expected in part due to the derivation of aerosol mass from a summation of the chemical species with no allowance for associated water (see reevaluation below). If the $\mathrm{RB}$ scattering to mass ratios are calculated using the sum of the chemically analyzed mass (Tables 2, 3, and 4), then the $\mathrm{C}-130$ ratios are 18 to $30 \%$ higher (instead of $20-40 \%$ higher).

\subsection{Microphysical Properties}

[31] Before adjusting size distributions (e.g., for 55\% RH) to yield equivalent geometric sizes, as mentioned above, initial checks are made on general instrument performance. When two or more instruments are combined to establish a distribution reasonable agreement must exist in the overlap region for the respective measurements. Because most INDOEX aerosol are well aged there was a negligible fraction of aerosol number at sizes below $20 \mathrm{~nm}$ such that condensation particle counter (CPC) number concentrations encompass virtually all aerosol number and diffusion losses should also be small. Once appropriate size distributions are established then integral number concentrations from the DMPS, DMA-APS, DMA-OPC etc. instrumentation can be compared to total number concentrations obtained from CPC's operated on respective platforms. Assuming that instruments are in optical alignment, the uncertainty in size distribution concentrations from single particle counters is generally limited by flow uncertainties (often about $\pm 1-$ $5 \%$ ). Since the number concentrations for INDOEX are dominated by accumulation mode particles, inlet and transmission losses should be no more than a few percent, constraining the likely differences between size integral number and measured $\mathrm{CN}$ to less than $10 \%$.

[32] We start here with the only side-by-side comparison of the C-130 and RB platforms carried out about 0830 GMT on 28 February 1999. These data were obtained on a $15 \mathrm{~min}$ near surface C-130 flight along the wind and nearby the ship. This corresponds to the air mass passing the RB over about a $3 \mathrm{~h}$ period. Initial excellent agreement between $\mathrm{CN}$ data aboard the $\mathrm{C}-130$ and $\mathrm{RB}$ of about $1,200 \mathrm{~cm}^{-3}$ did not persist for more than an hour after the C-130 left the area. This is believed to be due to a shift in wind direction at the $\mathrm{RB}$ that brought an air mass over the ship that was not sampled by the $\mathrm{C}-130$. Hence, the RB data shown here are for the earlier part of the $3 \mathrm{~h}$ period.

[33] The key size-resolved characteristics for the RB flyby are shown in Figure 4 and reveal the significance of the various moments of the size distribution and their links to aerosol properties. All four panels employ the linear $\mathrm{dZ} /$ $\mathrm{d} \log \mathrm{Dp}$ format such the area under each curve is proportional to the parameter $Z$. Here $Z$ is either number $(N)$, area $(A)$, volume $(\mathrm{V})$ or light scattering $(\sigma)$ and the integral value for each parameter is indicated in the plot. Here we use a vertical shaded bar near $0.75 \mu \mathrm{m}$ geometric diameter that corresponds approximately to the $1 \mu \mathrm{m}$ aerodynamic diameter used elsewhere in this paper and references made to submicrometer aerosol here will be based upon aerodynamic size.

[34] The number distributions (Figure 4b) from the C-130 and the normalized RB distributions (see corrections, Appendix B) are quite similar with integral numbers of $1215 \mathrm{~cm}^{-3}$ and $1125 \mathrm{~cm}^{-3}$ respectively and with a negligible contribution from particles larger than $0.7 \mu \mathrm{m}$. However, the RB tends to be somewhat broader with higher concentrations below $0.055 \mu \mathrm{m}$ and above $0.35 \mu \mathrm{m}$ but with lower values in between. When plotted as area distributions the indicated submicrometer integral values differ by less than $2 \%$ but the diameter of the peak in the C-130 is about $0.32 \mu \mathrm{m}$ and the RB data about $0.35 \mu \mathrm{m}$. However, the volume and surface area distributions also show larger differences above $1 \mu \mathrm{m}$. Even after corrections for CAI transmission efficiency (see Appendix A.1.3) the RB data shows 3 times more aerosol volume than the C-130. Some of this difference may reflect sampling losses in the sample line to the OPC or growth corrections for the APS. However, near surface coarse particle gradients and differences in sample times for the distributions may also contribute.

[35] The C-130 included NCAR wing probe measurements [Baumgardner et al., 1992] intended to supplement the onboard sizing data for the larger particles, expected to be poorly sampled with the Community Aerosol Inlet (CAI), and to characterize particles under ambient conditions. The wing probe data provided by NCAR from the FSSP and PCASP (ambient conditions) are also included in 

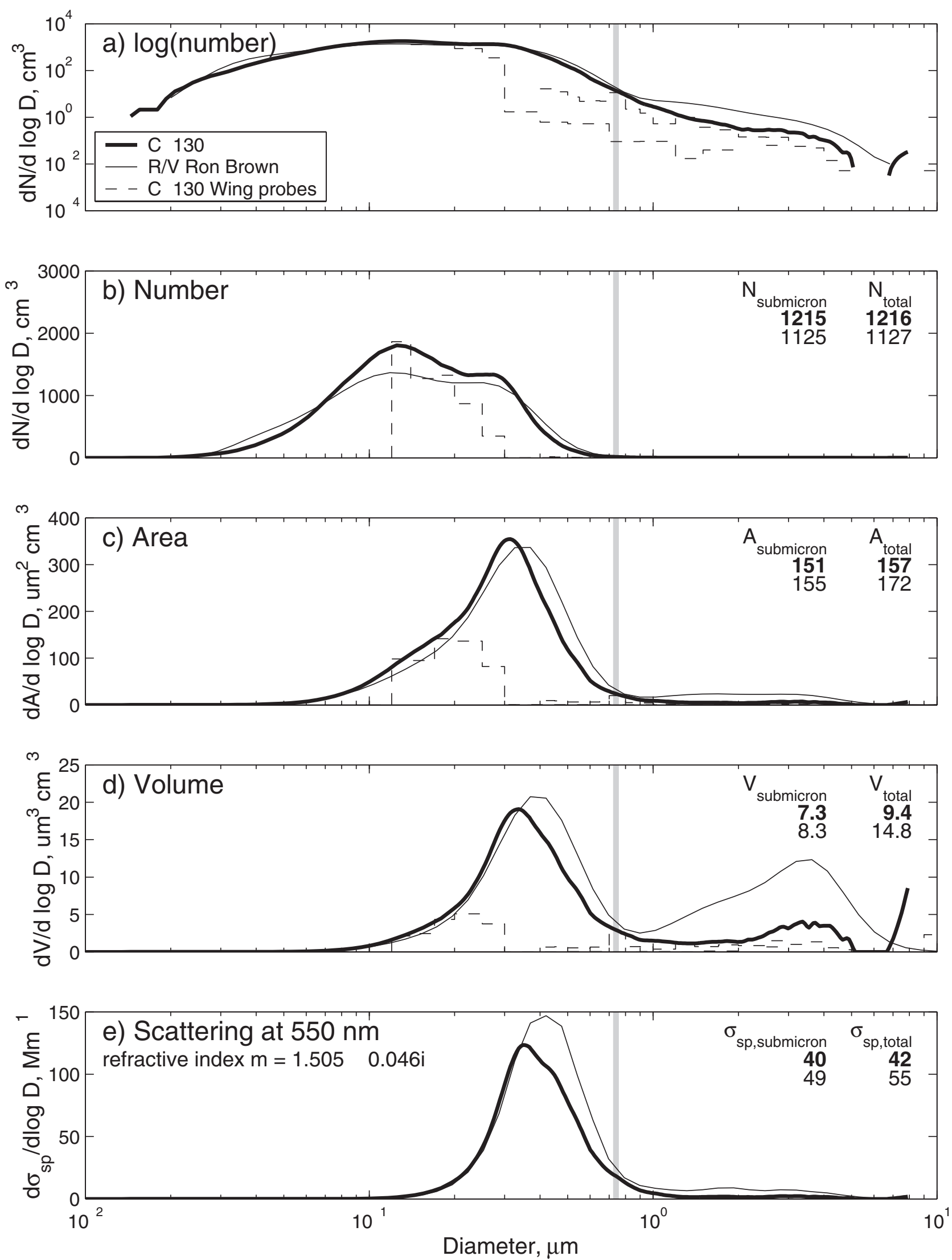

Figure 4. C-130 - RB Flyby Size Intercomparison for geometric diameters at 55\% RH. C-130 data as measured (about 6\% below C-130 CPC number) and RB data scaled (increased by 27\%) to match integral RB CPC number. Integral values for distributions of submicrometer and total aerosol shown on right of panels for $\mathrm{C}-130$ (bold) and RB (normal). 
Figures $4 a-4 d$. We note that these were based upon a refractive index of about 1.4 and have not been corrected to the lower RH of $55 \%$ used for the C-130 and RB data. These considerations should result in a reduction in size from that shown here and yield even larger differences between wing probe data and the other $\mathrm{C}-130$ and $\mathrm{RB}$ distributions than indicated here. PCASP data dominate the wing probe number distribution but the lower detection limit restricts it to above $0.12 \mu \mathrm{m}$ while severe undercounting is evident above about $0.23 \mu \mathrm{m}$. The area distribution also reveals that the FSSP data lie well below the C-130 and RB measurements for submicrometer sizes. Occasionally the wing probe data showed more consistent results than evident here but we were unable to identify conditions when this improved behavior could be predicted. Clearly, the wing probe data often provide unreliable characterization of the particle sizes between 0.2 and $1.0 \mu \mathrm{m}$ that dominate the scattering extinction distribution during INDOEX.

[36] The volume distributions for the indicated FSSP data for coarse particles are almost a factor of two below the C-130 OPC data and a factor of 6 or so below RB data. These low FSSP values could suggest that the much higher coarse particle concentrations evident in the $\mathrm{RB}$ data during the flyby may not be present at the C-130 altitude. However, this undercounting by the FSSP relative to the C-130 OPC was common for all cloud-free horizontal leg averages such that the quantitative interpretation of FSSP data under typical noncloudy conditions remains questionable. Hence, wing probe data cannot confirm whether the apparent coarse particle disparity between the C-130 and RB data during the flyby is real (e.g., a vertical or spatial gradient), instrumental or a sampling difference. Even so, the scattering distributions and their indicated integral values (Figure 4e) show that these larger particles contribute only $4.7 \%$ and $11 \%$ to the scattering extinction for the $\mathrm{C}-130$ and $\mathrm{RB}$ distributions respectively. This low relative contribution of coarse particles is also representative of most other surface data during INDOEX and C-130 data revealed this coarse component to generally decrease with altitude. Hence, sampling limitations of the CAI and possible limitations in C-130 coarse particle characterization compared to the RB should have little effect on the INDOEX optical characterization aboard the C-130.

\subsubsection{Sample Regime Differences}

[37] Size data have been grouped into L, M and $\mathrm{H}$ scattering regimes and different air mass regimes. However, the 23 low altitude legs on the C-130 often had only one or two legs that fell into one of these categories. Hence we will only compare C-130 and RB sizing for three of these groupings that included distributions from 3 or more 15 min C-130 flight legs with scattering values identified as AS-high, AS-medium and BoB high (Figures 5a and 5b). Because these measurements are not colocated the concentrations for the C-130 and RB platforms will vary significantly. Also, there are fewer samples from the C-130 due to its shorter time available for sampling opportunities under each condition. Even so, for each of these categories on the $\mathrm{C}-130$ and $\mathrm{RB}$, the size distributions and mean values is shown in Figures $5 \mathrm{a}$ and $5 \mathrm{~b}$ along with the mean values for both the number and volume distributions.

[38] In each of these three cases measured on both the C130 and $\mathrm{RB}$ there is frequent evidence of a bimodal accumulation mode number distribution that can be characterized with two lognormal distributions (not shown). The smaller and larger of these two components clearly fluctuate and may dominate the accumulation mode number. However, the submicrometer volume distribution is always determined by the larger of these modes and is described well by a single lognormal fit (see below).

[39] Although these RB and C-130 groupings are from different times and places certain features are common to both platforms. For the AS-medium case the number distributions show the greatest variability. The single unusually high number concentration in the smaller mode evident on the $\mathrm{C}-130$ was collected closest to India on our most northerly excursion. However, the corresponding mass volume distribution is close to the average indicating these enhancements in the smaller mode may influence the cloud condensation nuclei $(\mathrm{CCN})$ but contribute little aerosol mass or optical effect. All other C-130 distributions reflect the range of behavior also evident in the RB data. For the AS-high case (Figure 5) the distributions are similar to the AS-medium case but both the RB and C-130 data show greater dominance and fluctuations in the smaller of the two components of the bimodal accumulation mode number. A narrowing of the number distribution is also evident for both RB and C-130 AS-high data. The super-micrometer aerosol for the AS-high case is similar to the medium case for each platform, again reflecting the fact that the magnitude and variation in measured scattering is linked primarily to increases in accumulation mode volume. The BoB-high case in both $\mathrm{RB}$ and $\mathrm{C}$ 130 number distributions are more narrow and have relatively fewer coarse particles. The volume mean diameters on both platforms are also shifted to slightly larger sizes and suggesting a possible change in associated MSE values.

\subsection{Optical Properties}

[40] Comparisons of light scattering and light absorption were made for colocated measurements, e.g., including both UMiami and NOAA/CMDL had instruments at KCO and those periods when a mobile platform moved close to another mobile platform or to a stationary platform, e.g., C-130 flybys of KCO. The altitude of the flight segment was used to classify the segment as in or above the marine boundary layer (MBL) which was taken as $1 \mathrm{~km}$. Flight segments within the MBL were used in these comparisons. Additional interplatform comparisons were made for similar air mass regions and scattering ranges as described earlier.

[41] Table 5 summarizes the interplatform comparisons of intensive and extensive aerosol optical properties during times when the platforms were colocated. For colocated stationary platforms (e.g., KCO-UMiami and KCO-CMDL) measurements made over the same time period were compared. For mobile and stationary platforms (e.g., KCOCMDL and C-130) platform speed and wind speed were taken into consideration to ensure equivalent air masses were compared. A C-130 10 min flyby was typically compared to $90 \mathrm{~min}$ on either side of the flyby. During slow or near stagnant wind speed conditions the time for the air mass to pass KCO was longer than 3 hours but was reduced to $3 \mathrm{~h}$ to reduce problems associated with changes in wind direction and local contamination.

[42] Generally the measurements agree within 30\% but absorption measurements ( $\mathrm{Dp}<10 \mu \mathrm{m})$ made by NOAA- 

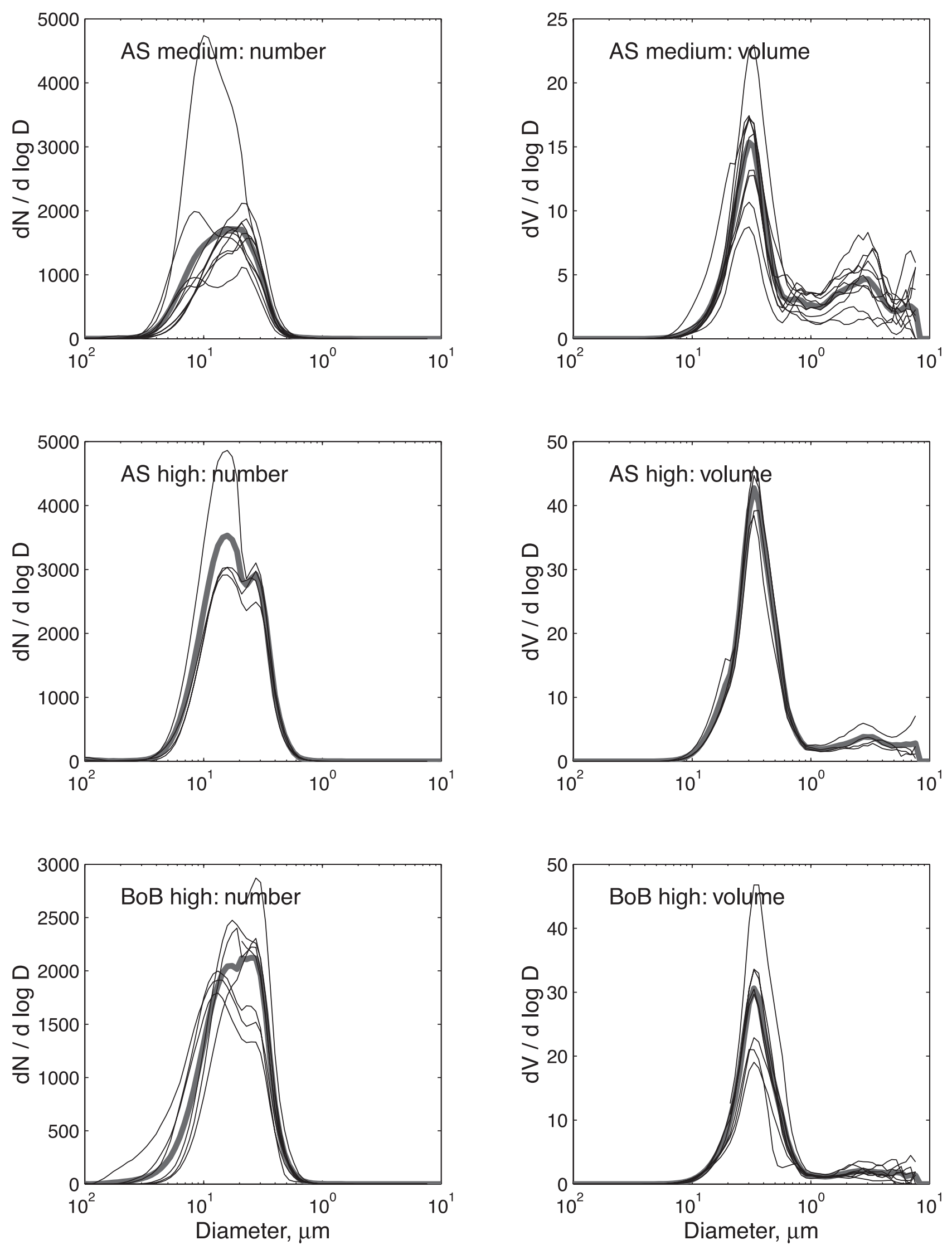

Figure 5a. Size distributions from C-130 for number and volume with geometric diameters at $55 \% \mathrm{RH}$. 

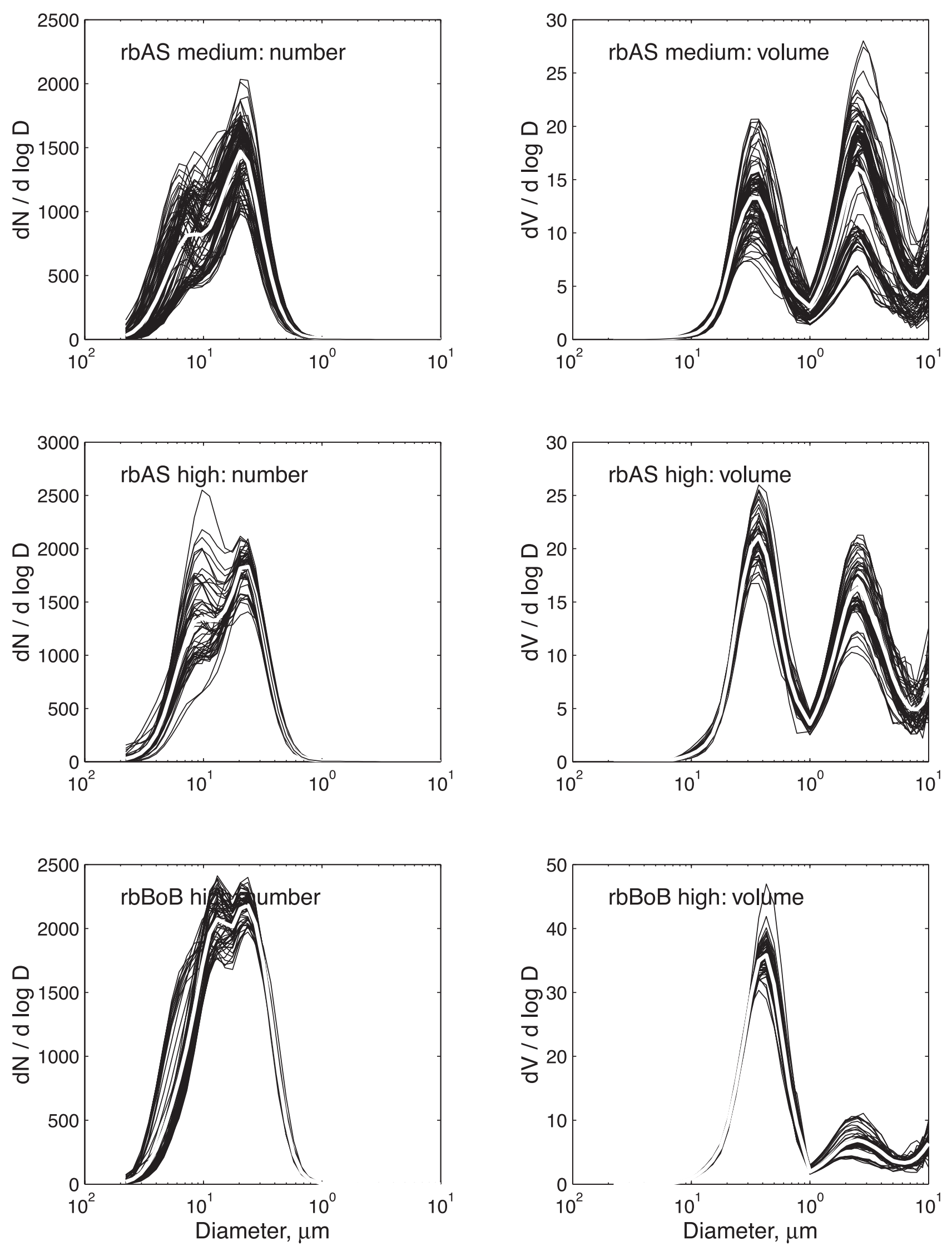

Figure 5b. Size distributions from RB for number and volume with geometric diameters at 55\% RH. 
Table 5. Ratio of $\mathrm{Dp}<1 \mu \mathrm{m}$ Aerosol Light Scattering Coefficients During Side-by-Side Comparisons ${ }^{\mathrm{a}}$

\begin{tabular}{lccll}
\hline & KCO-UMiami & KCO-CMDL & C-130 & RB \\
\hline & Light Scattering & Coefficient & & \\
KCO-UMiami & 1 & 1.20 & 1.40 & 1.64 \\
KCO-CMDL & & 1 & 1.17 & 1.48 \\
C-130 & & 1 & 0.94 \\
RB & & & & 1
\end{tabular}

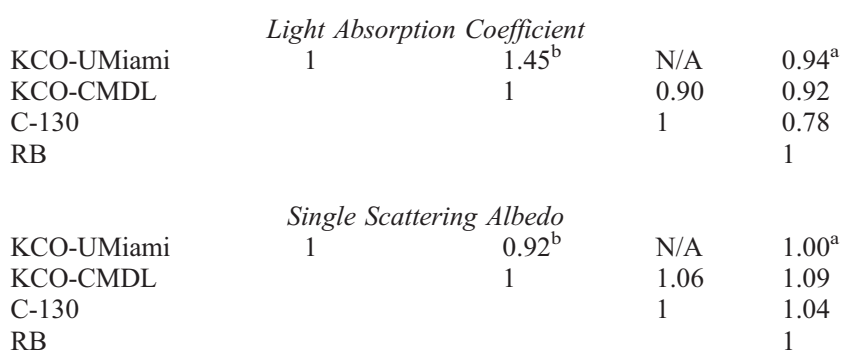

\begin{tabular}{|c|c|c|c|c|}
\hline \multicolumn{5}{|c|}{ Angström } \\
\hline KCO-UMiami & 1 & N/A & $\mathrm{N} / \mathrm{A}$ & N/A \\
\hline KCO-CMDL & & 1 & 1.10 & 1.08 \\
\hline $\mathrm{C}-130$ & & & 1 & 0.85 \\
\hline $\mathrm{RB}$ & & & & 1 \\
\hline
\end{tabular}

\begin{tabular}{|c|c|c|c|c|}
\hline \multicolumn{5}{|c|}{ Backscatter Fraction } \\
\hline KCO-UMiami & 1 & N/A & N/A & N/A \\
\hline KCO-CMDL & & 1 & 1.01 & 0.9 \\
\hline C-130 & & & 1 & 1.22 \\
\hline $\mathrm{RB}$ & & & & 1 \\
\hline
\end{tabular}

${ }^{a}$ Values from the platform given in the column headers are used in the numerator of all the ratios.

${ }^{\mathrm{b}}$ For Dp $<10 \mu \mathrm{m}$.

CMDL at KCO tend to be - higher (45\%) than the colocated measurements made by UMiami. It is unclear what this large difference can be attributed to - typically side-by-side comparisons of the PSAP instrument give results within $6 \%$ [Bond et al., 1999]. One known difference is the relative humidity (RH) at which the two instruments made their measurements. RH is known to affect the quality and noisiness of the PSAP measurements (P. Sheridan, personal communication, 2001). The humidity at the sample filters in the PSAP was likely to be higher than the reported system humidities (40\% KCO-CMDL, 55\% UMiami) because the metal filter holder was exposed to air-conditioned room air.

[43] Scatterplots (Figures 6a) of the UMiami and NOAA absorption, scattering and single scattering albedo for dp $<10 \mu \mathrm{m}$ (UMiami did not measure submicrometer absorption coefficients) show that there is good correlation between the two instruments, despite significant differences in the measurement values. A comparison of the single scattering albedo showed the two systems differed by about within $8 \%$, with the KCO-CMDL measurements showing lower albedos in keeping with the higher KCO-CMDL absorption coefficient and lower scattering measurement. Scattering coefficients measurements by the RB are significantly higher than either of the KCO-CMDL measurements, $48 \%$ higher than CMDL and 64\% higher than UMiami, although absorption measurements are within $10 \%$ and albedo comparisons are within $12 \%$ of the $\mathrm{KCO}$ values. The C-130 flybys of KCO yielded higher scattering values than either of the surface-based instruments at $\mathrm{KCO}$, but the absorption coefficient on the $\mathrm{C}-130$ was $10 \%$ lower than that measured by CMDL at KCO.

[44] Table 6 summarizes key intensive and extensive measurements in the Northern Hemisphere marine boundary layer for four platforms. Measurements made at the surface at KCO generally agree (within $15 \%$ ) with measurements made in the marine boundary layer by the $\mathrm{C}-130$. In contrast to the side-by side comparison of $\mathrm{KCO}$ measurements with the RB (Table 5), study-average extensive properties measured on the RB tend to be lower than both the C-130 measurements and KCO measurements. This may be because the RB transects sampled more air transported from cleaner regions (e.g., the northern Indian Ocean) than sampled by the C-130 in the MBL or KCO sampling stations.

[45] Table 7 summarizes measured submicrometer optical properties for the $\mathrm{L}, \mathrm{M}$ and $\mathrm{H}$ scattering regimes. Measurements of extensive properties by CMDL and UMiami at $\mathrm{KCO}$ had coefficients of variation (standard deviation/ mean) ranging from 0.15 to 0.32 , while $\varpi$ had coefficients of variation ranging from $0.025-0.05$, a factor of 4-6 smaller. While there are still large excursions in various parameters, including $\varpi_{0}$, it is unclear how much can be attributed to platforms sampling different air masses during the $\mathrm{L}, \mathrm{M}, \mathrm{H}$ regime comparisons as opposed to differences in sampling conditions (e.g., cut size, particle losses, etc.) The C-130 data show similar trends as the CMDL and UMiami measurements at KCO. Conversely, measurements aboard the RB suggest that as the scattering coefficient decreased the intensive properties of the aerosol also changed: the single scattering albedo increased consistent with a less absorbing (less polluted) aerosol, while the submicrometer Angström exponent decreased from values near 2.0 to about 1.4 and consistent with a larger (sea salt) aerosol [Delene and Ogren, 2001]. Aerosols with trajectories from the northern Indian Ocean (NIO) and southern Indian Ocean (SIO) fell into the "low" scattering category. Aerosols with trajectories originating over the Arabian Sea (AS) or Bay of Bengal (BoB) tended to have "medium" or "high" scattering characteristics, particularly if the air mass passed over India and/or Sri Lanka.

[46] Table 8 includes measured optical parameters for several platforms based on the starting point of air mass trajectories for the air sampled by the platforms. These comparisons suggest that air masses passing over the east side of India (i.e., over the Bay of Bengal) are more polluted (higher scattering and absorption coefficients, lower single scattering albedo) than air masses traveling on the west side of India (i.e., over the Arabian Sea). This result is consistent with the observed concentrations of nss sulfate and $\mathrm{BC}$ from the two regions.

[47] Table 9 groups aerosol optical properties as a function of latitude, where latitude is grouped into 3 categories: Northern Hemisphere, ITCZ and Southern Hemisphere. There is good agreement between the C-130 and the RB for the different regions, except for the absorption coefficients measured in the Northern Hemisphere. As would be expected, extensive properties decrease significantly along the north to south gradient and reflect the lower particulate pollution in the ITCZ and Southern Hemisphere. The single scattering albedo and backscatter fraction increase in the Southern Hemisphere, while the Angström exponent decreases, corresponding to a less absorbing, larger aerosol. 
A

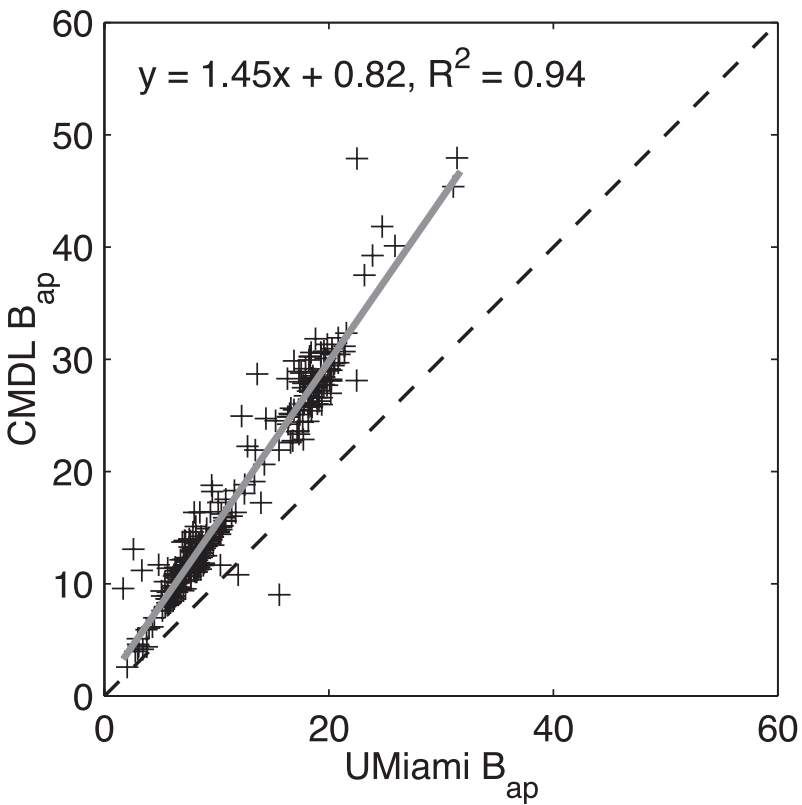

C

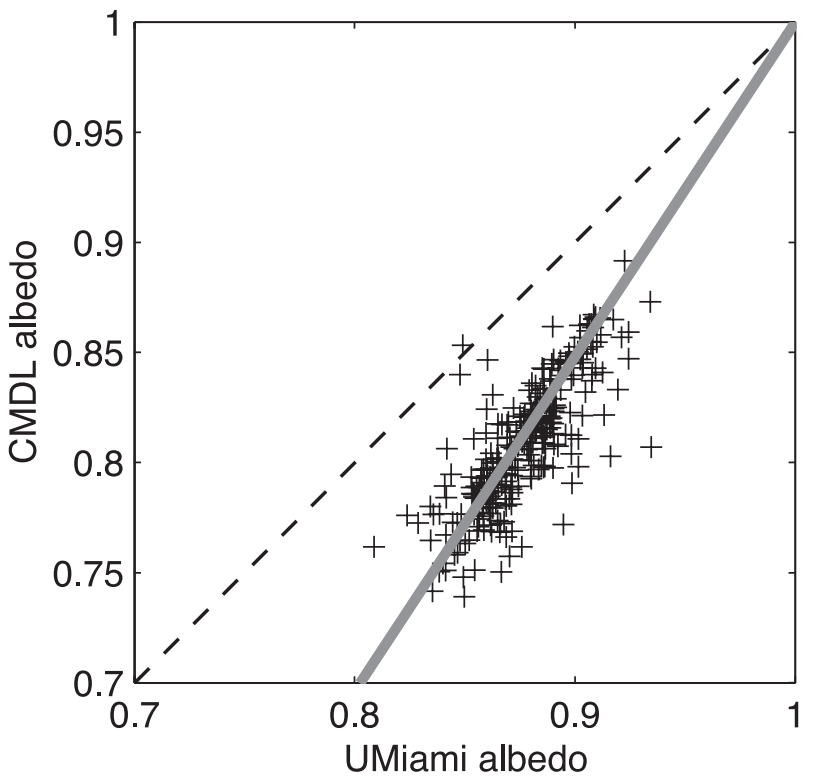

B

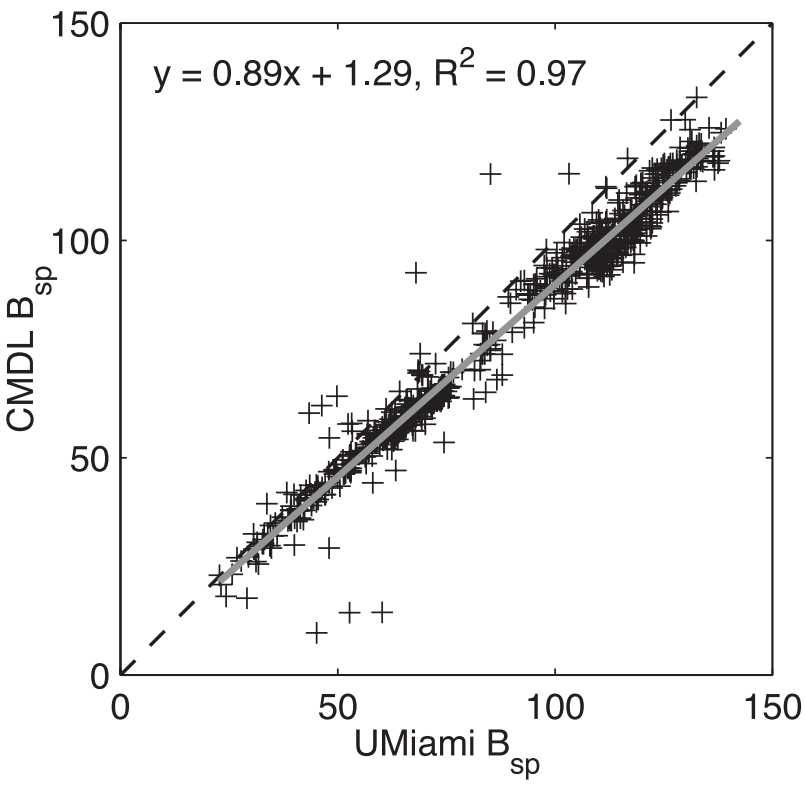

Figure 6. (a) Comparison of absorption coefficients $(\mathrm{Dp}<10 \mu \mathrm{m})$ measured at $\mathrm{KCO}$ by NOAA/CMDL and UMiami. (CMDL standard deviation $=8.8 \mathrm{Mm}^{-1}$ ). (b) Comparison of scattering coefficients (Dp $<10 \mu \mathrm{m})$ measured at KCO by NOAA/CMDL and UMiami. (CMDL standard deviation $=27.7 \mathrm{Mm}^{-1}$ ). (c) Comparison of albedo measured at KCO by NOAA/CMDL and UMiami (Dp $<10 \mu \mathrm{m})$. (CMDL standard deviation $=0.04$ ). Heavy grey line is relationship obtained for $\mathrm{CMDL}_{\text {albedo }}$ from slopes of regression lines for Figures $6 \mathrm{a}$ and $6 \mathrm{c}$ when forced through zero $\sim 1.5$ and 0.9 respectively). This yields expression $\mathrm{CMDL}_{\text {albedo }}=\left[1+(1.5 / 0.9)\left(\left(\mathrm{UM}_{\text {albedo }}\right)^{-1}-1\right)\right]^{-1}$.

The two platforms show the same trend for albedo (i.e., a $10 \%$ increase from the $\mathrm{NH}$ to the $\mathrm{SH}$ ). As mentioned below, the C-130 optical measurements were consistently higher than the RB for most regions compared. Additionally, the two platforms definition of the SH are very different - the RB spent a long time in the $\mathrm{SH}$ and was much further south than the $\mathrm{C}-130$. The $\mathrm{C}-130$ on the other hand spent much of its $\mathrm{SH}$ time in close proximity to the ITCZ and not much time in truly pristine SH air. This and the greater uncertainties associated with low values of scattering and absorption coefficients in the SH makes these intensive properties less robust.

\section{Discussion}

\subsection{Interplatform Differences}

\subsubsection{Chemical Properties}

[48] Variations in concentrations of all chemical species measured on the different platforms often exceeded 1 stand- 
Table 6. Ratios of $\mathrm{Dp}<1 \mu \mathrm{m}$ Extensive and Intensive Properties for Ground-Based and Aircraft Platforms in the Northern Hemisphere in the Marine Boundary Layer ${ }^{\mathrm{a}}$

\begin{tabular}{|c|c|c|c|c|c|}
\hline & $\begin{array}{c}\mathrm{C}-130 / \\
\text { KCO-CMDL }\end{array}$ & $\begin{array}{c}\text { C-130/ } \\
\text { KCO-UMiami }\end{array}$ & $\begin{array}{c}\mathrm{C}-130 / \\
\mathrm{RB}\end{array}$ & $\begin{array}{c}\mathrm{RB} / \\
\mathrm{KCO}-\mathrm{CMDL}\end{array}$ & $\begin{array}{c}\text { RB/ } \\
\text { KCO-UMiami }\end{array}$ \\
\hline$\sigma_{s p}$ & 1.01 & 1.24 & 1.51 & 0.41 & 0.51 \\
\hline$\sigma_{a p}$ & 0.86 & N/A & 2.12 & 0.67 & $0.82^{\mathrm{b}}$ \\
\hline$\omega_{0}$ & 1.05 & N/A & 0.93 & 1.12 & $1.03^{\mathrm{b}}$ \\
\hline$\AA(550 / 700)$ & 1.05 & $\mathrm{~N} / \mathrm{A}$ & 1.12 & 0.94 & N/A \\
\hline$B$ & 1.10 & N/A & 1.10 & 1.00 & N/A \\
\hline
\end{tabular}

ard deviation of the mean concentrations within scattering regimes, trajectory categories and geographical regions. In addition, interplatform comparisons often revealed considerable variability in the ratios of concentrations of chemical species. With the exception of $\mathrm{BC}$ to $\mathrm{TC}$ and $\mathrm{BC}$ to $\mathrm{OC}$, differences in mass ratios were often larger than 1 standard deviation of the means. This result is due in part to the variability in aerosol sources and transport pathways to the Indian Ocean coupled with airplane and ship tracks that did not allow for sampling of the same air mass. For example, BoB trajectories, which made up the majority of the high scattering regime, were sampled first by $\mathrm{KCO}$ and the $\mathrm{C}$ 130 (between 12 February and 3 March) and then by the RB (on 4, 5, and 29 March). Adding to the variability in chemical concentrations within the BoB trajectory category was geographical broadness. The category encompasses trajectories that followed a path from Calcutta over southern India or Sri Lanka to the Arabian Sea as well as trajectories that came from over central India to the Arabian Sea. Non-

Table 7. Values of $\mathrm{Dp}<1 \mu \mathrm{m}$ Extensive and Intensive Properties for Harmony Categories ${ }^{\mathrm{a}}$

\begin{tabular}{|c|c|c|c|c|}
\hline & KCO-CMDL & KCO-UMiami & $\mathrm{RB}$ & C-130 \\
\hline \multicolumn{5}{|l|}{$\sigma_{s p}$} \\
\hline High & $78.5 \pm 12.6$ & $61.4 \pm 15.2$ & $77.8 \pm 8.2$ & $85.4 \pm 22.97$ \\
\hline Medium & $42.8 \pm 9.0$ & $26.9 \pm 10.8$ & $40.3 \pm 14.0$ & $43.9 \pm 10.7$ \\
\hline Low (w/SIO) & $20.5 \pm 4.7$ & N/A & $12.0 \pm 5.6$ & $\begin{array}{l}19.7 \pm 6.7 \\
(13.3 \pm 10.2)\end{array}$ \\
\hline \multicolumn{5}{|l|}{$\sigma_{a p}$} \\
\hline High & $21.8 \pm 5.0$ & $17.8 \pm 4.9^{\mathrm{b}}$ & $13.0 \pm 1.6$ & $20.6 \pm 13.6$ \\
\hline Medium & $11.9 \pm 3.8$ & $7.7 \pm 3.1$ & $5.8 \pm 3.0$ & $9.52 \pm 9.9$ \\
\hline Low (w/SIO) & $6.3 \pm 1.9$ & N/A & $1.1 \pm 0.8$ & $\begin{array}{l}4.9 \pm 10.3 \\
(2.7 \pm 9.8)\end{array}$ \\
\hline \multicolumn{5}{|l|}{$\omega_{\mathrm{o}}$} \\
\hline $\begin{array}{l}\text { High } \\
\text { Medium }\end{array}$ & $0.78 \pm 0.02$ & $0.86 \pm 0.02^{\mathrm{b}}$ & $0.86 \pm 0.02$ & $\begin{array}{l}0.81 \pm 0.12 \\
0.86 \pm 0.36\end{array}$ \\
\hline Low (w/SIO) & $0.77 \pm 0.03$ & $\begin{array}{l}0.88 \\
\mathrm{~N} / \mathrm{A}\end{array}$ & $\begin{array}{l}0.88 \pm 0.04 \\
0.94 \pm 0.09\end{array}$ & $\begin{array}{l}0.80 \pm 0.36 \\
0.82 \pm 0.09\end{array}$ \\
\hline \multirow{2}{*}{\multicolumn{5}{|c|}{$\AA(550 / 700)$}} \\
\hline & & & & \\
\hline High & $1.92 \pm 0.08$ & N/A & $2.01 \pm 0.26$ & $2.08 \pm 0.29$ \\
\hline Medium & $1.94 \pm 0.08$ & & $1.83 \pm$ & $2.10 \pm 0.45$ \\
\hline Low (w/SIO) & $1.94 \pm 0.06$ & & $1.39 \pm 0.883$ & $\begin{array}{l}2.16 \pm 1.14 \\
(1.86 \pm 3.00)\end{array}$ \\
\hline \multicolumn{5}{|r|}{$(1000-200$} \\
\hline High & $0.10 \pm 0.003$ & N/A & $0.10 \pm 0.004$ & $0.10 \pm 0.02$ \\
\hline Medium & $0.11 \pm$ & & $0.10 \pm$ & $0.12 \pm 0.03$ \\
\hline Low (w/SIO) & $0.11 \pm 0.007$ & & $0.11 \pm 0.024$ & $\begin{array}{l}0.12 \pm 0.07 \\
(0.14 \pm 3.23)\end{array}$ \\
\hline
\end{tabular}

a"High" scattering $>55 \mathrm{Mm}^{-1} ; 25 \mathrm{Mm}^{-1} ;<$ "medium" scattering $<55$ $\mathrm{Mm}^{-1}$; and "low" scattering $<25 \mathrm{Mm}^{-1}$.

${ }^{\mathrm{b}}$ For $\mathrm{Dp}<10 \mu \mathrm{m}$. sea salt $\mathrm{SO}_{4}^{2-}$ concentrations measured on the $\mathrm{RB}$ were observed to vary by a factor of 3 within the BoB category depending on the exact path of the trajectory across the Indian subcontinent to the ship. This suggests that more specific tagging of aerosol properties in terms of potential source regions rather than general categories used here may be necessary to elucidate differences.

[49] For the carbonaceous aerosol components considered, $\mathrm{BC}$ and $\mathrm{OC}$, interplatform differences may also have resulted from nonstandardized sampling and analytical methods. Estimated concentrations of $\mathrm{BC}$ and $\mathrm{OC}$ depend on the measured concentration of total carbon and the method used to determine the $\mathrm{BC} / \mathrm{OC}$ split (see Appendix B). Although different methods result in comparable total carbon concentrations (within $\pm 20 \%$ ), estimated concentrations of BC and OC are more sensitive to the method used. In addition, estimated $\mathrm{OC}$ concentrations can be affected by positive (adsorption of gas phase organics) and negative (volatilization of semivolatile particulate organics) sampling artifacts [Turpin et al., 2000]. One approach for evaluating artifact-imposed differences in $\mathrm{BC}$ and $\mathrm{OC}$ concentrations involves an interplatform comparison of the $\mathrm{BC}$ to $\mathrm{TC}$ and light absorption to $\mathrm{BC}$ ratios. Consistency in these ratios across platforms suggests that artifact-imposed differences were not significant or were similar for all platforms.

Table 8. Values of $\mathrm{Dp}<1 \mu \mathrm{m}$ Extensive and Intensive Properties for Bay of Bengal and Arabian Sea Trajectories ${ }^{\mathrm{a}}$

\begin{tabular}{|c|c|c|c|c|}
\hline & KCO-CMDL & KCO-UMiami & $\mathrm{RB}$ & $\mathrm{C}-130$ \\
\hline \multicolumn{5}{|l|}{$\sigma_{s p}$} \\
\hline BoB & $64.3 \pm 25.3$ & $59.5 \pm 19.5$ & $87.3 \pm 5.0$ & $65.0 \pm 18.7$ \\
\hline AS & $59.1 \pm 21.5$ & $41.5 \pm 18.3$ & $38.5 \pm 4.5$ & $51.8 \pm 35.4$ \\
\hline \multicolumn{5}{|l|}{$\sigma_{a p}$} \\
\hline BoB & $18.2 \pm 7.6$ & $16.7 \pm 6.8^{\mathrm{b}}$ & $15.7 \pm 1.1$ & $17.4 \pm 11.5$ \\
\hline AS & $14.7 \pm 5.5$ & $12.1 \pm 5.7^{\mathrm{b}}$ & $5.4 \pm 0.9$ & $12.4 \pm 15.3$ \\
\hline \multicolumn{5}{|l|}{$\omega_{\mathrm{o}}$} \\
\hline $\mathrm{BoB}$ & $0.77 \pm 0.03$ & $0.86 \pm 0.02^{\mathrm{b}}$ & $0.85 \pm 0.02$ & $0.75 \pm 0.09$ \\
\hline AS & $0.80 \pm 0.02$ & $0.88 \pm 0.02^{b}$ & $0.89 \pm 0.01$ & $0.87 \pm 0.05$ \\
\hline \multicolumn{5}{|c|}{$\AA(550 / 700)$} \\
\hline BoB & $1.9 \pm 0.08$ & N/A & $2.09 \pm 0.25$ & $2.08 \pm 0.82$ \\
\hline AS & $2.01 \pm 0.03$ & & $1.75 \pm 0.13$ & $2.10 \pm 0.54$ \\
\hline \multicolumn{5}{|l|}{$B$} \\
\hline $\mathrm{BoB}$ & $0.10 \pm 0.006$ & N/A & $0.09 \pm 0.003$ & $0.11 \pm 0.05$ \\
\hline AS & $0.11 \pm 0.005$ & & $0.10 \pm 0.003$ & $0.11 \pm 0.04$ \\
\hline
\end{tabular}

${ }^{\mathrm{a}} \mathrm{BoB}$, Bay of Bengal; AS, Arabian Sea.

${ }^{\mathrm{b}}$ For $\mathrm{Dp}<10 \mu \mathrm{m}$. KCO-CMDL measurements do not extend beyond DOY 73.6 for $\mathrm{Dp}<1 \mu \mathrm{m}$; KCO-UMiami measurements do not extend beyond DOY 80 . 
Table 9. Values of Extensive and Intensive Properties for Submicrometer Aerosol in the Marine Boundary Layer as a Function of Latitude for the C-130 and RB

\begin{tabular}{lllllll}
\hline & \multicolumn{1}{c}{ RB NH } & \multicolumn{1}{c}{ C-130 NH } & RB ITCZ & C-130 ITCZ & RB SH & C-130 SH \\
\hline$\sigma_{s p}$ & $42.3 \pm 26.7$ & $63.27 \pm 28.4$ & $17.4 \pm 6.2$ & $20.7 \pm 19.0$ & $8.6 \pm 5.6$ & $6.41 \pm 6.07$ \\
$\sigma_{a p}$ & $6.8 \pm 5.3$ & $14.82 \pm 7.6$ & $5.15 \pm 4.2$ & $5.08 \pm 4.79$ & $0.7 \pm 0.4$ & $1.54 \pm 1.68$ \\
$\omega_{\mathrm{o}}$ & $0.88 \pm 0.04$ & $0.82 \pm 0.04$ & $0.90 \pm 0.05$ & $0.82 \pm 0.10$ & $0.98 \pm 0.03$ & $0.90 \pm 0.10$ \\
$\AA(550 / 700)$ & $1.80 \pm 0.40$ & $2.04 \pm 0.28$ & $1.17 \pm 0.34$ & $1.98 \pm 0.07$ & $1.11 \pm 0.64$ & $1.87 \pm 0.57$ \\
$B$ & $0.10 \pm 0.012$ & $0.11 \pm 0.01$ & $0.10 \pm 0.006$ & $0.11 \pm 0.02$ & $0.11 \pm 0.02$ & $0.14 \pm 0.04$ \\
\hline
\end{tabular}

[50] The uncertainty associated with collection and analysis of nss $\mathrm{SO}_{4}^{2-}$ typically is less than $\pm 10 \%$ [Quinn et al., 2000] so analytical effects were not expected to be a large contributor to interplatform differences in concentrations. In addition, differences in nss $\mathrm{SO}_{4}^{2-}$ concentrations between the $\mathrm{RB}$ and $\mathrm{C}-130$ are not a result of the correction required to derive nss $\mathrm{SO}_{4}^{2-}$ from total measured $\mathrm{SO}_{4}^{2-}$. The $\mathrm{Na}^{+}$ concentration used in the correction was similar for the two platforms and was about 2 orders of magnitude less than the nss $\mathrm{SO}_{4}^{2-}$ concentration. The difference also does not appear to be due to a difference in size cuts. Higher nss $\mathrm{SO}_{4}^{2-}$ concentrations would result if the RB sampler were collecting particles larger than the $\mathrm{C}-130$ sampler as more of the accumulation mode would be collected. In this case more $\mathrm{Na}^{+}$also would be collected by the RB sampler. But, for all scattering categories, $\mathrm{C}-130 \mathrm{Na}^{+}$concentrations were higher than those measured on the RB. NSS sulfate concentrations in the coarse mode were negligible (at least on the RB) compared to sea salt. Also, coarse mode $\mathrm{Na}$ concentrations were similar on the RB and C-130, not greater on the C-130.

[51] Different collection and analysis methods were used on each platform to determine the submicrometer aerosol mass concentration. In general, concentrations were highest for KCO-UMiami which employed an impactor with a broad size cut and KCO-Caltech whose sampler included a larger size fraction of the aerosol. Even though consistency in interplatform MSE ratios suggest analytical differences were not great, the observed variability in species mass concentrations and MSE were often greater than 1 standard deviation of the mean values. This result suggests that the aerosol measured by the different platforms had similar scattering per unit mass but different aerosol chemical composition. We hypothesize that the measured chemical composition differed primarily due to the many aerosol sources and transport pathways over the Indian Ocean, dissimilar platform sampling schedules, and the broadness of the categories the data was put into for comparison.

\subsubsection{Size Distributions}

[52] As mentioned earlier, the measured volume distributions encompass those sizes most effective optically in the INDOEX region and most of the optical effects are confined to the submicrometer accumulation mode. In Figure 7 the lognormal fits (dashed line) to the average volume distributions (solid line) for the C-130 and RB are compared for the AS-medium, AS-high, and BoB-high categories shown in Figure 5. The associated lognormal fit parameters to the volume distribution are summarized in Table 10. The calculated scattering distributions based upon the measured size data and the lognormal fits are similarly shown in the right hand panels and reveal that the fits produce essentially the same results as the measured distributions for the aerosol except for a small contribution by coarse aerosol that is also well described by a separate lognormal fit.

[53] The DMPS size distributions measured at KCO (W. Cantrell et al., Comparisons of calculated and measured CCN spectra at Kaashidoo Climate Observatory during INDOEX, submitted to Journal of Geophysical Research, 2000, hereinafter referred to as Cantrell et al., submitted manuscript, 2000) had mass mean diameters and spreads similar to both C-130 and RB distributions shown in Figure 5 but tended to vary more. The three C-130 flybys of KCO, where data were available to compare, all had volume mean diameters near $0.32 \mu \mathrm{m}$ on the $\mathrm{C}-130$ while $\mathrm{KCO}$ values varied from 0.32 to $0.35 \mu \mathrm{m}$ (not shown). Some of this difference is attributed to greater uncertainty in the KCO flow values and the operating $\mathrm{RH}$ (W. Cantrell, personal communication, 2000) but overall KCO sizes are consistent with the RB and C-130 data above.

[54] There is good agreement between the RB and C-130 size data and the main differences are a narrower distribution and fewer relative counts in the $0.35-0.8 \mu \mathrm{m}$ size range for the $\mathrm{C}-130$ data compared to the RB. This may be due to several reasons. First, the C-130 OPC is sensitive to socalled Mie-oscillations in the scattering properties of the aerosol in this size range [Garvey and Pinnick, 1983] that can lead to undercounting of the particles in this range and shifting them to other sizes. Also, there are differences in the approach used on the C-130 that employs assessment of measured aerosol growth (Figure 2) and chemically based refractive indices to adjust the measured "dry" size distributions while the RB employs a chemical model for growth. There are also uncertainties in RH values that come into play and the "rescaling" of the RB DMA data, as discussed in the appendix.

[55] In order to gauge the impact of uncertainties in key parameters that affect measurement of the size-distribution and related aerosol optical effects we have evaluated the effect on scattering extinction as we allowed these parameters to vary. The measured C-130 size-distribution from the $\mathrm{RB}$ flyby is taken as the reference distribution and random perturbations are made on sample flow rate, particle diameter, real and imaginary refractive index (as specified by RB measurements) and $\mathrm{RH}$ at the point of measurement. The variables, their variances, and the differential change in the resulting scattering are shown in Table 11. Scattering size distributions based on the combined OPC and DMA distributions were calculated 2000 times with input variables perturbed by normally distributed random amounts. The realization of these random perturbations are shown in Figure 8 along with the reference RB distribution (heavy 

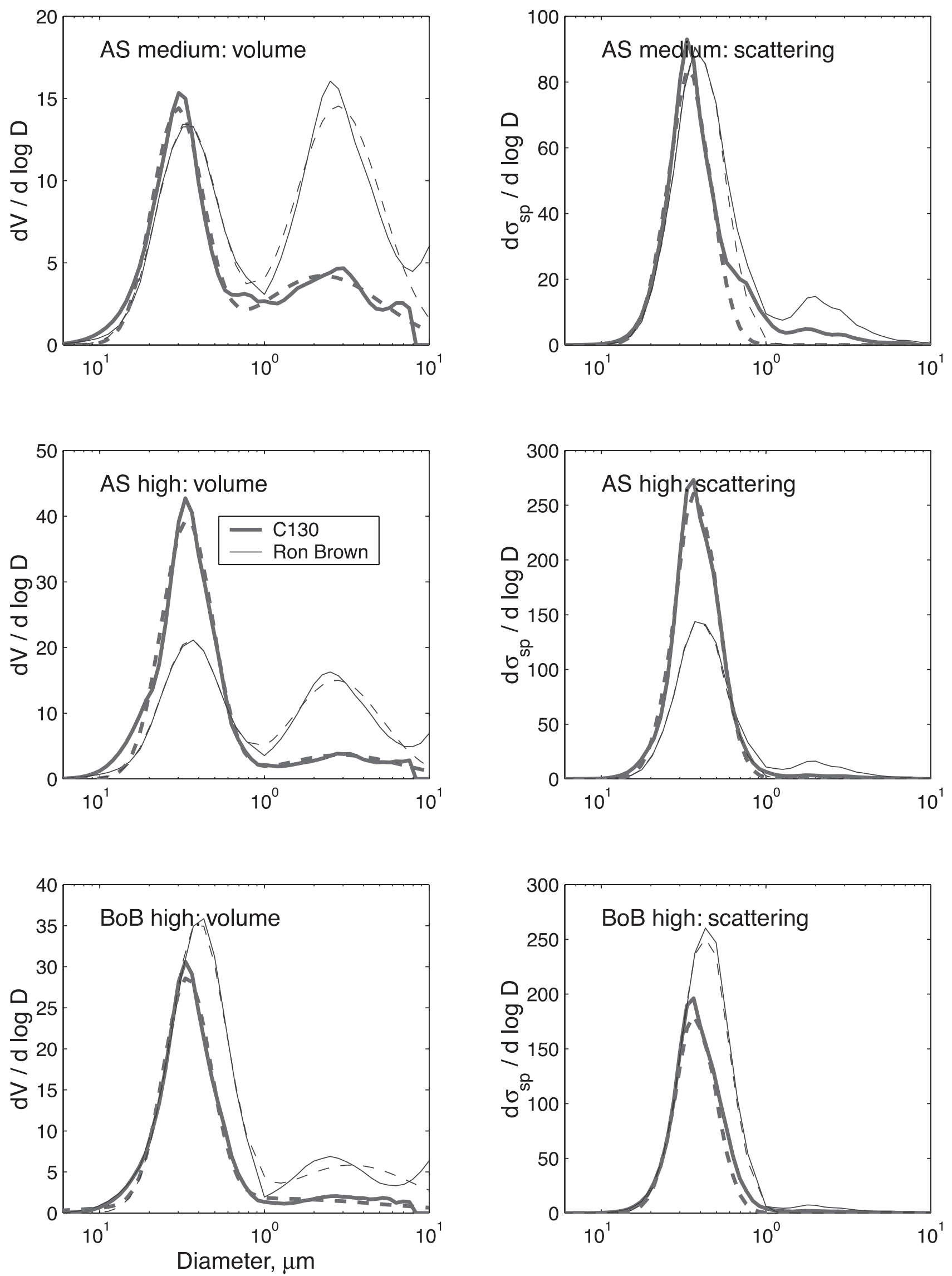

Figure 7. Measured and lognormal fit distributions for volume and scattering. Geometric diameter at $55 \%$ RH? 
Table 10. Lognormal Fits to Amplitude, Peak Diameter, and Standard Deviation for C-130 and RB Volume Distributions ${ }^{\text {a }}$

\begin{tabular}{llccccc}
\hline & Amp1 & Dp1 & Std D1 & Amp2 & Dp2 & Std D2 \\
\hline & \multicolumn{5}{c}{$C-130$} \\
AS medium & 5.5 & 0.30 & 1.42 & 3.8 & 2.3 & 2.28 \\
AS high & 15.2 & 0.34 & 1.43 & 3.3 & 2.9 & 2.28 \\
BoB high & 9.7 & 0.34 & 1.39 & 3.1 & 1.1 & 4.89 \\
& & & & & & \\
AS medium & 6.3 & 0.34 & 1.54 & 9.6 & 2.8 & 1.83 \\
AS high & 9.5 & 0.36 & 1.51 & 10.3 & 2.7 & 1.88 \\
BoB high & 15.1 & 0.40 & 1.48 & 5.7 & 3.4 & 2.44 \\
\hline
\end{tabular}

${ }^{\mathrm{a}}$ Diameter in $\mu \mathrm{m}$.

line). A range of one standard deviation for these perturbations is indicated by the dashed line, reflecting a $\pm 20 \%$ in integral scattering. The frequency histogram of integral scattering values is also included as an insert and suggests a tendency for more cases present with scattering above the most frequent value than below it. This is a consequence of the nonlinear dependency of scattering on particle size.

[56] As a test of "local" closure, the regression of measured and calculated light-scattering was done on both the RB and C-130 platforms using a Mie scattering code. On the RB, the size distributions and nephelometer were at the same humidity, so a size-dependent refractive index calculated from the aerosol composition during the RB flyby was used. A linear regression of 17 sampling periods yielded a slope of 1.07, an intercept of $1.37 \mathrm{Mm}^{-1}$, and a correlation coefficient of 0.98 . The lack of humidity control on the aircraft made the calculations somewhat more complicated. The $\mathrm{D} / \mathrm{D}_{\text {ref }}$ relationship from Figure 2 was used to adjust the OPC size distribution to the nephelometer humidity. The same size-dependent refractive index was used, but corrected for the difference in water mass between the nephelometer RH and the $55 \%$ of the RB sampler. Fortunately, these corrections were small. Scattering calculations for all 17 horizontal legs below $100 \mathrm{~m}$ where both the OPC and nephelometer sampled enough of the leg to get meaningful averages yielded a slope of 1.06, an intercept of $-2.18 \mathrm{Mm}^{-1}$, and a correlation coefficient of 0.98 . These can be compared to similar comparisons is extended to all INDOEX legs at all altitudes where we get correlation coefficients of 0.93 for submicrometer aerosol and 0.87 for total aerosol. Also, if we compare all calculated scatter from OPC sizes modeled at the same RH as the humidified nephelometer for all legs we get correlation coefficients of 0.84 for submicrometer aerosol and 0.81 for total aerosol.

Table 11. Effects of Size Distribution Error Modes on Calculated Scattering

\begin{tabular}{|c|c|c|c|}
\hline Variable & Variance & Scattering change & $\begin{array}{l}\text { Relative } \\
\text { Importance }\end{array}$ \\
\hline Diameter & $5 \%$ of diameter & $20 \%$ & 0.46 \\
\hline Flow rate & $3 \%$ of flow rate & $3 \%$ & 0.07 \\
\hline Real refr. index & $\begin{array}{l}10 \% \text { of diff. } \\
\text { from unity }\end{array}$ & $15 \%$ & 0.35 \\
\hline Imag. refr. index & $10 \%$ of value & $-0.4 \%$ & 0.01 \\
\hline $\mathrm{RH}$ at $\mathrm{OPC}$ & $3 \% \mathrm{RH}$ & $-5 \%$ & 0.12 \\
\hline
\end{tabular}

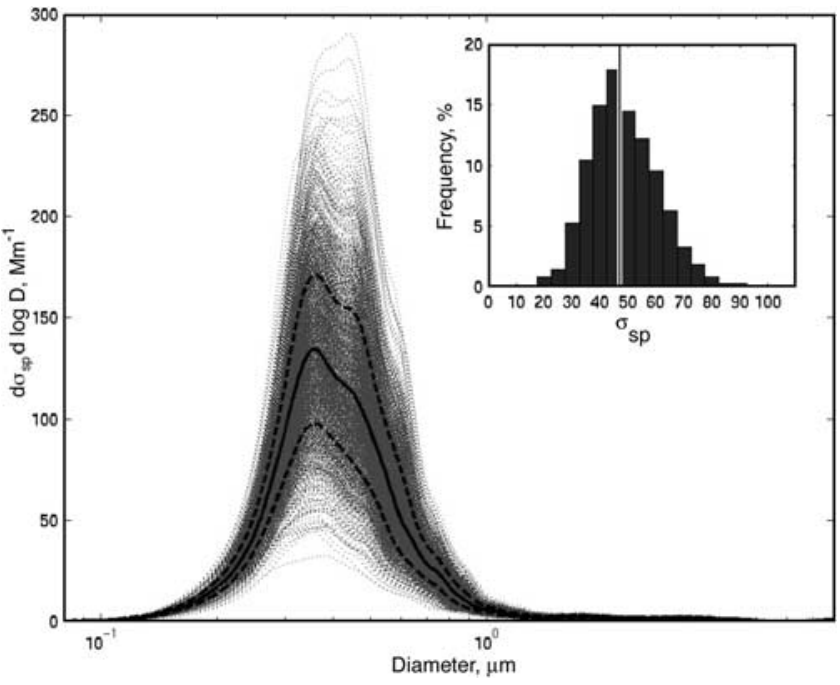

Figure 8. A Monte Carlo simulation of 2000 possible variations in the scattering distribution for the RB flyby for randomized uncertainties in various input parameters (see text). Dashed lines indicate one standard deviation about the reference distribution. Inset shows skewed frequency distribution of scattering around median value.

Hence, agreement between OPC calculated scattering and measured values are good for both wet and dry nephelometer measurements throughout the INDOEX campaign but are best for the low altitude data (with generally higher concentrations) compared here.

[57] Here we take size distributions from the C-130 and RB at the time of the RB flyby and used them to calculate scattering coefficients that can be compared to values measured in the C-130. Calculated and measured scattering for the three nephelometer wavelengths for periods when the nephelometer sampled both submicrometer $\left(\mathrm{D}_{\mathrm{a}}=1.0 \mu \mathrm{m}\right)$ and "total" aerosol $\left(D_{a}<10 \mu \mathrm{m}\right)$ are shown in Figure 9. For sample legs in the marine boundary layer, unexpected heater oscillations in the nephelometer sampling line periodically volatilized the incoming aerosol [Sheridan et al., 2002]. Hence, comparisons are only possible during the highlighted nephelometer measurement periods in Figure 9. Agreement is excellent for the C-130 calculated scattering for the submicrometer aerosol (ca. 0818 GMT) at all wavelengths while calculated values from RB size distributions are about $10 \%$ higher. We believe this indicates that submicrometer aerosol on the C-130 are effectively sampled and measured both by the nephelometer and by the aerosol sizing instrumentation such that local "closure" between measured and calculated values is achieved. The somewhat higher calculated RB scattering values are well within the range of uncertainty (ca. 10\%) in either the measurement or even the possible natural aerosol variability in measurement region.

[58] When sampling "total" aerosol (ca. 0828 GMT) the C-130 calculated scattering values are about 10\% lower than measured while values calculated from RB sizes are somewhat higher than measured C-130 values (Figure 9). We expect that this is may be a result of three possibilities. The first is coarse particle sample line losses between the CAI inlet that are greater for the $\mathrm{C}-130 \mathrm{OPC}$ measurement than for the nephelometer resulting in poorer estimates of scatter for 


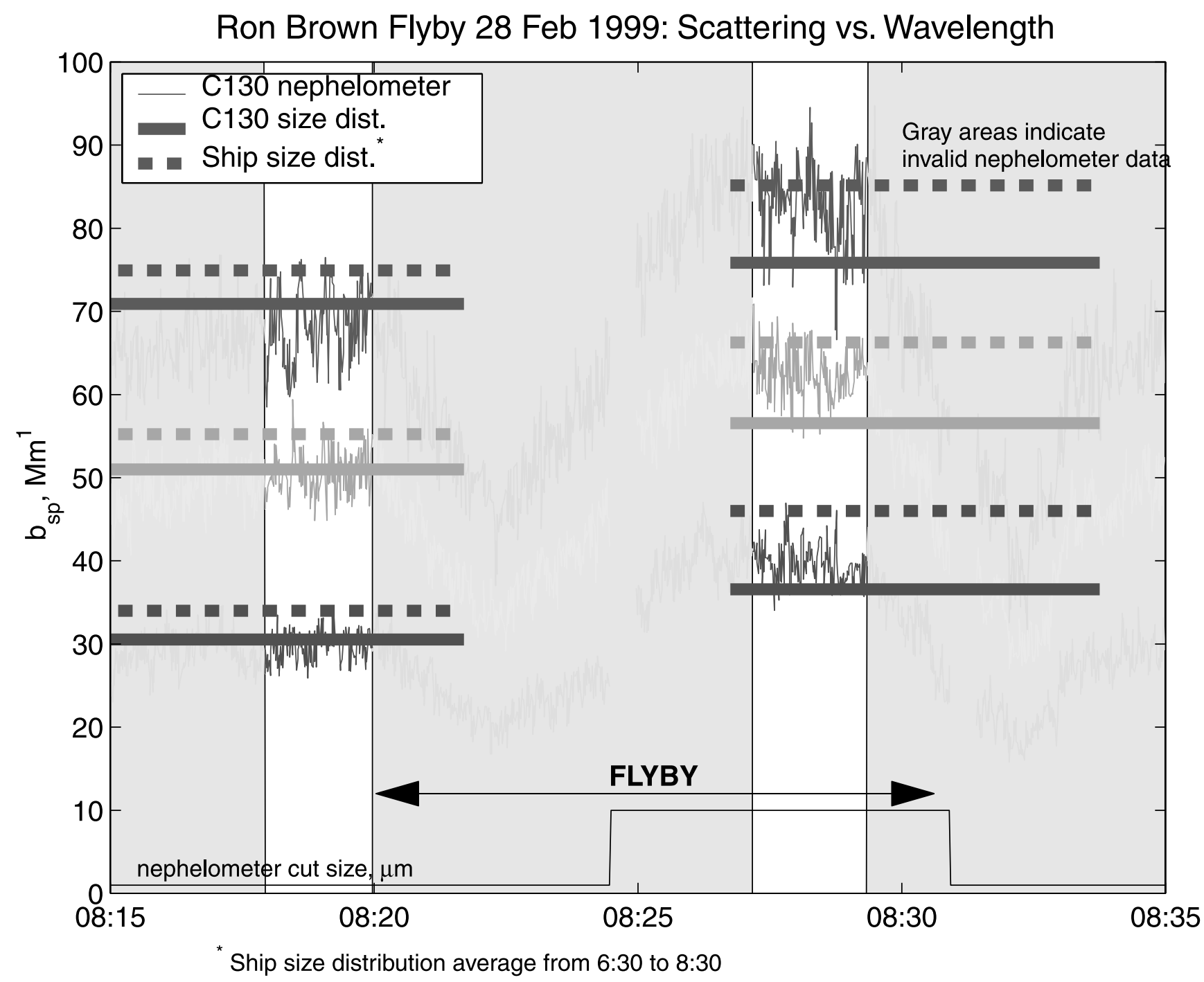

Figure 9. Light scattering values measured (C-130) and calculated from size distributions during RB flyby. The first time period is for submicrometer aerosol and the second is for total aerosol. See color version of this figure at back of this issue.

the larger OPC sizes. The flow path to the OPC is more complex due to the thermal analysis system and the inlet to the OPC is not designed to transmit particle larger than $7 \mu \mathrm{m}$ effectively while the nephelometer has large bore and shorter tubing with little bends. The second is better sampling of coarse aerosol by the RB than by the $\mathrm{C}-130$ as suggested by coarse size differences in Figures $5 \mathrm{a}$ and $5 \mathrm{~b}$. A third could be overestimates of growth corrections for the RB APS. Even so, the differences in size distributions and associated optical properties for both RB and C-130 measurements during the flyby are small and within predicted uncertainties.

\subsubsection{Optical Properties}

[59] All platforms during INDOEX measured considerable variability in aerosol light scattering and absorption. Some of this variability may be attributed to meteorological influences (e.g., rain) but the greatest changes were associated with changes in the characteristics of the source region of the aerosol. Even within the polluted Northern Hemisphere, there were times when light scattering fell into the Low scattering category. Even so, aerosol intensive properties were much less variable with greatest variations evident along the north-south gradient. Moving from Northern Hemisphere to Southern Hemisphere there was a factor of 5 to 10 decrease in absorption and scattering that corresponded with a $10 \%$ increase in albedo. For comparison, the shift in trajectory regime from $\mathrm{BoB}$ to $\mathrm{AS}$ in the Northern Hemisphere resulted in less than 5\% increase in albedo, while absorption and scattering decreased by less than a factor of 3 . The Northern Hemisphere $\mathrm{H}$ and $\mathrm{L}$ scattering regimes, which showed shifts in extensive properties ranging from factors of 4 to 6 , showed small differences in albedo $(\sim 1 \%)$ except for the RB measurements. These suggested a $9 \%$ differences in albedo for the RB's more extensive measurements in the $\mathrm{L}$ scattering regime.

\subsubsection{Consistency of Measurements}

[60] In spite of specific cases of good "local" closure mentioned above, general comparisons among the C-130, KCO-UMiami and KCO-CMDL showed that the light scattering and absorption coefficients measured by these platforms would differ by $30 \%$. Extensive and intensive 
aerosol optical properties measured by the $\mathrm{C}-130$ and $\mathrm{KCO}$ CMDL were in better agreement, typically within $15 \%$, for most comparisons but larger than expected based upon established instrument performance.

[61] KCO-UMiami measured lower scattering (20\%) and absorption $(45 \%)$ values than the CMDL package at $\mathrm{KCO}$, while scattering measured on the RB tended to be higher than either of the KCO platforms for side-by-side comparisons. This could be due to in part to a $20 \%$ difference in the amount of submicrometer scattering aerosol (calculated from $\sigma_{s p}(\mathrm{Dp}<1 \mu \mathrm{m}) / \sigma_{s p}(\mathrm{Dp}<10 \mu \mathrm{m})$ for these platforms: KCO-CMDL had a study average submicrometer fraction of 0.66 while KCO-UMiami had a submicrometer fraction of 0.51 . The RB had a study average submicrometer fraction of 0.75 which could also explain the higher RB scattering values. (Note: The submicrometer absorbing fraction of the aerosol measured by KCO-CMDL was 0.84 .) Uncertainty in size cut may contribute to this discrepancy. However, using the Caltech data for density as a function of $\mathrm{RH}$ expected geometric cut-size diameters were determined to be $0.72 \mu \mathrm{m}$ at $\mathrm{RH}=40 \%$ and $0.75 \mu \mathrm{m}$ at $\mathrm{RH}=55 \%$ for the $\mathrm{KCO}, \mathrm{RB}$ and C-130 impactors. Comparisons with possible scattering distributions in Figure 7 would suggest that this small difference is unlikely to be enough to explain the higher RB measurements unless the cut occurred at smaller sizes and/or was broader than expected. For example, to explain the $20 \%$ difference in submicrometer scattering, we assumed the KCO-CMDL impactor operated as designed (size cut $=0.72 \mu \mathrm{m}$ and $\mathrm{RH}=40 \%$ ) and used Figures 2 and 7 to estimate operating conditions for the UMiami impactor. The UMiami impactor size cut would have to be $0.59 \mu \mathrm{m}$ and the RH resulting in that size cut $\sim 70 \%$ in order to explain this submicrometer scattering difference. These values are far from the designed system parameters that, while they do not eliminate RH-influenced size cut as a factor, they do suggest other factors contribute to the observed differences.

\subsubsection{Consistency of Intensive Parameters}

[62] Intensive parameters $\left(\varpi_{0}, b\right.$, å) were typically within $20 \%$ of each other in spite of the much larger differences for extensive parameters. This difference between the variability of intensive and extensive properties may be expected for situations where mixing of clean and polluted air is the source of the variability. For example if two different air masses (clean air: $\sigma_{s p}=5 \mathrm{Mm}^{-1}, \varpi_{\mathrm{o}}=0.95$; polluted air: $\sigma_{s p}=100$ $\mathrm{Mm}^{-1}, \varpi_{\mathrm{o}}=0.80$ ) were mixed the scattering coefficient could vary by a factor of 20 as the mix varied from clean to polluted but the maximum variation in albedo would be less than $20 \%$. Other processes that affect extensive properties such as precipitation scavenging or particle losses in inlets, can change intensive properties only if they discriminate against particles with different size or composition.

[63] We can hypothesize three possible explanations for differences in measurements: (1) differences in the ambient aerosol; (2) differences in the sampled aerosol; (3) differences in how the instruments work. Instrument uncertainties can only explain some of the differences in the side-by-side comparisons. Previous side-by-side comparisons of 2 nephelometers have shown that when sampling the same air they agree within 1\% for submicrometer particles [Anderson and Ogren, 1998]. Comparisons of 3 PSAPs in parallel have shown that these instruments can agree within $4 \%$ when measuring the same aerosol [Bond et al., 1999], although Anderson et al. [1999] noted that instrument noise in the PSAP can be significant and should be determined for each instrument individually. The analysis described in Table 4, shows that the uncertainty in light scattering measurements for a TSI nephelometer is $5.2 \mathrm{Mm}^{-1}$ for submicrometer scattering measurements in the $50 \mathrm{Mm}^{-1}$ range, equivalent to $10 \%$ uncertainty. This is not enough to explain why submicrometer scattering measured by CMDL at KCO was $20 \%$ greater than the scattering measured by UMiami at $\mathrm{KCO}$, or why the scattering measured on the RB was $48 \%$ greater than the scattering measured by CMDL at KCO. We do not have a definitive explanation for the differences, although it seems unlikely that the third hypothesis (differences in how the instruments work) is entirely responsible. However, the other two hypotheses remain unresolved: (1) that the aerosols were actually different, due to spatial or temporal variations; or (2) that sampling inlets and/or impactors had different size-dependent sampling efficiencies. The first cannot account for the CMDL/UMIAMI differences at $\mathrm{KCO}$ while the second might play a role if size classification is less certain than expected. Additionally, these intercomparisons suggest that stratifications based on $\mathrm{L}, \mathrm{M}$, and $\mathrm{H}$ scattering categories may obscure variability due to sources or aerosol character.

\section{Summary of Indoex Aerosol Properties}

\subsection{Chemical Properties}

[64] Although concentrations of chemical species varied between platforms, for the individual platforms all concentrations of $\mathrm{BC}, \mathrm{OC}, \mathrm{TC}, \mathrm{nss} \mathrm{SO}_{4}^{2-}$, and submicrometer aerosol mass increased with a shift from the low to medium to high scattering regime. For example, at $\mathrm{KCO}$, on the $\mathrm{C}$ 130 , and on the $\mathrm{RB}$, nss $\mathrm{SO}_{4}^{2-}$ mean concentrations in the high scattering regime were a factor of $3.2,12$, and 5.2 higher, respectively, than mean concentrations in the low scattering regime. In addition, for the two mobile platforms, mean concentrations of nss $\mathrm{SO}_{4}^{2-}$ were a factor of $8(\mathrm{RB})$ to 25 (C-130) higher for the Northern Hemisphere (latitudes north of $1^{\circ} \mathrm{S}$ ) than for the Southern Hemisphere (latitudes south of $5^{\circ} \mathrm{S}$ ). Measurements of $\mathrm{BC}, \mathrm{OC}, \mathrm{TC}$, and aerosol mass on the $\mathrm{RB}$ followed the same trend with decreasing concentrations as the ship moved from the Northern Hemisphere, to the ITCZ (latitudes between $1^{\circ} \mathrm{S}$ and $5^{\circ} \mathrm{S}$ ), and to the Southern Hemisphere.

[65] When the Northern Hemisphere air masses are separated into trajectories coming from the $\mathrm{AS}$ and those coming from the BoB, trends observed by the C-130 and $\mathrm{RB}$ are quite different, however. The C-130 mean concentrations of nss $\mathrm{SO}_{4}^{2-}$ were higher in the $\mathrm{AS}$ than the $\mathrm{BoB}$ air masses while $\mathrm{BC}, \mathrm{OC}$, and $\mathrm{TC}$ mean concentrations were similar. $\mathrm{RB}$ mean concentrations of $\mathrm{nss}^{-2-}, \mathrm{BC}, \mathrm{OC}$, and $\mathrm{TC}$ were all higher in the BoB than the AS air masses. These differences reflect the variability in transport pathways and resulting aerosol concentrations within the AS and $\mathrm{BoB}$ trajectory classifications and the different sampling schedules of the two platforms.

[66] Unlike the absolute mass concentrations, within each platform submicrometer mass ratios of the chemical species (BC to TC, $\mathrm{OC}$ to nss $\mathrm{SO}_{4}^{2-}$, and nss $\mathrm{SO}_{4}^{2-}$ to aerosol mass) were relatively consistent across scattering regimes. In 
addition, $\mathrm{BC}$ to $\mathrm{TC}$ ratios were similar for all platforms

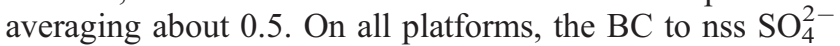
mass ratio increased with a shift from the low to medium to high scattering regime indicating more $\mathrm{BC}$ relative to sulfate in the more polluted air masses. Mean ratios of nss $\mathrm{K}^{+}$to $\mathrm{BC}$ were higher on the $\mathrm{RB}$ than on the $\mathrm{C}-130$ for the medium and high scattering regimes. Based on these observed ratios, it appears that the $\mathrm{RB}$ detected a greater or more frequent influence of biomass burning emissions on the aerosol chemical composition than the C-130. Mean scattering to mass ratios (at $33 \% \mathrm{RH}$ ) measured at $\mathrm{KCO}$ and on the RB were consistent between platforms and scattering regimes ranging from 3.4 to $4.4 \mathrm{~m}^{2} \mathrm{~g}^{-1}$. Ratios based upon IC and carbon analysis from the C-130 were about $40 \%$ higher due largely to the use of the sum of chemically analyzed mass instead of gravimetrically determined mass for the total mass concentration. Absorption to $\mathrm{BC}$ ratios are more variable between platforms but still within 1 standard deviation of the mean values. Mean ratios showed no trend with scattering regime and ranged from 9.2 to $20 \mathrm{~m}^{2} \mathrm{~g} \mathrm{C}^{-1}$ across all platforms and scattering regimes.

\subsection{Microphysical Properties}

[67] The size distribution of the INDOEX aerosol that dominated mass and optical properties was submicrometer accumulation mode aerosol. This was predominantly an internal mixture [Ramanathan et al., 2001] of soot-like aerosol mixed with the chemical species discussed above. The measured number distributions on the RB and C-130 were quite variable with frequently a smaller and larger number mode contributing to the accumulation mode (ca. $50 \mathrm{~nm}$ to $700 \mathrm{~nm}$ ). The volume distribution and optical properties were dominated by the larger and less variable components (ca. $150 \mathrm{~nm}$ to $700 \mathrm{~nm}$ ). Integrated size distributions on the $\mathrm{C}-130$ agreed with total CPC measured number and revealed negligible concentrations below CPC lower limit size detection. This supported the scaling of number distributions on the RB to CPC total number, as found necessary due to flow fluctuations in RB sizing instruments. When the data from both platforms were stratified into regimes, aerosol submicrometer number distributions exhibited similar variations in each classification, suggesting that the measurements and variations seen on both platforms were representative. Though more limited and more variable, DMA size distributions also measured at KCO near the time of C-130 flybys varied within the fluctuations shown here for the RB and C-130 distributions.

[68] At a typical measurement $\mathrm{RH}$ of $55 \%$ the accumulation mode volume mean diameter was generally near 0.34 $\mu \mathrm{m}$ and with a spread (sigma) of about 1.4. A separate coarse mode component with a volume mean diameter of about $2.7 \mu \mathrm{m}$ generally included sea-salt and at times dust or fly ash but usually had a small effect on light scattering. Even after correction for super-micrometer particle transmission efficiencies in the C-130 CAI inlet the coarse particle concentrations appeared to be lower by a factor of 3 or so compared to the RB. Although near surface gradients in species such as sea-salt could not be ruled out, it is probable that coarse particle transmission losses between the CAI and the $\mathrm{C}-130$ sizing instruments are responsible for much of this difference.
[69] Wing probe size distributions aboard the C-130 in the noncloud environment often trended in number with onboard size distributions but usually failed to capture the peak in the accumulation mode distributions and tended to undercount the internal probes for all size ranges. Coupled with often erratic and unpredictable variability these wing probe data were generally not suitable for determination of aerosol optical properties.

[70] A Monte-Carlo simulation of perturbations to the calculated scattering caused by uncertainties in the size distribution at 55\% RH was shown to have greatest sensitivity to characterization of diameter $(45 \%)$ and the real refractive index $(35 \%)$. At this $\mathrm{RH}$, sensitivity in calculated scattering due to possible uncertainty in measured RH was only about $12 \%$ but this can be expected to become much greater at higher RH typical of boundary layer ambient conditions. Even so, the combined random errors possibly affecting the size distribution measurements showed that $66 \%$ of the 2000 realizations for calculated scattering distributions fell within $\pm 25 \%$ of the reference scattering value. However, due to the nonlinear dependency of light scattering on size the random fluctuations about the mean characteristics tend to result in more frequent higher scattering values than lower scattering values. This suggests that random errors in measurements affecting size could tend to slightly overestimate derived scattering values in the mean.

[71] Light scattering properties were very well described by lognormal fits to the volume size distribution on both the C-130 and RB. In the high scattering events in the Arabian Sea the lognormal fit parameters at $55 \% \mathrm{RH}$ for volume accumulation mode diameter were $0.34 \mu \mathrm{m}$ and $0.36 \mu \mathrm{m}$ with spreads (standard deviation) of 1.43 and 1.51 for C130 and $\mathrm{RB}$ distributions respectively. This reflects the tendency to slightly smaller diameters and narrower peaks in the C-130 data. Both platforms suggest a slight increase in aerosol diameter with concentration.

\subsection{Optics}

[72] Considerable variability in all optical properties was seen across the different comparisons. Surprisingly, the side-by-side comparisons were not significantly better than the scattering regime and air mass comparisons, despite the added benefit of the platforms being colocated. For example, the RB measured scattering 48 and 64\% higher than KCO-CMDL and KCO-UMiami during side-by-side measurements, while scattering regime and trajectory comparisons of scattering were within 50\%. Differences in inlet and sampling conditions (e.g., RH, Dp) may have contributed to observed differences in the side-by-side comparisons, while additional variability was introduced by the sampling of different air masses for scattering regime and trajectory comparisons. Consistent with the chemistry measurements, there was less variability among intensive properties than extensive properties.

[73] Northern Hemisphere extensive properties during polluted conditions (e.g., $\mathrm{H}$ scattering regime and BoB trajectory) ranged from $60-85 \mathrm{Mm}^{-1}$ for scattering and between 15-22 $\mathrm{Mm}^{-1}$ for absorption. Single scattering albedo values were between 0.77 and 0.86 , while backscatter fraction and Ångström exponent were fairly invariant at 0.10 and 2.0 respectively. Southern Hemisphere values are available for the $\mathrm{RB}$ and $\mathrm{C}-130$, although the statistics 


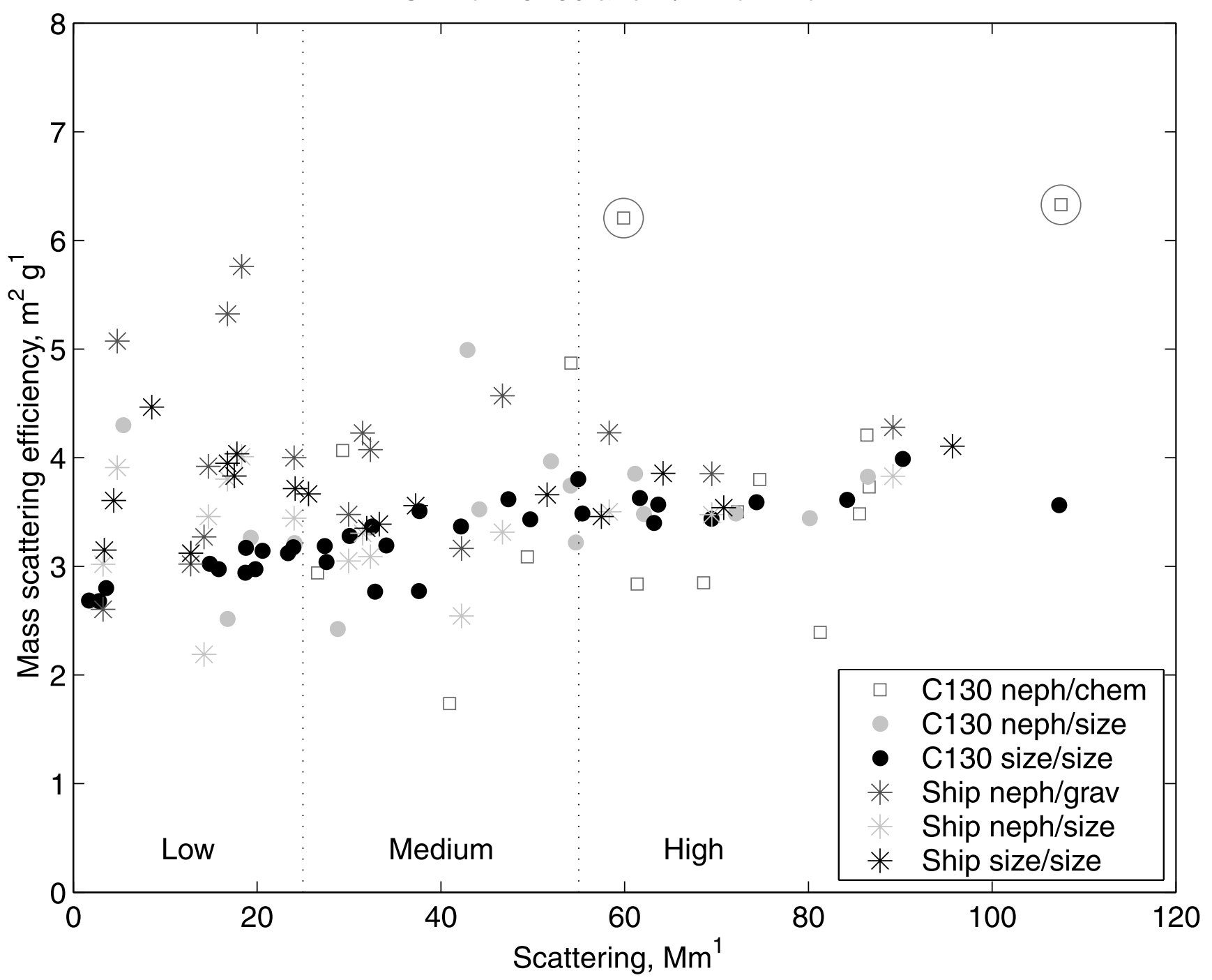

Figure 10. MSE values as a function of scattering values for various measurements (see text). See color version of this figure at back of this issue.

for the $\mathrm{C}-130$ are limited because it was not in the SH for long. Further hampering the comparison is the consistent difference mentioned above between the RB and C-130 even for the more robust Northern Hemisphere measurements. Nonetheless, Southern Hemisphere extensive properties tended to be 5 to 10 times less than values measured in the $\mathrm{NH}$, while single scattering albedo increased by about $10 \%$ over values measured in the $\mathrm{NH}$.

\subsection{Comparison of Mass Scattering Coefficients}

[74] The various measurements discussed previously provide a general understanding of the characteristics and uncertainty present in an experiment of this kind. Certain values, particularly intensive properties, are a valuable tool for modelers and others to employ. The mass scattering extinction ratio (MSE or specific scattering extinction) is important because it provides a link between mass and optical properties. However, as shown here, both mass and optical properties can be derived from a variety of measurements. The extent to which values are seen to agree provides an indication that "closure" is being achieved and that confidence can be placed in the values so derived.

[75] In Figure 10 we have compared six approaches for obtaining submicrometer MSE at 33\% RH from the C-130 and $\mathrm{RB}$ as a function of the measured scattering with the low, medium and high ranges indicated. These include various definitions of MSE based upon mass and scattering values corrected to $33 \% \mathrm{RH}$. The six definitions are: measured scattering to gravimetrically measured mass (RB), measured scattering to mass estimated from size distributions ( $\mathrm{RB}$ and $\mathrm{C}-130$ ), scattering to mass ratios determined solely from size distributions (RB and C-130) and measured scattering to filter extracted chemically determined mass (C-130) after correction for associated water at $33 \% \mathrm{RH}$. Note that circled $\mathrm{C}-130$ values are identified as outliers in mass measurements [Mayol-Bracero et al., 2002]. In order to have one consistent definition of MSE in Figure 10, it was necessary to add an estimate of the water mass expected at $33 \% \mathrm{RH}$ to the mass determined by chemical analysis aboard the $\mathrm{C}-130$. This was done as out- 
lined in the discussion of Figure 2 above by assuming that dry density was $2.2 \mathrm{~g} / \mathrm{ml}$ and that all additional volume as the measured particles grow $14 \%$ in diameter is due to water.

[76] Several features of the MSE values in this plot are summarized here for the indicated L, M and H categories:

[77] HIGH scattering category representative of the most concentrated INDOEX haze plumes: (1) About $80 \%$ of all values fall between about 3.4 and $4.0 \mathrm{~m}^{2} \mathrm{~g}^{-1}$. (2) Values based upon RB gravimetric mass are about $15 \%$ higher than corresponding values based upon RB size distributions. (3) Values based upon measured scattering and size derived mass are indistinguishable for both RB and C-130 and not significantly different from MSE values estimated from size alone. This reveals the good agreement between measured size and scattering found independently on both platforms.

[78] MEDIUM scattering category representative of moderate INDOEX pollution events: (1) Values based on gravimetric mass tend to have increased variability and remain more elevated compared to other MSE measurements. (2) Values based upon measured scattering and size derived mass remain similar but with increased variability (both RB and C130) and not very different from values based exclusively on size. There is also a tendency for all but gravimetric values to decrease as scattering values decrease.

[79] LOW scattering category representative of diluted continental and/or "clean" MBL air: (1) A general increase in variability in MSE for all measurements, as might be expected for ratios of measurements at low concentrations where signal to noise is greater. (2) The tendency for several gravimetric based MSE values on the RB to significantly exceed both MSE values based upon size suggests the greater uncertainty likely for gravimetric data obtained at lower concentrations. Variability is least for MSE values based exclusively on size since numerator and denominator are obtained from the same instrument measurement. (3) Tendency for MSE data to be greater on RB than C-130. [Note: MSE values for C-130 collected for altitudes above $30 \mathrm{~m}$ (not shown) were consistently higher than the values shown here and similar to average values for RB data in this category.]

[80] The tendency for values from all approaches to converge under the high scattering regime where signal to noise issues are less of an influence provides confidence that the MSE for the INDOEX haze is well constrained. This suggests MSE values from each platform is representative of the haze. The gradual decrease in MSE values ongoing from high toward low scattering conditions evident in most measurements is probably real since uncertainties in single particle sizing instruments on both the RB and C-130 are insensitive to concentration changes compared to gravimetric or even nephelometer measurements. The greater variability of MSE in Figure 10 as determined from chemical composition is unsurprising, as the mass is the sum of several individual measurements, some of which are inevitably close to or below detection limits given the short sampling times possible on the aircraft. However, some variability is expected due to changes in aerosol properties and air mass types.

\section{Conclusions}

[81] Concentrations of chemical species measured on the different platforms were highly variable within scattering regimes, trajectory categories, and geographical regions. These interplatform differences often were greater than 1 standard deviation of the mean concentrations for a particular category. In addition, comparisons between platforms revealed large variability in the mass ratios of chemical species. With the exception of $\mathrm{BC}$ to $\mathrm{TC}$ and $\mathrm{BC}$ to $\mathrm{OC}$ ratios, differences in mass ratios were larger than 1 standard deviation of the mean values. Although analytical differences may contribute to the observed variability, consistent interplatform scattering to mass ratios and absorption to mass ratios imply that analytical differences were not great. We hypothesize, therefore, that these differences also reflect the variability in aerosol sources and transport pathways to the Indian Ocean coupled with airplane and ship tracks that did not allow for sampling of the same air masses. Making "round robin" chemical intercomparisons of different analysis techniques for the same sample and appropriate sideby-side platform comparisons should be a high priority in future multiplatform experiments and would help to differentiate analytical variance from natural variance.

[82] Although variations in size distributions were marked on each platform and for each category discussed here the "shape" of the distributions in terms of mean lognormal diameters and widths were found to be quite consistent for all platforms. The RB accumulation mode also tended to indicate a slightly larger volume mean diameter than measured on the $\mathrm{C}-130$ that is probably a result of Mie scattering effects in the $\mathrm{C}-130$ OPC near 0.6 $\mu \mathrm{m}$. Even so, lognormal fits to the measured volume mode on both platforms provided excellent representation of associated light scattering and supports using this approach to modeling optical effects in terms of size and composition. The RB flyby was the only case of "local closure" for measured scattering and modeled values between the RB and C-130 platforms (based upon size and measured chemistry) but showed very good agreement in derived scattering extinction with differences less than $20 \%$. However, Monte Carlo simulations (2000 realizations) were carried out for realistic random variations in scattering distributions derived from variations in sizing measurement parameters for this "flyby" distribution. These realizations (Figure 8) resulted in variability of one standard deviation representing a $\pm 25 \%$ variation in derived extinction. They also revealed that the frequency of distribution about the reference scattering value was skewed from a normal distribution. The most frequently occurring simulated value was about 3\% less than the reference value. Also, the fall off in frequency of occurrence for larger scattering values was more gradual than for smaller scattering values (i.e., unusually large realizations were more evident than unusually small realizations).

[83] Coarse particle volume measured with the APS was generally higher on the RB than both OPC and wing probe data on the $\mathrm{C}-130$ by a factor of 2 or so, even after correcting OPC data for CAI sampling efficiency. This may be due to large particle losses in the longer and more convoluted sample lines to the OPC inside the aircraft and/ or possible uncertainties in APS coarse particle behavior (see Appendix B). In view of the dominance of accumulation mode aerosol for INDOEX aerosol optical properties, these differences for $\mathrm{RB}$ and $\mathrm{C}-130$ coarse particle contributions to extinction in were shown to have less than $8 \%$ 
influence on near surface optical properties (see Figure 4) and even less for column average values due to generally more rapid fall off in coarse particle aerosol with height. Wing probe data was found to be generally unreliable for determining accumulation mode optical characteristics but was more consistent at larger sizes where, as discussed, contributions to overall optical properties were small.

[84] Intensive and extensive aerosol optical properties observed during INDOEX demonstrated several trends across platforms: (1) aerosol properties were representative of clean air in the Southern Hemisphere and more polluted air in the north hemisphere, while the ITCZ aerosol had properties between the two; (2) air mass trajectories from the Bay of Bengal tended to be more polluted than air from the Arabian Sea (3) categories of high, medium and low scattering were also periods of high, medium and low absorption, although intensive properties across platforms did not show a pattern consistent with differing contributions of pollution aerosol for each category. Additionally, all platforms measured absorption coefficients in the Northern Hemisphere higher than typically reported at US rural continental sites, resulting in low albedo observations (range 0.78 to 0.94 ) for the INDOEX study region. Despite consistent trends among platforms, quantitative comparisons of extensive properties showed significant differences (up to $60 \%$ ) even for colocated instruments (e.g., KCO-UMiami and RB) and up to a factor of two for identical instrument packages (e.g., KCO-CMDL, C-130, $\mathrm{RB}$ ) within $\mathrm{L}, \mathrm{M}$ and $\mathrm{H}$ scattering regimes. Intensive properties generally agreed within $20 \%$ for all scenarios studied.

[85] We note that the best overall agreement was found between the C-130 and KCO-CMDL measurements (with similar plumbing and RH conditioning) which typically agreed within $15 \%$ for both extensive and intensive properties. Differences between identical instruments in slightly different packages (e.g., RB and KCO-CMDL) during sideby-side comparisons are particularly disturbing. Known measurement uncertainties cannot explain the observed differences in intensive and extensive properties. Hence, it seems likely that instruments were sampling different aerosols due to temporal or spatial variations or that individual sampling variations resulted in different subsets of the atmospheric aerosol being measured. Also, stratification based solely on ranges of scattering coefficient (e.g., the L, M, H categories) exhibits variability in optical properties in each range that suggests differences in regional and aerosol characteristics.

[86] Although the high correlations found between measured and modeled scattering on the RB and C-130 are good examples of "local closure" some of the differences just discussed in the ratios of chemical species and in optical properties evident between platforms are greater than one would expect. In spite of this, the various determinations of MSE ratios presented for the RB and C-130 under the higher haze conditions (where measurements are most robust) and at $33 \% \mathrm{RH}$ were shown to be about $3.8 \pm 0.3$ $\mathrm{m}^{2} \mathrm{~g}^{-1}$. However, at lower concentrations MSE values are more varied. This probably reflects a combination of the greater measurement uncertainties at low concentrations and real variations in aerosol properties. This low variability in MSE values both between techniques and in various loca- tions is well within the range expected for possible $10 \%$ uncertainties in measured scattering coefficients and 20\% possible uncertainties in modeled size distribution properties discussed in the text. Also, since several of the uncertainties assumed (e.g., uncertainty in an RH sensor or measurement of particle diameter by a particular instrument) are often be systematic and not random this $20 \%$ uncertainty may be an upper limit. However, we believe that without great effort to ensure sensor and measurement performance on all platforms during experiments like INDOEX it will be difficult to claim "closure" with an uncertainty much less than this. Even so, the data discussed here provides our best understanding of the chemical, microphysical and optical properties of aerosol in the INDOEX region and provides significant constraints upon the interpretation and modeling of aerosol properties for the region. At the same time, the variability in the average aerosol intensive properties evident on each platform and within the various categories discussed here points out the difficulty of describing "typical" conditions for even a single intensive sampling campaign. This underscores the long-term need to link and extend such assessments to satellite observations that can provide measurements over the spatial and temporal scales needed to reliably interpret regional and global impacts.

\section{Appendix A. \\ A.1. Platforms}

[87] The intensive phase of INDOEX involved near surface measurements from five platforms that were usually active in different regions at different times, often employed different measurement approaches, different analytical techniques, different sample inlet configurations and different sampling conditions. A brief description of sampling systems on the various platforms provides a starting point for assessing potential differences that might impact a consistent description of the INDOEX aerosol. Since effective comparison of similar measurements on the Sagar Kanya [Jayaraman et al., 2001] was not carried out we will not discuss that data here.

\section{A.1.1. RB Sampling Approach}

[88] RB sample air was drawn through a $6 \mathrm{~m}$ sample mast with an entrance $18 \mathrm{~m}$ above sea level and forward of the ship's stack. To maintain nominally isokinetic flow and minimize the loss of super-micrometer particles, the inlet was manually rotated into the relative wind. Air entered the inlet through a $5 \mathrm{~cm}$ diameter hole, passed through an expansion cone, and then into the $20 \mathrm{~cm}$ diameter sampling mast with a flow of $1 \mathrm{~m}^{3} \mathrm{~min}^{-1}$. The last $1.5 \mathrm{~m}$ of the mast were heated to establish a reference $\mathrm{RH}$ for the sample air of $55 \pm 5 \%$. This allows for constant size segregation and comparable measurements in spite of variations in ambient RH. Individual $1.9 \mathrm{~cm}$ diameter stainless steel tubes extended into the heated portion of the mast and were connected to the aerosol instrumentation and impactors with graphite-polyethylene conductive tubing or stainless steel (impactors) to prevent the electrostatic loss of particles. Air was sampled only when the concentration of particles greater than $15 \mathrm{~nm}$ in diameter indicated the sample air was free of local contamination (i.e., there were no rapid increases in particle concentration), the relative wind speed 
was greater than $3 \mathrm{~m} \mathrm{~s}^{-1}$, and the relative wind was forward of the beam.

\section{A.1.2. KCO Sampling Approach}

[89] Chemical, physical, and optical measurements were made at the Kaashidhoo Climate Observatory (KCO) on Kaashidhoo Island $\left(4.96^{\circ} \mathrm{N}, 73.47^{\circ} \mathrm{E}\right)$ in the Republic of Maldives. During the early field campaign (11 February to 26 February 1999) aerosol samples were collected over consecutive 48 -hour periods by the California Institute of Technology (Caltech). Two filter samplers and four microorifice uniform deposit impactors (MOUDI, MSP Corp., Model 100) were operated simultaneously on the roof of the observatory's laboratory building at an elevation of $3 \mathrm{~m}$ above ground level. Samples were collected at ambient temperature (typically $27-29^{\circ} \mathrm{C}$ ) and RH (typically $80-$ $87 \%$ ) for 46 hours out of every 48 hours, starting at $6 \mathrm{pm}$ local time every second day. The University of Miami (UMiami) collected daily samples for aerosol chemical composition using a Sierra impactor operating at ambient $\mathrm{RH}$ located at the top of the tower on the roof of the laboratory building over the entire field campaign.

[90] Instruments located inside $\mathrm{KCO}$ employed the UMiami designed stack and inlet [approx. $13 \mathrm{~m}$ asl] system shared with several other investigators, including NOAA/ CMDL. An omnidirectional inlet [Liu et al., 1983] was mounted atop a $10 \mathrm{~m}$ tall, $13 \mathrm{~cm}$ ID vertical PVC pipe with a $2001 \mathrm{~min}^{-1}$ maximum laminar flow rate (tube Reynolds number $<2000$ ). The intake pipe passed through the roof and connected to a $13 \mathrm{~cm} \times 13 \mathrm{~cm} \times 5 \mathrm{~cm}$ sidearm PVC pipe tee with a central $5 \mathrm{~cm}$ OD thin wall aluminum heater tube in order to keep the sample air warm and below about $60 \% \mathrm{RH}$. This restricted the $\mathrm{RH}$ range to where humidityinduced particle growth was relatively small and also helped to avoid water condensing inside the instrument systems due to the lower temperature in the air-conditioned KCO. At the bottom of the heater the flow split for sampling by the different instruments and excess air maintained the 2001 $\min ^{-1}$ flow through the intake system. A flow of $21 \mathrm{~min}^{-1}$ was taken out horizontally through a $\sim 1 \mathrm{~cm}$ stainless steel tube to the UMiami Particle/Soot Absorption Photometer (PSAP) and $101 \mathrm{~min}^{-1}$ was drawn straight down into the UMiami impactors and nephelometer system. The UMiami nephelometer was also heated to maintain the humidity close to $55 \%$, although their PSAP was not heated. Particle sizing included a DMA system (Cantrell et al., submitted manuscript, 2000) employing the same UMiami inlet system and a dry sheath flow but continuous RH measurements were not recorded. Losses by turbulence, diffusion and inertia were calculated to be less than $5 \%$ for this inlet system. Potential static charge losses were not calculated for the large diameter PVC pipe but are expected to be small for sizes influencing optical properties.

[91] The NOAA/CMDL aerosol system extracted 301 min $^{-1}$ from the aerosol sampling stack through a $1.9 \mathrm{~cm}$ OD stainless steel sample tube inserted just down stream of the stack's inlet heater. To reduce RH to $\sim 40 \%$, CMDL further heated the air stream prior to sampling it in their instruments. Prior to entering the CMDL nephelometers and PSAP the air was directed through either a 1 or $10 \mu \mathrm{m}$ impactor to provide information about aerosol size. Flow was switched between impactors every $6 \mathrm{~min}$. For both the UMiami and KCO-CMDL systems, the measured nephel- ometer scattering was adjusted to $55 \%$ based on CMDL's $\mathrm{f}(\mathrm{RH})$ measurements if $\mathrm{RH}$ measured at the nephelometer differed from $55 \%$.

\section{A.1.3. C-130 Sampling Approach}

[92] The near surface C-130 measurements were typically 10 to $20 \mathrm{~min}$ flight legs flown at about $35 \mathrm{~m}$ or higher altitude, consistently higher than the other platforms. Typical airspeed of $120 \mathrm{~m} \mathrm{~s}^{-1}$ resulted in between 70 and 140 $\mathrm{km}$ of air being sampled on a 10-20 min leg. Most instruments sampled through the so-called community aerosol inlet (CAI) built by NCAR [Blomquist et al., 2001]. This 8 $\mathrm{m}$ isokinetic probe extended beyond the nose of the C-130 and was designed to gradually slow flow while shedding sample tube boundary layer air in order to suppress turbulence and particle losses. The CAI was designed to provide a single inlet source to multiple investigators but an evaluation of the CAI efficiency in the Community Aerosol Inlet Evaluation Experiment (CAINE-2) showed that the CAI worked well for submicrometer particles, but its efficiency dropped rapidly above that, with a $50 \%$ cut size in the $2-3$ $\mu \mathrm{m}$ range [Blomquist et al., 2001]. More recent and detailed inlet tests carried out as part of the PELTI inlet evaluation experiment (July 2000) also revealed that the CAI had the greatest particle losses of the 3 inlets tested and confirmed the need for large corrections for particle sizes above $1 \mu \mathrm{m}$ [Huebert et al., 2000]. Fortunately, on almost all legs flown on the C-130 during INDOEX the aerosol number, surface area, mass and associated light-scattering/absorption was dominated by the submicrometer accumulation mode aerosol. Exceptions were in clean regions dominated by sea salt and occasional cases with an elevated dust component.

[93] Various inlet tubes left the CAI to instrument racks near the front of the aircraft with sample line lengths that varied from about $1 \mathrm{~m}$ to $4 \mathrm{~m}$. Low flow instruments employed bypass flows to minimize particle sedimentation. Due to the rapid changes in altitude and both outside and cabin temperatures, $\mathrm{RH}$ was less readily controlled on the $\mathrm{C}-130$ than on other platforms and most investigators simply monitored RH at key locations so corrections to target humidities could be made later.

\section{Appendix B.}

\section{B.1. Chemical Characteristics}

\section{B.1.1. Species Considered}

\section{B.1.1.1. Sample Collection}

[94] The methods of sample collection for determining aerosol chemical composition are listed in Table A1 for each platform. A Berner-type multijet cascade impactor was used for sample collection on the RB and operated at $55 \pm$ $5 \%$ RH. For the collection of samples for ion and gravimetric analysis, a jet plate with a $50 \%$ aerodynamic cut-off diameter, $D_{50 \text {,aero }}$ of $1.1 \mu \mathrm{m} \pm 0.11$ was used. Sampling times ranged from 4 to $6 \mathrm{~h}$ for ions and $12 \mathrm{~h}$ for mass. Samples for carbon analysis were collected on an impactor stage with lower and upper $D_{50 \text {,aero }}$ of 0.18 and $1.1 \mu \mathrm{m}$ over times of about 12 to $24 \mathrm{~h}$. Measurements at KCO described above were collected over a $24 \mathrm{~h}$ period by UMiami and $48 \mathrm{~h}$ period for the KCO-Caltech samples. The impactor stages chosen for the comparison were those that most closely approximated a $1.0 \mu \mathrm{m}$ size cut at $55 \% \mathrm{RH}$. Stacked filter units with a $D_{50 \text {,aero }}$ of approximately $1.3 \mu \mathrm{m}$ were used on 
Table A1. Samplers Used for Collecting Aerosol for Chemical Analysis

\begin{tabular}{|c|c|c|c|c|}
\hline Platform & Sampler & $D_{50, \text { aer } 0}, \mu \mathrm{m}$ & $\% \mathrm{RH}$ & Comments \\
\hline KCO-UMiami & $\begin{array}{l}\text { Sierra } \\
\text { Impactor }\end{array}$ & 1.25 & Ambient $^{\mathrm{a}}$ & $\begin{array}{c}D_{50, \text { aero }} \sim 1.1 \mu \mathrm{m} \\
\quad \text { at } 55 \% \mathrm{RH}^{\mathrm{b}}\end{array}$ \\
\hline KCO-Caltech & MOUDI & 1.8 & Ambient $^{\mathrm{a}}$ & $\begin{array}{l}D_{50, \text { aero }} \sim 1.6 \mu \mathrm{m} \\
\quad \text { at } 55 \% \mathrm{RH}^{\mathrm{b}}\end{array}$ \\
\hline C-130 & $\begin{array}{l}\text { Stacked Filter } \\
\text { Unit }\end{array}$ & 1.3 & $60-87$ & $\begin{array}{l}D_{50, \text { aero }} \sim 1.1 \text { to } \\
1.3 \mu \mathrm{m} \\
\text { at } 55 \% \mathrm{RH}^{\mathrm{b}}\end{array}$ \\
\hline RB & $\begin{array}{l}\text { Berner } \\
\text { Impactor }\end{array}$ & 1.1 & $55 \pm 5$ & \\
\hline
\end{tabular}

\footnotetext{
${ }^{\mathrm{a}} 80$ to $87 \% \mathrm{RH}$.

${ }^{\mathrm{b}} D_{50 \text {,aero }}$ conversion based on densities and hygroscpic growth calculated from mean KCO-Caltech composition.
}

the C-130. The stacked filter units were operated between 60 and $87 \%$ RH. Only low altitude samples $(<300 \mathrm{~m})$ from the $\mathrm{C}-130$ are considered for chemical comparisons.

\section{B.1.1.2. Sample Analysis}

[95] $\mathrm{RB}$ samples were analyzed for $\mathrm{SO}_{4}^{2-}, \mathrm{Na}^{+}$, and $\mathrm{K}^{+}$ using ion chromatography [Quinn et al., 1998]. The nonsea salt correction for $\mathrm{SO}_{4}^{2-}$ and $\mathrm{K}^{+}$was based on $\mathrm{Na}^{+}$. The KCO-UMiami samples were analyzed for $\mathrm{SO}_{4}^{2-}$ using ion chromatography and $\mathrm{Na}^{+}$using flame atomic absorption (D. Savoie, personal communication, 2000). KCO-Caltech samples were analyzed for $\mathrm{SO}_{4}^{2-}$ using ion chromatography [Mulik et al., 1976; Hughes et al., 1999]. The C-130 samples were analyzed for $\mathrm{SO}_{4}^{2-}, \mathrm{Na}^{+}$, and $\mathrm{K}^{+}$using ion chromatography [Gabriel et al., 2002]. Organic and elemental carbon from $\mathrm{KCO}$, the $\mathrm{RB}$, and the $\mathrm{C}-130$ were all determined using variations of thermal desorption/evolved gas analysis. As explained below, the analytical procedures are quite different, possibly giving disparate results for identical samples. In addition, sampling with quartz filters is subject to both positive and negative artifacts [Huebert and Charlson, 2000].

[96] OC and BC concentrations for samples collected on the RB were determined by a thermal desorption method using a commercial system (5500 c-mat, Ströhlein, Kaarst, Germany) [Neusüß et al., 2000, 2002]. The sample was placed in a quartz tube and heated to $500 \mathrm{C}$ under nitrogen to separate organic and elemental carbon. The carbon compounds that evaporated under these conditions were referred to as volatile carbon (OC). In a second step, the sample was heated under oxygen to $650^{\circ} \mathrm{C}$, where all carbon except carbonate is oxidized. This carbon mass was referred to as nonvolatile carbon (BC). The accuracy of the total carbon, TC, concentration is $\pm 20 \%$ not including artifact effects. Because there was no correction for charring of $\mathrm{OC}$ to $\mathrm{BC}$ during the analysis, the $\mathrm{BC}$ concentrations represent an upper limit.

[97] Samples collected at KCO by Caltech were analyzed for elemental and organic carbon content using the thermaloptical carbon analysis method of Huntzicker et al. [1982] as modified by Birch and Cary [1996]. A commercial instrument was used (Sunset Laboratory, OCEC analyzer, Portland, OR). The sample was placed in a quartz oven and heated to $870^{\circ} \mathrm{C}$ in a helium atmosphere to desorb organic compounds. After the oven was cooled to $600^{\circ} \mathrm{C}$, the temperature was increased a second time in a helium/oxygen atmosphere to oxidize elemental carbon. Correction for pyrolytic formation of elemental carbon during OC determination was accomplished using the methodology described in the paper by Kleeman et al. [1999]. The OC/ $\mathrm{BC}$ split (and optical correction) was performed on simultaneously sampled quartz filters and then applied to the MOUDI foil samples.

[98] Another evolved gas analysis (EGA) method [Mayol-Bracero et al., 2002] was used to determine the $\mathrm{OC}$ and $\mathrm{BC}$ concentrations in the $\mathrm{C}-130$ samples. A portion of the filter was heated at $12.5^{\circ} \mathrm{C} \min ^{-1}$ in an oxygen atmosphere from $50^{\circ} \mathrm{C}$ to $600^{\circ} \mathrm{C}$. A plot of the rate of carbon evolution versus temperature constitutes a thermogram. The area under the thermogram is equal to the total carbon content of the sample. As with the RB samples, the $\mathrm{BC}$ concentrations may represent an upper limit since there was no correction for charring of $\mathrm{OC}$ to $\mathrm{BC}$ during the analysis. This method is quantitative for $\mathrm{TC}$ within about $10 \%$ with a reproducibility of 3 to $5 \%$ not including artifact effects. Total aerosol mass was determined by gravimetric analysis for the RB samples. Substrates were weighed before and after sample collection at $33 \pm 3 \% \mathrm{RH}$ [Quinn et al., 1998]. Gravimetric analysis at $39 \pm 3 \% \mathrm{RH}$ was used to determine aerosol mass for the KCO-Caltech samples [Hughes et al., 1999]. Total mass for the C-130 samples was determined by summing the concentrations of the chemically analyzed species (ions, $\mathrm{BC}$, and $\mathrm{OC}$ converted to particulate matter using a factor of $1.5 \mu \mathrm{g}$ particular organic material (POM) mass per $\mu \mathrm{g}$ of $\mathrm{C}$ mass). Total mass for the KCO-UMiami samples is a sum of the ionic and ash concentrations. Summing chemically analyzed species to estimate total aerosol mass may underdetermine the mass if significant species on a mass-basis are not analyzed (including water). Other chemical species were not compared such as iron (which was not analyzed on all platforms) and nitrate which can suffer from sampling artifacts.

\section{B.2. Microphysical Characteristics}

[99] Ambient aerosol sizes measured during INDOEX range from a few nanometers to hundreds of micrometers. However, the size range most likely to dominate aerosol light scattering and extinction in the atmosphere is generally from about 0.1 to $10 \mu \mathrm{m}$ diameter, $\mathrm{Dp}$, and includes most aerosol mass. Because the scattering per unit mass (or mass scattering efficiency, MSE) is a strong function of size with a maximum around $0.5 \mu \mathrm{m}$ (for wavelengths of $550 \mathrm{~nm}$ ) it is essential to measure and model the size distribution accurately in order to provide a foundation for interpreting optical effects in terms of constituent masses and to reduce uncertainties in "closure" experiments. Various techniques used to size aerosol based upon different physical principles are listed in Table 1. These include optical techniques based upon scattered light such as a custom Laser Optical Particle Counter (OPC), a forward Scattering Spectrometer Probe (FSSP-300) and a Passive Cavity Aerosol Spectrometer Probe (PCASP), all obtained from Particle Measurement Systems, Boulder, CO. Mobility techniques based upon diffusion of charged aerosol in an electric field were used in various Differential Mobility Analyzer (DMA) or Tandem Radial DMA systems. Other distributions were obtained by impaction based upon the aerodynamic diameters resulting from inertial and drag forces acting on a particle (MOUDI) and also scanning electron microscopy 
(SEM) based upon the projected area of a particle collected on a substrate (not discussed here).

\section{B.2.1. KCO Aerosol Size Distributions and Concentrations}

[100] Size distributions were measured at KCO over limited periods and included aerodynamic size cuts with relatively large bins afforded by a MOUDI [Marple et al., 1991] and sizing from a DMA $(20<\mathrm{Dp}<530 \mathrm{~nm}$; Cantrell et al., submitted manuscript, 2000). Four 10-stage MOUDIs were simultaneously operated to measure $46 \mathrm{~h}$ average fine particulate mass concentration and chemical composition as a function of particle size. We note that the near ambient $\mathrm{RH}$ $(80-87 \%)$ used for this system is much higher than most other INDOEX sample systems so that chemical species present in soluble aerosol will be collected in larger size bins than other samplers. Unfortunately, comparable sampling instrumentation was not operating during limited sideby-side comparisons and will not be directly compared here.

\section{B.2.2. RB Aerosol Size Distributions}

\section{and Concentrations}

[101] Total particle number concentrations were measured with TSI $3010(10 \mathrm{~nm}<\mathrm{Dp}<3000 \mathrm{~nm})$ and TSI $3025(3$ $\mathrm{nm}<\mathrm{Dp}<3000 \mathrm{~nm}$ ) condensation particle counters (CPCs) operated directly off one of the $1.9 \mathrm{~cm}$ sampling tubes. One tube supplied air to a differential mobility particle sizer (DMPS) located inside the humidity-controlled box at the base of the mast. The DMPS (Heuke-type, medium length) was connected to a TSI 3010 particle counter with an aerosol flow rate of $0.5 \mathrm{~L} \mathrm{~min}^{-1}$ and a sheath airflow rate of $5 \mathrm{~L} \mathrm{~min}^{-1}$ humidified to $55 \% \mathrm{RH}$. Data were collected in 27 size bins with midpoints ranging from 22 to $900 \mathrm{~nm}$ diameter. Another tube supplied air to an identical DMPS that measured under "dry" conditions $(\mathrm{RH}<10 \%)$. The DMPS data were filtered to eliminate periods of calibration and instrument malfunction and periods of ship contamination (based on relative wind and high and rapid changes in $\mathrm{CN}$ counts). The filtered mobility distributions were then converted to number-size distributions using the inversion routine of Stratman and Wiedensohler [1997]. The data were corrected for diffusional losses [Covert et al., 1997] and size dependent counting efficiencies [Wiedensohler et al., 1997] based on earlier calibration exercises.

[102] The accuracy of both the particle sizing and the number of particles in each size bin depends on the stability of the flow rates [Bates et al., 2002]. Three of the four DMPS flows (CPC, Sheath and Excess) were controlled independently in these three systems. Drift in the CPC, sheath, and excess flows was generally less than one percent during the cruise (mean $0.63 \pm 0.55 \%$ ) which translates into a particle sizing error of about 1 percent. However, a relative drift of $1 \%$ in the sheath to excess flow implies a $10 \%$ change in inlet flow and thus a $10 \%$ change in the number concentration. A change in the inlet flow also changes the transfer function of the DMA which compounds this error, e.g., for this case the combined error is on the order of $15 \%$. During INDOEX, the average integrated RBDMPS number concentration operated at $55 \% \mathrm{RH}$ averaged $6.5 \pm 7.1 \%$ lower than the total number measured by the CPC (TSI 3010). The integrated number concentration from the DMPS system operated at 10\% RH averaged $19 \pm 9.3 \%$ lower than the total number measured by the CPC. To correct for the number concentration error, the DMPS data reported here have been normalized using the $30 \mathrm{~min}$ average ratio of the total CPC number concentration to the integrated DMPS number concentration. (Note: the DMPS data collected at 55\% RH have been used in this paper for the regional average size distributions).

[103] Unfortunately, this instrument was not working during the C-130 flyby which necessitated using the "dry" DMPS data collected at 10\% RH for comparison with the C-130 size distribution data.) During the C-130 flyby (discussed above) the integrated number concentration from this DMPS system operated at $10 \% \mathrm{RH}$ and was $27 \%$ lower than the total number measured by the CPC. The DMPS size-distribution data used in the flyby comparison were therefore uniformly increased by this factor. The data were then converted from $10 \% \mathrm{RH}$ to $55 \% \mathrm{RH}$ using the densities and associated water masses calculated with a thermodynamic equilibrium model (AeRho) using the measured chemical data [Quinn et al., 2000] for comparison to the $\mathrm{C}-130$ data. (Note: the $\mathrm{C}-130$ data used in the comparison were adjusted to $55 \% \mathrm{RH}$ from measured DMA RH using the growth curves shown in Figure 2).

[104] Another tube supplied an Aerodynamic Particle Sizer (APS-TSI 3320) in the humidity-controlled box at the base of the mast. The APS measured the number size distribution between 0.6 and $9.6 \mu \mathrm{m}$ aerodynamic diameter. Based on comparisons between gravimetrically determined mass at $33 \%$ RH and mass derived from the APS, it was assumed that heating in the APS dried the coarse mode aerosol to below its efflorescence point. Therefore, the APS data shown here were converted from aerodynamic diameters at a measurement RH (assumed below the efflorescence point of sea salt) to geometric diameters at $55 \% \mathrm{RH}$ using calculated densities and the water masses associated with the inorganic ions at 33 and $55 \% \mathrm{RH}$. The densities and associated water masses were calculated with AeRho using the measured chemical data [Quinn et al., 2000]. However, we recognize that sea-salt mixed with other aerosol species may result in efflorescence behavior that differs from pure sea-salt.

\section{B.2.3. C-130 Aerosol Size Distributions and Concentrations}

[105] Size distributions aboard the C-130 were obtained through a combination of DMA and OPC inside the aircraft and FSSP and PCASP probes exposed to ambient conditions on the wing. Both Radial DMA [Zhang et al., 1995] and OPC measurements included upstream controlled heaters that were used to assess aerosol volatility at 150 and $380^{\circ} \mathrm{C}$ [Clarke, 1991; Clarke et al., 1999]. The DMA was operated with dessicated $(\mathrm{RH}<10 \%)$ sheath airflow of $5 \mathrm{~L}$ $\mathrm{min}^{-1}$ and a sample flow of $0.7 \mathrm{~L} \mathrm{~min}-1$ with the latter showing 1 to $5 \%$ variation during 7 mission calibrations. $\mathrm{RH}$ was measured at the exit of the instrument and was generally around $20 \%$ for the near surface data presented here. Sheath air was recirculated with a sealed pump system that ensured sheath and excess flows were identical and that sample flow and detector flows were identical. In order to permit rapid scan times for the DMA (about 90 s) necessary for aircraft sampling, the upper size limit was set to 0.20 $\mu \mathrm{m}$. This also allowed overlap with the OPC that sized from 0.15 to $7 \mu \mathrm{m}$. OPC and DMA distributions were combined about the common point of $0.2 \mu \mathrm{m}$ where agreement in concentration was generally within the $5-10 \%$ combined 
flow uncertainty evident from flow calibrations. A $1 \mu \mathrm{m}$ impactor upstream of the DMA was used to eliminate larger aerosol.

[106] In order to minimize size fluctuations due to humidity changes the OPC was operated with a sample flow diluted with equal parts of dry air to result in an instrument RH of generally $30-40 \%$ at low altitudes. Size dependent corrections to OPC data also had to be made due to losses of super-micrometer aerosol in the community aerosol inlet (CAI) mounted on the C-130. This had negligible impact on aerosol total number but results from the PELTI experiment [Huebert et al., 2000] showed about a factor of 2 or more underestimate of particles larger than about $3 \mu \mathrm{m}$. A secondary dependence was also found on CAI losses depending upon whether particles were "wet" or "dry." Additional losses, particularly for large particles, are also expected within the tubing leading to the OPC. These losses have not been well characterized so no corrections can be made. In addition to onboard aerosol measurements the $\mathrm{C}$ 130 also included the wing-mounted Forward Scattering Spectrometer Probes (FSSP) and Passive Cavity Aerosol Spectrometer Probe (PCASP). These were intended to provide measurements of ambient aerosol size and to capture sizes larger than those expected to be aspirated in to the $\mathrm{C}-130$. These probes offer the advantage of sampling particles in-situ but have limitations due to uncertainties in refractive index and aspects of instrument operation.

[107] CPCs (TSI 3760 and 3025) were also operated at cabin temperatures and at $380^{\circ} \mathrm{C}$ to measure total and refractory aerosol number. In this way total number integrated over thermally resolved DMA/OPC size distributions could be compared to measured number as a test of data consistency. The low altitude C-130 measurements found the 3010 and 3025 concentrations differed by much less than the combined absolute flow uncertainty of about $7 \%$ and therefore are indicative of few particles present below $15 \mathrm{~nm}$. Routine in-flight filter tests were used to confirm proper zero counts in all instruments and CPCs were tested on the ground for flow and count performance. Comparison of integral DMA/OPC data with 3010 data typically differed by less than $15 \%$ but was generally lower on the average suggesting some small particle losses in the DMA system. Unlike the RB data, the C-130 DMA/OPC data did not have to be rescaled here to agree with CPC counts.

\section{B.2.4. Sizing Uncertainty Issues}

[108] In order to interpret measurements in terms of geometric physical size, as is generally needed for model applications, all of these approaches involve some assessment or assumption of aerosol properties such as refractive index, density, water uptake, shape etc. As a result, not only measurement uncertainties (e.g., flow rates, inlet efficiencies, etc.) influence sizes and concentrations but also uncertainties in these aerosol properties. Hence, appropriate geometric size distributions are obtained from measured data only after corrections (D/Do growth versus RH, density, refractive index) consistent with the other chemical and optical measurements made during INDOEX.

[109] Aerosol growth in response to increasing $\mathrm{RH}$ is largely determined by the role of hygroscopic materials such as deliquescent salts (e.g., ammonium sulfate) [Tang and Munkelwitz, 1977]. Knowledge of the hygroscopic constituents can be used with "mixing rules" for molar composi- tion to predict aerosol growth in response to increasing $\mathrm{RH}$ [Stelson, 1990]. Alternatively, empirical growth curves can be established for aerosol size classes by measuring the size dependent growth such as those carried out during INDOEX aboard the RB (Figure 2). With this information, size distributions measured at a given instrumental $\mathrm{RH}$ were adjusted for another $\mathrm{RH}$, for comparison to data from other instruments or other conditions (e.g., ambient).

\section{B.3. Optical Characteristics}

[110] The aerosol optical properties considered here are the ones most important to aerosol forcing of climate: light scattering coefficient $\left(\sigma_{s p}\right)$, light absorption coefficient $\left(\sigma_{a p}\right)$, single-scattering albedo $\left(\omega_{\mathrm{o}}\right)$, hemispheric backscatter fraction (b) and the Angström exponent (å). Most or all of these were measured on the major platforms participating in INDOEX. Some platforms included additional parameters, such as the fractions of light scattering and absorption attributable to submicrometer particles $\left(\mathrm{R}_{s p}, \mathrm{R}_{a p}\right)$, the dependence of aerosol light scattering on relative humidity $(\mathrm{f}(\mathrm{RH}))$, and the wavelength-dependence of aerosol light scattering (e.g., the Ångström exponent å $\left(\lambda_{1}, \lambda_{2}\right)=$ $\left(\log \left(\sigma_{s p, 1} / \sigma_{s p, 2}\right) / \log \left(\sigma_{2} / \sigma_{1}\right)\right.$. Aerosol light scattering coefficient was measured with a multiwavelength instrument equipped with a backscatter shutter (Model 3563, TSI, Inc., St. Paul, Minnesota) or single-wavelength instrument (Model M902, Radiance Research, Seattle, Washington). The TSI nephelometer operates at wavelengths of 450, 550, and $700 \mathrm{~nm}$, over an angular range of 7-170 degrees (total scatter) and 90-170 degrees (hemispheric backscatter). The Radiance nephelometer operates at a wavelength of $530 \mathrm{~nm}$, over an angular range of 9-168 degrees. Both nephelometers were corrected for truncation effects over the appropriate angular range. Aerosol light absorption coefficient was measured with a continuous light absorption photometer (Model PSAP, Radiance Research, Seattle, Washington). All results were corrected using the scheme reported by Bond et al. [1999] for sample area, flowrate, and nonidealities in the manufacturer's calibration based on a calibration of the instrument at $550 \mathrm{~nm}$. Hence, all absorption data are reported at $550 \mathrm{~nm}$.

[111] Aerosol extensive (dependent upon the aerosol concentration, e.g., $\sigma_{a p}, \sigma_{s p}$ ) and intensive (independent of the amount of aerosol present, e.g., $\varpi_{\mathrm{o}}, \mathrm{b}$, ̊̊) optical properties measured by the various platforms deployed during INDOEX were compared for various cases. In order to perform the interplatform comparisons, the measurements were adjusted so that both platforms were on the same relative basis: $\mathrm{RH}$, wavelength, and standard temperature (20C) and pressure. RH adjustments were made based on fitting the power law equation: $\mathrm{f}(\mathrm{RH})=\mathrm{a}(1-(\mathrm{RH} / 100))^{-\mathrm{b}}$ to $\mathrm{KCO}$ humidigraph measurements (Figure 1). Wavelength adjustments were made assuming the submicrometer Angström exponent was constant over the wavelength range of

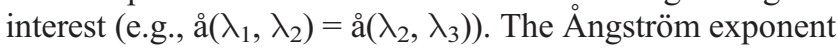
is typically used as an indicator of the presence (or absence) of super-micrometer particles [Reid et al., 1998]. For these measurements where the aerosol is predominantly in the submicrometer fraction there is little difference between sub and total Ångström exponent. However, even when the super-micrometer fraction is significant, shifts in the sub- 
Table A2. Estimated Uncertainties in $\sigma_{s p}{ }^{\mathrm{a}}$

\begin{tabular}{lcccccc}
\hline Bsp & Noise & Drift & Calibration & Truncation & Stp & Total \\
\hline 1 & 1.25 & 0.44 & 0.08 & 0.02 & 0.003 & 1.33 \\
10 & 1.56 & 0.80 & 0.75 & 0.22 & 0.03 & 1.92 \\
20 & 1.84 & 1.20 & 1.50 & 0.44 & 0.07 & 2.70 \\
50 & 2.50 & 2.40 & 3.75 & 1.10 & 0.17 & 5.23 \\
100 & 3.32 & 4.40 & 7.51 & 2.20 & 0.34 & 9.58 \\
\hline
\end{tabular}

${ }^{\mathrm{a}}$ At $550 \mathrm{~nm}$ for $1 \mathrm{~min}$ averaging time and submicrometer particles (in $\left.\mathrm{Mm}^{-1}\right)$.

micrometer Ångström exponent can provide qualitative information about shifts in the aerosol size distribution, although the relationship between aerosol size and submicrometer Ångström exponent is not as strong as it is when super-micrometer particles are included. Unless noted otherwise, measurements were adjusted to $55 \%$ relative humidity and $550 \mathrm{~nm}$ wavelength and the aerodynamic $\mathrm{Dp}<1 \mu \mathrm{m}$ $[\mathrm{Dp}<0.75 \mu \mathrm{m}$ geometric diameter] was used as the size cut. No relative humidity adjustments were attempted for light absorption measurements.

\section{B.3.1. Instrument Uncertainties: Nephelometer}

[112] Calculation of the measurements uncertainty of each nephelometer followed the protocol of Anderson et al. [1999]. The measurement uncertainty associated with the TSI 3563 nephelometer was calculated from five known sources and is expressed as a linear combination of the following terms.

$$
\delta \sigma_{\text {total }}^{2}=\delta \sigma_{\text {noise }}^{2}+\delta \sigma_{\text {drift }}^{2}+\delta \sigma_{\text {cal }}^{2}+\delta \sigma_{\text {trunc }}^{2}+\delta \sigma_{\text {stp }}^{2}
$$

Here $\delta \sigma_{p}$ designates the uncertainty in $\sigma_{p}$ associated with the parameter, $p$. These arise from (1) noise in the filtered air scattering coefficient, (2) drift in the calibration, (3) uncertainty in the instrument calibration to Rayleigh scattering of dry air and $\mathrm{CO}_{2}$, (4) the truncation of near forward scattered light, and (5) uncertainty in instrument pressure and temperature in conversion of data to STP. The associated uncertainties for each parameter for 1-min averages are listed in Table A2 with varying magnitude of the scattering coefficient.

[113] Uncertainty associated with differences in the aerosol inlets and tubing is expected to be insignificant for submicrometer aerosol. Losses within the nephelometer itself have been found to be negligible for submicrometer particles and $5-10 \%$ for super micrometer particles [Anderson and Ogren, 1998]. The variation in particle size with relative humidity and hence the particle transmission through a submicrometer impactor operating upstream of the nephelometer will vary with the particle type. For relative humidities below $50 \%$ we estimate this uncertainty to be less than $5 \%$ based on Berner impactor efficiency curves and estimates of the scattering size distribution. In addition to $\mathrm{RH}$, flowrate affects the $50 \%$ aerodynamic cut off diameter of the impactor. Running the Berner type impactors at a flow $10 \%$ lower than $30 \mathrm{lpm}$ yields a $5 \%$ change in cut size. Typically flows for all 4 systems were within $1-2 \%$ of expected flow rate.

[114] For low scattering values instrument noise is the prevalent source of uncertainty while for higher scattering coefficients both noise and instrument truncation uncertain- ties dominate. Uncertainty for low signal values can be greatly reduced by increasing the signal averaging time. For a 10-min averaging time the uncertainty associated with noise for a $\sigma_{s p}$ of $1 \mathrm{Mm}^{-1}$ is $0.40 \mathrm{Mm}^{-1}$. For the Radiance Research nephelometer used by UMiami the overall uncertainty for submicrometer scattering was calculated to be $8 \%$ at $50 \mathrm{Mm}^{-1}$ equivalent to an uncertainty of $4 \mathrm{Mm}^{-1}$. This value was calculated considering noise, calibration uncertainties [e.g., calibration gas impurities, STP correction), angular nonidealities (e.g., truncation), adjustment to 550 $\mathrm{nm}$ wavelength and adjustment to $55 \% \mathrm{RH}$ ( $\mathrm{H}$. Maring, personal communication, 2000).

\section{B.3.2. Instrument Uncertainties: PSAP}

[115] Uncertainties in the PSAP have been described by Bond et al. [1999] and the appropriate corrections have been applied to PSAP measurements made at INDOEX. Corrections have been made for spot size, flow rate, interpretation of scattering as absorption and instrument response to absorption. Bond et al. also found instrumental variability to be $6 \%$ of the measured absorption, hence for the average absorption coefficient measured at INDOEX instrumental variability is $1 \mathrm{Mm}^{-1}$. Instrument noise (i.e., detection limit - a signal/noise ratio of unity), determined by measuring particle-free air, is $0.1 \mathrm{Mm}^{-1}$ for hourly averaged data and $0.9 \mathrm{Mm}^{-1}$ for minute averaged data. Absorption coefficients measured during INDOEX were much higher than the detection limit.

[116] Acknowledgments. This research was funded in part by the National Science Foundation [ATM-9612891 and the NASA Global Aerosol Climatology Project NAG-5-8118 (A.D.C., S.H.); ATM-ATM9612888 J.A.O., E.A., and A.J.] and the Aerosol Program of the NOAA Climate and Global Change Program and the NASA Global Aerosol Climatology Project (P.K.Q. and T.S.B.). We would also like to thank the following people for contributing their data to this effort: M. O. Andreae, J. Prospero, D. Baumgardner, A. Wiedensholer, A. Novak, D. Covert, L. Hughes, R. Gabriel, T. Kirchstetter, C. Neusüß, T. Novakov, A. Jayaraman, G. Shaw, and W. Cantrell. SOEST contribution 6022 .

\section{References}

Anderson, T. L., and J. A. Ogren, Determining aerosol radiative properties using the TSI 3563 integrating nephelometer, Aerosol Sci. Technol., 29, $57-69,1998$.

Anderson, T. L., D. S. Covert, J. D. Wheeler, J. M. Harris, K. D. Perry, B. E. Trost, D. J. Jaffe, and J. A. Ogren, Aerosol back scatter fraction and single scattering albedo: Measured values and uncertainties at a coastal station in the Pacific Northwest, J. Geophys. Res., 104, 26,793-26,807, 1999.

Bates, T. S., D. J. Coffman, D. S. Covert, and P. K. Quinn, Regional marine boundary layer aerosol size distributions in the Indian, Atlantic and $\mathrm{Pa}-$ cific Oceans: A comparison of INDOEX measurements with ACE-1, ACE-2, and aeroso199, J. Geophys. Res., 107, doi:10.1029/ 2001JD001174, in press, 2002.

Baumgardner, D., J. E. Dye, R. G. Knollenberg, and B. W. Gandrud, Interpretation of measurements made by the FSSP-300X during the Airborne Arctic Stratospheric Expedition, J. Geophys. Res., 97, 8035-8046, 1992.

Birch, M. E., and R. A. Cary, Elemental carbon-based method for monitoring occupational exposures to particulate diesel exhaust, Aerosol Sci. Technol., 25, 221-241, 1996.

Blomquist, B. W., B. J. Huebert, S. G. Howell, M. Litchy, C. H. Twohy, A. Schanot, D. Baumgardner, B. Lafleur, R. Seebaugh, and M. L. Laucks, An evaluation of the community aerosol inlet for the NCAR C-130 research aircraft, J. Atmos. Oceanic Technol., 18, 1387-1397, 2001.

Bond, T. C., T. L. Anderson, and D. Campbell, Calibration and intercomparison of filter-based measurements of visible light absorption by aerosols, Aerosol Sci. Technol., 30, 582-600, 1999.

Cachier, H., C. Liousse, P. Buat-Menard, and A. Gaudichet, Particulate content of savanna fire emissions, J. Atmos. Chem., 22, 123-148, 1995. Clarke, A. D., "A thermo-optic technique for in-situ analysis of size-re- 
solved aerosol physicochemistry", Atmos. Environ. Part A, 25(3/4), 635-644, 1991

Clarke, A. D., F. Eisele, V. N. Kapustin, K. Moore, R. Tanner, L. Mauldin, M. Litchy, B. Lienert, M. A. Carroll, and G. Albercook, Nucleation in the equatorial free troposphere: Favorable environments during PEM-Tropics, J. Geophys. Res., 104, 5735-5744, 1999.

Covert, D. S., J. L. Gras, A. Weidensohler, and F. Stratmann, Comparison of directly measured $\mathrm{CCN}$ with $\mathrm{CCN}$ modeled from the number-size distribution in the marine boundary layer during ACE-1 at Cape Grim, Tasmania, J. Geophys. Res., 103, 16,597-16,608, 1998.

Delene, J., and A. Ogren, Variability of aerosol optical properties at four North American surface monitoring sites, J. Atmos. Sci., in press, 2001.

Ferek, R. J., J. S. Reid, P. V. Hobbs, D. R. Blake, and C. Liousse, Emission factors of hydrocarbons, halocarbons, trace gases, and particles from biomass burning in Brazil, J. Geophys. Res., 103, 32,107-32,118, 1998.

Gabriel, R., O. L. Mayol-Bracero, and M. O. Andreae, Chemical characterization of submicron aerosol particles collected over the Indian Ocean, $J$. Geophys. Res., 107, doi:10.1029/2000JD000034, in press, 2002.

Garvey, D. M., and R. G. Pinnick, Response characteristics of particle measurement systems active scattering aerosol spectrometer probe (ASASP-X), Aerosol Sci. Technol., 2, 477-488, 1983.

Huebert, B. J., and R. J. Charlson, Uncertainties in data on organic aerosols, Tellus, Ser. B, 52, 1249-1255, 2000.

Huebert, B. J., D. Covert, A. Clarke, S. Howell, J. Anderson, and C. Wilson, Airborne performance tests of a new low-turbulence inlet (LTI) for aerosols, presented at Am. Assoc. for Aerosol Res. Meeting, Cincinnatti, Ohio, 2000.

Hughes, L., The size and composition distribution of atmospheric particles in southern California, Environ. Sci. Technol., 33, 3506-3515, 1999.

Huntzicker, J. J., R. L. Johnson, J. J. Shah, and R. A. Cary, Particulate Carbon, Atmospheric Life Cycle, edited by G. T. Wolff and R. L. Klimisch, pp. 79-88, Plenum, New York, 1982.

Jayaraman, A., S. K. Satheesh, A. P. Mitra, and V. Ramanathan, Latitude gradient in aerosol properties across the Inter Tropical Convergence Zone: Results from the joint Indo-US study onboard Sagar Kanya, Curr. Sci., 80, 128-137, 2001.

Kleeman, M. J., J. J. Schauer, and G. R. Cass, Size and composition distribution of fine particulate matter emitted from wood burning, meat charbroiling, and cigarettes, Environ. Sci. Technol., 33, 3516-3523, 1999.

Kotchenruther, R. A., P. V. Hobbs, and D. A. Hegg, Humidification factors for atmospheric aerosols off the mid-Atlantic coast of the United States, J. Geophys. Res., 104, 2239-2252, 1999.

Liu, B. Y. H., D. Y. H. Pui, X. Q. Wang, and C. W. Lewis, Sampling of carbon fiber aerosols, Aerosol Sci. Technol., 2, 499-511, 1983.

Marple, V. A., K. L. Rubow, and S. M. Behm, A micro-orifice uniform deposit impactor (MOUDI): Description, calibration and use, 14(4), 434-446, 1991

Masonis, S. J., K. Franke, A. Ansmann, D. Mueller, D. Althausen, J. A. Ogren, A. Jefferson, and P. J. Sheridan, An intercomparison of aerosol light extinction and $180^{\circ}$ backscatter as derived using in situ instruments and Raman lidar during the INDOEX field campaign, J. Geophys. Res., 107, doi:10.1029/2000JD000035, in press, 2002.

Mayol-Bracero, O. L., R. Gabriel, M. O. Andreae, T. W. Kirchstetter, T. Novakov, J. A. Ogren, P. J. Sheridan, and D. Streets, Carbonaceous aerosols over the Indian Ocean during INDOEX: Chemical characterization, optical properties, and probable sources, J. Geophys. Res., 107, doi:10.1029/2000JD000039, in press, 2002.

Mulik, J., R. Puckett, D. Williams, and E. Sawicki, Ion chromatographic analysis of sulfate and nitrate in ambient aerosols, Anal. Lett., 9, 653663, 1976.

Neusüß, C., D. Weise, W. Birmili, H. Wex, A. Wiedensohler, and D. S. Covert, Size-segregated chemical, gravimetric and number distribution- derived mass closure of the aerosol in Sagres, Portugal during ACE-2, Tellus, 52, 169-184, 2000.

Neusüß, C., T. Gnauk, A. Plewka, H. Herrmann, and P. K. Quinn, Carbonaceous aerosol over the Indian Ocean: OC/EC fractions and selected specifications from size-segregated onboard samples, J. Geophys. Res., 107, doi:10.1029/2001JD000327, in press, 2002.

Quinn, P. K., and D. J. Coffman, Local closure during ACE 1: Aerosol mass concentration and scattering and backscattering coefficients, J. Geophys. Res., 103, 16,575-16,596, 1998.

Quinn, P. K., D. J. Coffman, V. N. Kapustin, T. S. Bates, and D. S. Covert, Aerosol optical properties in the marine boundary layer during ACE 1 and the underlying chemical and physical aerosol properties, J. Geophys. Res., 103, 16,547-16,563, 1998 .

Quinn, P. K., et al., Surface submicron aerosol chemical composition: What fraction is not sulfate?, J. Geophys. Res., 105, 6785-6806, 2000.

Ramanathan, V., et al., The Indian Ocean Experiment: An integrated assessment of the climate forcing and effects of the great Indo-Asian haze, $J$. Geophys. Res., 106(22), doi:10.1029/2001JD900133, 2001.

Reid, J. S., P. V. Hobbs, R. J. Ferek, D. R. Blake, J. V. Martins, M. R. Dunlap, and C. Liousse, Physical, chemical and optical properties of regional hazes dominated by smoke in Brazil, J. Geophys. Res., 103, 32,059-32,080, 1998

Savoie, D. L, R. Arimoto, W. C. Keene, J. M. Prospero, R. A. Duce, and J. N. Galloway, Marine biogenic and anthropogenic contributions to nonsea-salt sulfate in the marine boundary layer over the North Atlantic Ocean, J. Geophys. Res., 107, 4356, doi:10.1029/2001JD000970, 2002.

Sheridan, P. J., A. Jefferson, and J. A. Ogren, Spatial variability of aerosol radiative properties over the Indian Ocean during INDOEX, J. Geophys. Res., 107, doi:10.1029/2000JD000166, in press, 2002.

Stelson, A. W., Urban aerosol refractive index prediction by partial molar fraction approach, Environ. Sci. Technol., 1676-1680, 1990.

Stratman, F., and A. Wiedensohler, A new data inversion algorithm for DMPS measurements, J. Aerosol Sci., 27, 339-340, 1997.

Swietlicki, E., et al., Hygroscopic properties of aerosol particles in the northeastern Atlantic during ACE-2, Tellus, Ser. B, 2, 201-227, 2000.

Tang, I. N., and H. R. Munkelwitz, Aerosol growth studies, III, Ammonium bisulfate aerosol in a moist atmosphere, J. Aerosol. Sci., 8, 321-330, 1977.

Turpin, B. J., P. Saxena, and E. Andrews, Measuring and simulating particulate organics in the atmosphere: Problems and prospects, Atmos. Environ., 34, 2983-3013, 2000

Weidensohler, A., et al., Intercomparison study of size dependent counting efficiency of 26 condensation particle counters, Aerosol Sci. Technol., 27, 224-242, 1997

Zhang, S. H., Y. Akutsu, L. M. Russell, R. C. Flagan, and J. H. Seinfeld, Radial differential mobility analyzer, Aerosol. Sci. Technol., 23, 357371,1995

E. Andrews, A. Jefferson, and J. A. Ogren, Climate Monitoring and Diagnostics Laboratory, National Oceanic and Atmospheric Administration, Boulder, CO, USA.

G. Cass, School of Earth and Atmospheric Sciences, Georgia Tech, Atlanta, GA, USA.

A. D. Clarke and S. Howell, University of Hawaii, Honolulu, HI 96822, USA. (tclarke@soest.hawaii.edu)

H. Maring and D. Savoie, RSMAS/MAC, University of Miami, Miami, FL, USA

A. Massling, Institute for Tropospheric Research, Leipzig, Germany. O. Mayol-Bracero, Max Planck Institute for Chemistry, Mainz, Germany. P. K. Quinn and T. S. Bates, Pacific Marine Environmental Laboratory, National Oceanic and Atmospheric Administration, Seattle, WA, USA. 


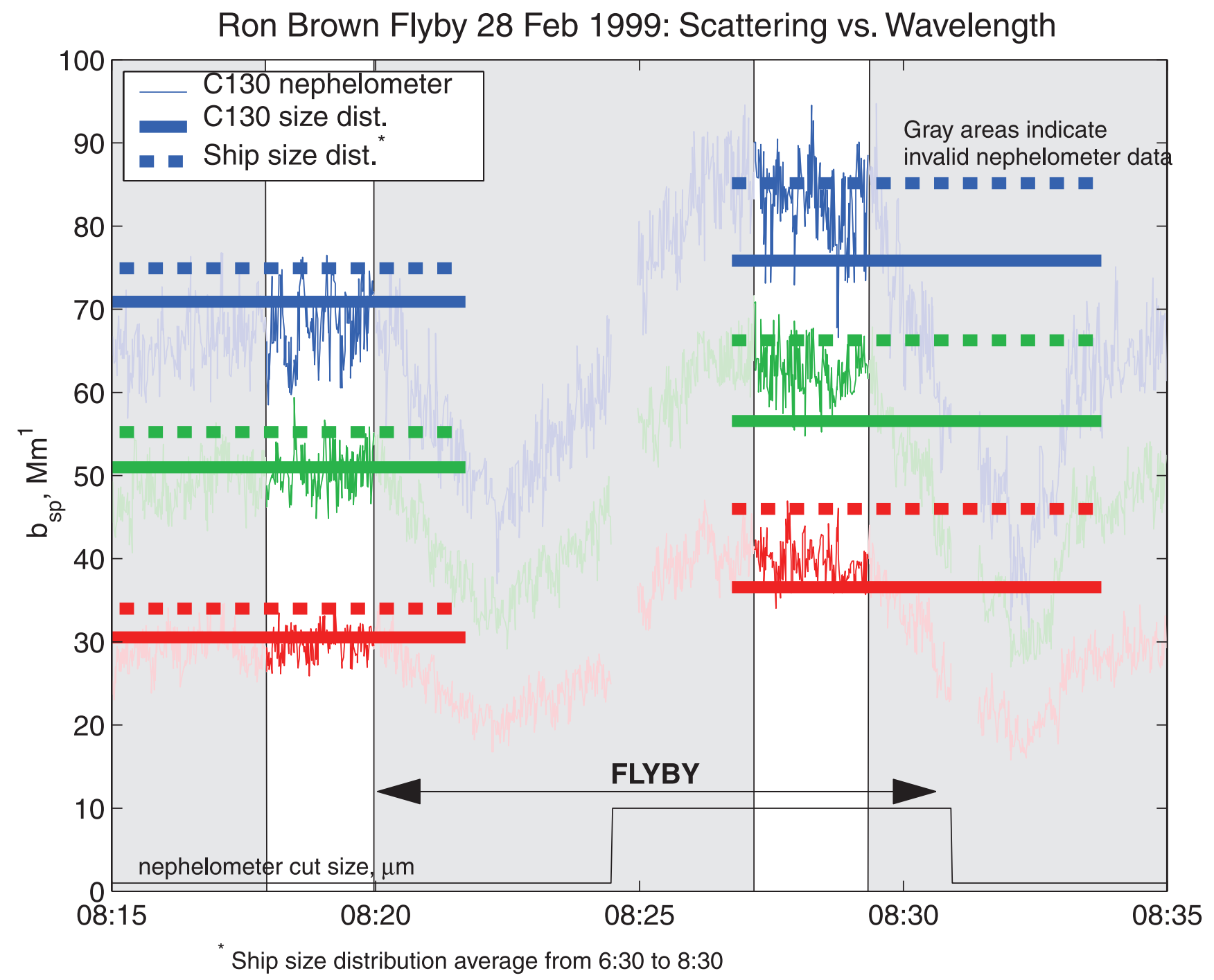

Figure 9. Light scattering values measured (C-130) and calculated from size distributions during RB flyby. The first time period is for submicrometer aerosol and the second is for total aerosol. 
MSE from C130 and R/V Ron Brown

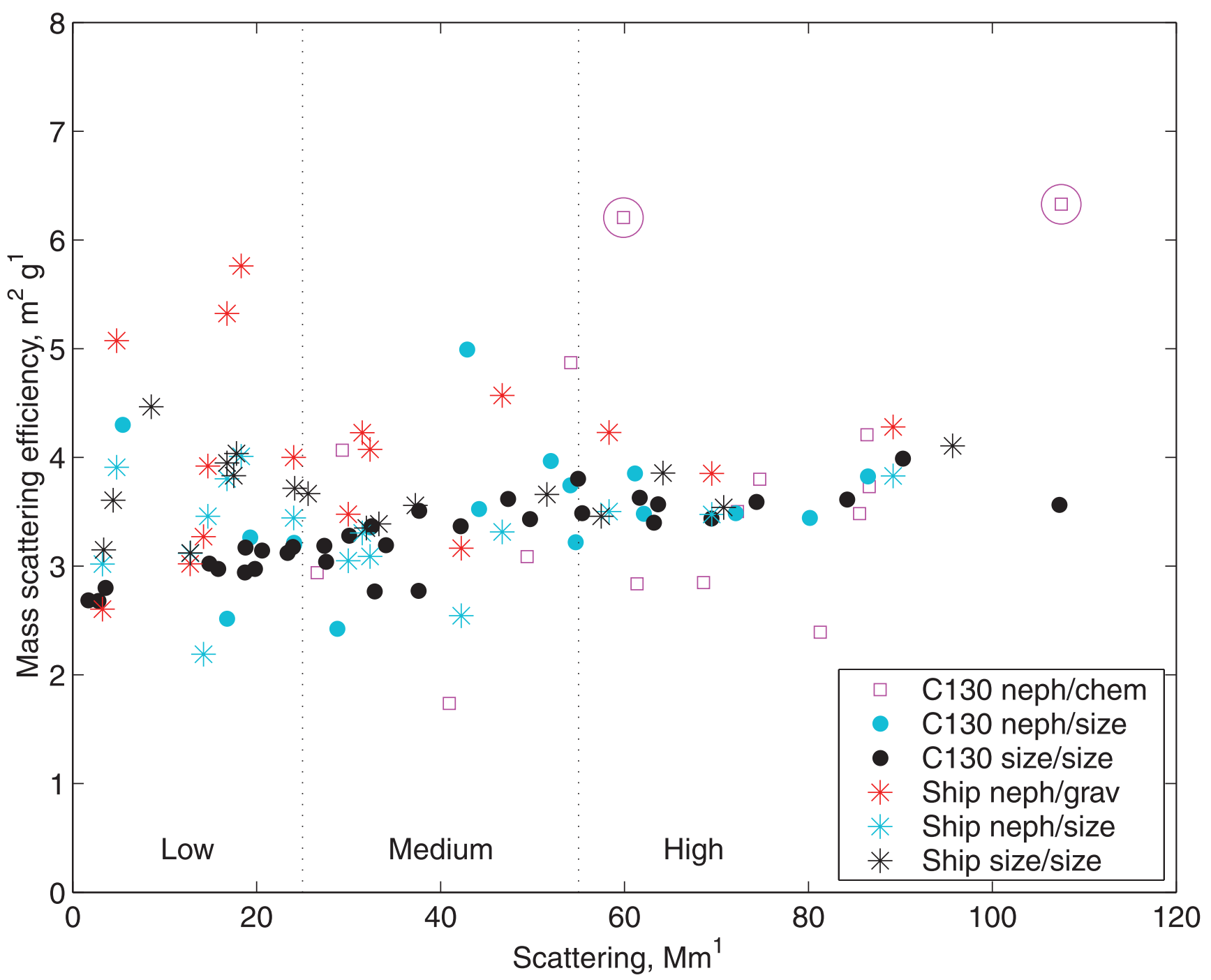

Figure 10. MSE values as a function of scattering values for various measurements (see text). 